Memória associativa em redes neurais realimentadas

Leonardo Garcia Corrêa 



\title{
Memória associativa em redes neurais realimentadas
}

\author{
LEONARDo GaRCIA CORRÊA
}

Orientador: Prof. Dr. Zhao Liang

Dissertação apresentada ao Instituto de Ciências Matemáticas e de Computação da Universidade de São Paulo, ICMC-USP, como parte dos requisitos para obtenção do título de Mestre em Ciências de Computação e Matemática Computacional.

\section{USP - São Carlos \\ maio/2004}

\footnotetext{
${ }^{1}$ Este trabalho teve suporte financeiro do $\mathrm{CNPq}$.
} 

Aos meus pais,

Valmir e Cida. 



\section{Agradecimentos}

Tive muitos "orientadores" nesse período de mestrado, e muitas foram as pessoas a quem gostaria de agradecer, entre elas:

Ao Zhao, pela oportunidade que me proporcionou de ingressar nesse programa de mestrado.

Ao Alexandre Delbem, que me orientou nesse último ano, mesmo não sendo oficialmente meu orientador do programa.

Ao Prof. Luís Alberto da Engenharia Elétrica, que sanou muitas das minhas dúvidas quanto à análise de sistemas não-lineares.

Ao José Paulo da Matemática, pelas discussões e provas de teoremas que muito me ajudaram.

Aos integrantes da 160, que representaram a minha família nesses últimos dois anos, e que me ajudaram muito, em todos os aspectos.

Aos meus pais, que possibilitaram que eu estivesse aqui, e também aos meus irmãos, Poli e Rafa.

À todas as amizades que fiz no mestrado, e que foram muitas, entre elas: Bruno, Waldo, Cláudio, Popi, Tanaka, Testa, Jean, Jacqui, André, Camilo, Denise, Carol, Eduardão, César, ...

Ao Prof. Ricieri, que me mostrou a beleza da Matemática, e fez com que eu me apaixonasse por ela.

À Érika, a outra matemática por quem me apaixonei, e também à sua família, Sônia, Jair e Karina, que amorosamente me acolheram em seu lar.

E finalmente ao "mestre" Dantas, por me mostrar o Caminho. 


\begin{abstract}
This dissertation investigates biologically inspired models of pattern storage and retrieval, by means of feedback neural networks. This class of neural networks try to model some of the dynamical aspects involved in brain functioning. The study concentrated in Cellular Neural Networks, a local coupled version of the classical Hopfield model. The present research comprised stability analysis of the referred networks, as well as performance tests of various methods for content-addressable (associative) memory design in Cellular Neural Networks.
\end{abstract}




\section{Resumo}

Nessa dissertação, é investigado o armazenamento e a recuperação de padrões de forma biologicamente inspirada no cérebro. Os modelos estudados consistiram de redes neurais realimentadas, que tentam modelar certos aspectos dinâmicos do funcionamento do cérebro. Atenção especial foi dada às Redes Neurais Celulares, que constituem uma versão localmente acoplada do clássico modelo de Hopfield. Além da análise de estabilidade das redes consideradas, foi realizado um teste com o intuito de avaliar o desempenho de diversos métodos de construção de memórias endereçáveis por conteúdo (memórias associativas) em Redes Neurais Celulares. 



\section{Sumário}

Lista de Figuras $\ldots \ldots \ldots \ldots \ldots$. . . . . . . . . . . . . . . . . . . . . . . . . .

Lista de Tabelas . . . . . . . . . . . . . . . . . . . . . ix

1 Introdução 1

2 Sistemas dinâmicos 5

2.1 A teoria de sistemas dinâmicos . . . . . . . . . . . . . . . . . 5

2.2 Definições . . . . . . . . . . . . . . . . . . . . 6

2.3 Noções de dinâmica em sistemas discretos unidimensionais . . . . . . . . . . . 8

2.3.1 Dinâmica estacionária (ou de ponto fixo) . . . . . . . . . . . . 9

2.3.2 Dinâmica periódica . . . . . . . . . . . . . . . . . . . 9

2.3.3 Dinâmica quase-periódica . . . . . . . . . . . . . . . . 11

2.3.4 Dinâmica caótica . . . . . . . . . . . . . . . . . . 12

2.3 .5 Atratores . . . . . . . . . . . . . . . . . . 15

2.4 Estabilidade . . . . . . . . . . . . . . . . . . . 16

2.4.1 Estabilidade de pontos fixos . . . . . . . . . . . . . 16

2.4.2 Estabilidade de ciclos periódicos . . . . . . . . . . . . . . 18

2.4.3 Estabilidade estrutural e bifurcações . . . . . . . . . . . . . . . . 18

2.5 Sistemas contínuos n-dimensionais . . . . . . . . . . . . . . . 20

2.5.1 Sistemas lineares autônomos no plano . . . . . . . . . . . . . . 22

2.5.2 Estabilidade de sistemas lineares . . . . . . . . . . . . . . . 26

2.5.3 Linearização ou Método Indireto de Lyapunov . . . . . . . . . . . . 27

2.5.4 Segundo Método ou Método Direto de Lyapunov . . . . . . . . . . . . 29

2.6 Conclusão . . . . . . . . . . . . . . . . . . . . . . . 32

3 Redes Neurais Artificiais $\quad 33$

3.1 Princípio de funcionamento do neurônio . . . . . . . . . . . . . . . . . . . . 34

3.2 Modelo de neurônio de McCulloch e Pitts . . . . . . . . . . . . . . . . 34

3.3 O paradigma de Computação Neural . . . . . . . . . . . . . . . . 35

3.4 Modelos de Redes Neurais . . . . . . . . . . . . . . . . . . . . . . . 36 
3.5 Histórico de pesquisas na área . . . . . . . . . . . . . 38

3.6 Conclusão . . . . . . . . . . . . . . . . . . . . . . . . 38

4 O modelo de Hopfield 41

4.1 Equações da dinâmica . . . . . . . . . . . . . . . . . . . . . . . . 42

4.2 Análise de estabilidade . . . . . . . . . . . . . . . . . . . . . . 43

4.2 .1 Modelo contínuo . . . . . . . . . . . . . . . . . . . 43

4.2.2 Modelo discreto . . . . . . . . . . . . . . . . . . 45

4.3 Armazenamento de padrões . . . . . . . . . . . . . . . . . . . 46

4.4 Recuperação de padrões . . . . . . . . . . . . . . . . . . . . . . . . . 48

4.5 Simulação . . . . . . . . . . . . . . . . . . . . . . . . . . . 49

4.6 Discussão . . . . . . . . . . . . . . . . . . . . . 50

5 Redes Neurais Celulares $\quad 51$

5.1 Equações da dinâmica . . . . . . . . . . . . . . . . . . . . . . . 52

5.2 Análise de estabilidade . . . . . . . . . . . . . . . . . 53

5.3 Programação de uma RNC . . . . . . . . . . . . . . . . . . 54

5.4 RNCs para o processamento de imagens . . . . . . . . . . . . . . . 54

5.4.1 Máscaras ou moldes de clonagem . . . . . . . . . . . . . . . 55

5.4.2 Suavização (remoção de ruídos) em imagens . . . . . . . . . . . . 56

5.4 .3 Extração de contornos . . . . . . . . . . . . . . . . 58

5.5 Simulações . . . . . . . . . . . . . . . . . . . . . 60

5.5.1 Simulação 1: Remoção de ruído . . . . . . . . . . . . . . . . . 60

5.5.2 Simulação 2: Extração de contornos . . . . . . . . . . . . . . . 60

5.6 Discussão . . . . . . . . . . . . . . . . . . . . . . . 61

6 Memória associativa em RNCs 63

6.1 Formulação do problema . . . . . . . . . . . . . . . . . . . . 63

6.2 Método baseado em Matriz Pseudo-Inversa . . . . . . . . . . . . . . . . 65

6.2.1 Matriz de índice e restrição de conectividade de RNCs . . . . . . . . . 66

6.2.2 Estratégia de resolução revista . . . . . . . . . . . . . . . . . . 67

6.3 Método baseado em Decomposição por Valor Singular . . . . . . . . . . . 70

6.4 Método baseado em aprendizado Hebbiano . . . . . . . . . . . . . . 71

6.5 Método baseado em aprendizado por Perceptron . . . . . . . . . . . . . . . . . 74

6.5 .1 Perceptrons . . . . . . . . . . . . . . . . . . . . 74

6.5.2 Configuração de RNCs por Perceptrons . . . . . . . . . . . . . . 76

6.6 Método baseado em Inequações Matriciais Lineares . . . . . . . . . . . . . 76

6.6 .1 LMIs e GEVPs . . . . . . . . . . . . . . . . . . . . . . 78 
6.6.2 Estratégia de resolução utilizando LMIs . . . . . . . . . . . . . . . . 79

6.7 Discussão . . . . . . . . . . . . . . . . . . . . 81

7 Experimentos $\quad 83$

7.1 Características dos experimentos . . . . . . . . . . . . . . 83

7.2 Resultados . . . . . . . . . . . . . . . . . . . . . 86

7.3 Considerações Finais . . . . . . . . . . . . . . . . . . . 9 90

8 Conclusões $\quad 91$

Referências $\quad 98$

A Forma Canônica de Jordan 99

B Análise do modelo de RNCs 101

B.1 Introdução . . . . . . . . . . . . . . . . . . . . . . . . 101

B.2 Notação . . . . . . . . . . . . . . . . . . . . . . . . . . 101

B.3 Resultados . . . . . . . . . . . . . . . . . . . . . . . . 102

C Decomposição por Valor Singular 109

D Prova do Teorema 6.6.1 111

E $\quad$ Tabelas de resultados dos experimentos do Capítulo 7

E.1 Tabelas de resultados para ruído binário . . . . . . . . . . . . . . . . . . . . . 114

E.2 Tabelas de resultados para ruído gaussiano . . . . . . . . . . . . . . . . 117 



\section{Lista de Figuras}

2.1 Retroalimentação em um sistema dinâmico discreto. . . . . . . . . . . . . . . 7

2.2 Exemplo de dinâmica de ponto fixo de (2.6), para $A=2.8 \ldots \ldots$. . . . . . 9

2.3 Exemplo de dinâmica periódica de (2.6), para $A=3.4 \ldots \ldots$. . . . . . . 10

2.4 Dinâmica periódica de (2.9), para $\Omega=1 / 4$. (a) Trajetória do sistema; (b) Representação da trajetória em coordenadas polares, onde $\theta_{i}(i=1, \ldots, 4)$ denota os pontos que compõem o ciclo periódico de período 4, exibido pelo sistema. 11

2.5 Dinâmica quase-periódica de (2.9), para $\Omega=2 \pi$. (a) Trajetória do sitema; (b) Representação da trajetória em coordenadas polares, onde $S^{1}$ denota a circunferência de raio unitário. . . . . . . . . . . . . . . . . . . . 12

2.6 Exemplo de dinâmica caótica de (2.6), para $A=3.9$. A linha sólida corresponde à órbita com condição inicial $x(0)=0.10000$ e a linha pontilhada à

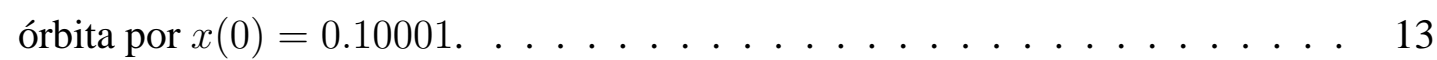

2.7 Expoente de Lyapunov de (2.6), para diferentes valores de $A$. . . . . . . . . 15

$2.8 N_{\epsilon}$ e $N_{\delta}$ denotam as vizinhanças de raio $\epsilon$ e $\delta$ em torno do ponto fixo $x^{*}$. . . . 17

2.9 Esboço numérico do diagrama de bifurcação de (2.6) . . . . . . . . . . . . . 20

2.10 Esboço do campo vetorial de (2.15), para $f(x)=\left(2 x_{1},-1 / 2 x_{2}\right) \ldots \ldots \ldots 21$

2.11 Caso 1 - autovalores reais e distintos. . . . . . . . . . . . . . . 25

2.12 Caso 2 - autovalores reais e iguais. . . . . . . . . . . . . 25

2.13 Caso 3 - autovalores complexos. . . . . . . . . . . . 26

3.1 Representação de um neurônio. . . . . . . . . . . . . . . . . . . . . . . . 34

3.2 Exemplos de funções de ativação. . . . . . . . . . . . . . . . . . 35

3.3 Esquema de rede tipo feedforward . . . . . . . . . . . . . . . 37

3.4 Esquema de rede tipo realimentada. . . . . . . . . . . . . . . . . . 37

4.1 Memórias armazenadas como pontos de equilíbrio. . . . . . . . . . . . . . 42

4.2 Gráfico de $\int_{0}^{v_{i}} h^{-1}(\eta) d \eta \ldots \ldots \ldots \ldots \ldots \ldots \ldots$

4.3 Gráfico de $h\left(u_{i}\right) \ldots \ldots \ldots \ldots \ldots \ldots \ldots$

4.4 Representação da função de energia $E(v) \ldots \ldots$. . . . . . . . . . . . . 46

4.5 Exemplo de padrões armazenados. . . . . . . . . . . . . . . . . . . . . 49 
4.6 Exemplo de recuperação de padrão com ruído. . . . . . . . . . . . . . . . . . 49

4.7 Exemplo de convergência para estado espúrio. . . . . . . . . . . . . . . . . 49

5.1 Representação espacial de uma RNC . . . . . . . . . . . . . . . . . . . 52

5.2 Exemplos de vizinhanças de uma célula. . . . . . . . . . . . . . . . . . . 53

5.3 (a) imagem original de entrada, em $t=0$; (b) imagem corrompida com $10 \%$ de ruído gaussiano ( $\sigma=1)$; (c) suavização pelo operador Laplaciano; (d) suavização por filtro passa-baixa. . . . . . . . . . . . . . . . . 61

5.4 (a) Extração de contornos pelo cálculo do gradiente; (b) Extração de contornos pelo operador Laplaciano; (c) Contornos obtidos com o cálculo do gradiente, após modificação; (d) Contornos obtidos com o operador Laplaciano, após modificação. . . . . . . . . . . . . . . . . . . . 62

6.1 Projeção de $X_{i}^{T}$ no subespaço $C o l$. . . . . . . . . . . . . . 66

7.1 Padrões utilizados para o treinamento da rede. . . . . . . . . . . . . . . 84

7.2 Exemplo de recuperação de padrão corrompido por ruído binário. . . . . . . . 84

7.3 Exemplo de recuperação de padrão corrompido por ruído gaussiano. . . . . . . . 84

7.4 Gráficos de desempenho para ruído binário. . . . . . . . . . . . . . 88

7.5 Gráficos de desempenho para ruído gaussiano. . . . . . . . . . . . . . . . . . . 89 


\section{Lista de Tabelas}

7.1 Melhores métodos - recuperação de padrões corrompidos por ruído binário. . . 90

7.2 Melhores métodos - recuperação de padrões corrompidos por ruído gaussiano. . 90

E.1 Desempenho dos métodos para padrões com ruído binário e vizinhança $\mathrm{r}=1$. . . 114

E.2 Desempenho dos métodos para padrões com ruído binário e vizinhança $r=2$. . 115

E.3 Desempenho dos métodos para padrões com ruído binário e vizinhança $\mathrm{r}=3$. . . 116

E.4 Desempenho dos métodos para padrões com ruído gaussiano e vizinhança $\mathrm{r}=1.117$

E.5 Desempenho dos métodos para padrões com ruído gaussiano e vizinhança $r=2$. 118

E.6 Desempenho dos métodos para padrões com ruído gaussiano e vizinhança r=3. 119 



\section{Capítulo 1}

\section{Introdução}

Uma das tarefas mais comuns a qual um computador digital é constantemente submetido, é o armazenamento e a recuperação de informações. Para realizar o endereçamento às informações armazenadas, um computador digital utiliza a localização física das mesmas, dentro da memória. Isto requer, por exemplo, que posições de memória sejam sistematicamente percorridas, caso se deseje procurar por um item armazenado. Conforme são exigidas maiores capacidades de armazenamento e recuperação de informações, o acesso aos itens armazenados torna-se extremamente ineficiente.

Observando a Natureza, por sua vez, nota-se que o problema de armazenamento e recuperação de informações é eficientemente resolvido no caso de seres vivos. Uma vez que estes devem ser capazes de lidar com padrões e situações complexas, provenientes do ambiente em que vivem, a Natureza proporcionou meios de lidar com toda essa complexidade. $\mathrm{O}$ animais classificados como "superiores" são dotados de um sistema nervoso bem elaborado, no qual essas tarefas referentes à memória são desempenhadas principalmente com a ajuda do cérebro.

O cérebro é considerado o principal órgão responsável pelo processamento de informação nos animais dotados de tal centro nervoso. Este órgão pode ser visto como um dispositivo capaz de processar sinais e, em geral, o faz de maneira muito superior a qualquer computador digital. Explora um paralelismo massivo por meio de bilhões de elementos processadores, os neurônios. Essas células nervosas, se individualmente consideradas, são dispositivos lentos e pouco confiáveis, comparados a circuitos de um computador digital. No entanto, funcionando de forma interdependente no cérebro, fazem deste um verdadeiro exemplo de Sistema Complexo $^{1}$.

A memória é talvez uma das mais notáveis tarefas que o cérebro é capaz de realizar, tanto em relação à capacidade de armazenamento, quanto em relação à capacidade de recuperação de informações. Ao contrário dos computadores digitais, que utilizam endereços físicos para

\footnotetext{
${ }^{1}$ Um sistema que exibe padrões complexos de comportamento, como caos, bifurcações e auto-organização.
} 
armazenar e recuperar informações, o cérebro é capaz de "lembrar" imagens, fatos, e outras informações armazenadas, apenas com base no conteúdo das mesmas. Por meio de pistas, que muitas vezes se constituem de itens de memória parciais ou inconsistentes, o cérebro é capaz de recuperar as informações originalmente armazenadas, de forma rápida e eficiente.

Com o objetivo de investigar modelos de memória inspirados no funcionamento do cérebro, e que possuam características de processamento similares, propõe-se, nessa dissertação, o estudo de Redes Neurais Artificiais (RNAs) que implementem modelos de memória endereçáveis por conteúdo (memórias associativas). As RNAs representam uma tentativa de se modelar, matematicamente, o processamento de informação da forma que, supostamente, este ocorre no cérebro. Assim, as redes neurais constituem um modelo de computação biologicamente inspirado no cérebro.

Diversas modelagens para redes neurais são possíveis. Visando especificamente o estudo de aspectos dinâmicos do funcionamento do cérebro, são consideradas, nesse estudo, redes neurais ditas realimentadas, compreendendo os modelos de Hopfield e de Redes Neurais Celulares (RNCs). Essas redes apreendem algumas das características observadas nos circuitos neurais reais, como a presença de retroalimentações entre os neurônios. Sendo tais redes modeladas como sistemas dinâmicos não-lineares, estas podem exibir certos comportamentos complexos típicos do cérebro, como oscilações, bifurcações e também o caos [Skarda \& Freeman, 1982].

Basicamente, os objetivos desejados com essa pesquisa compreendem:

(a) Investigação de modelos matemáticos e/ou computacionais de memórias associativas, inspiradas no funcionamento do cérebro;

(b) Análise de estabilidade de redes neurais realimentadas, modeladas como sistemas dinâmicos não-lineares;

(c) Estudo e comparação de desempenho entre diversos métodos de construção de memória associativa em RNCs.

Este trabalho se concentra em grande parte no modelo de RNCs, uma vez que esse modelo representa uma extensão do "clássico" modelo de Hopfield. Além disso, o estudo se baseia em conceitos fundamentais de sistemas dinâmicos, bem como em resultados mais avançados referentes à análise de sistemas não-lineares, que permitem que a estabilidade das redes consideradas seja analisada. Métodos de construção de memória associativa, envolvendo o modelo de RNCs, também são sistematicamente investigados nessa dissertação.

Essa pesquisa pode ser dividida em duas partes principais: uma teórica, compreendendo a análise de estabilidade de redes neurais realimentadas, e outra prática, envolvendo o teste de desempenho de memórias associativas em RNCs. As contribuições desse estudo concentram-se 
principalmente na parte prática, uma vez que não havia na literatura um estudo experimental criterioso e abrangente, envolvendo a avaliação de desempenho de diversos métodos de construção de memórias associativas em RNCs. Não menos importante, a parte teórica da pesquisa proporcionou organizar uma revisão detalhada de resultados de análise de modelos de redes neurais realimentadas.

O texto dessa dissertação foi organizado como segue. O Capítulo 2 aborda conceitos fundamentais de sistemas dinâmicos, além de resultados relevantes compreendendo a análise de sistemas não-lineares. O Capítulo 3 apresenta os princípios de funcionamento dos neurônios biológico e artificial, bem como noções básicas de Redes Neurais Artificiais. O Capítulo 4 revisa o modelo de Hopfield, e apresenta a análise de estabilidade do mesmo. O Capítulo 5 descreve o principal modelo de rede neural estudado nessa dissertação, as Redes Neurais Celulares, e aplicações destas para o processamento de imagens. O Capítulo 6 apresenta métodos de construção de memória associativa em RNCs. O Capítulo 7 compara, por meio de experimentos, o desempenho dos métodos apresentados no Capítulo 6. E, finalmente, o Capítulo 8 discute os principais resultados obtidos com esse estudo, sugerindo possíveis direções futuras para a pesquisa. 


\section{Capítulo 2}

\section{Sistemas dinâmicos}

Com a invenção do Cálculo Diferencial, por Newton e Leibnitz, a humanidade foi provida de um método matemático para descrever e analisar variações ou mudanças na Natureza. As equações diferenciais, que descrevem o tipo mais conhecido de sistema dinâmico, há muito têm sido utilizadas para expressar as leis de movimento em partículas e corpos celestes, entre eles o Sol e os planetas. Com o passar do tempo, cientistas começaram a estender seus modelos para descrever outros tipos de sistemas e fenômenos mais complexos. Assim, as equações diferenciais se tornaram uma linguagem comum da ciência para descrever e estudar os mais variados fenômenos que evoluem no tempo, sejam eles físicos, econômicos, sociais, biológicos, entre outros.

\subsection{A teoria de sistemas dinâmicos}

As origens da teoria dos sistemas dinâmicos são atribuídas ao estudo de sistemas físicos, provenientes principalmente de problemas em mecânica celeste, como o problema dos três corpos. Esse problema foi uma extensão do problema dos dois corpos, resolvido por Newton, que consistia em se calcular o movimento da Terra ao redor do Sol, dada a força de atração gravitacional entre ambos [Strogatz, 1989]. O problema dos três corpos procura determinar o comportamento das órbitas de três corpos celestes, como exemplo, o Sol, com dois planetas orbitando ao seu redor. Foi o trabalho de Henry Poincaré, no século XIX, que detectou pela primeira vez a existência de soluções complexas, que atualmente diriam-se caóticas, para as equações de movimento obtidas.

A teoria de sistemas dinâmicos visa a compreensão, de forma qualitativa ou geométrica, de soluções para sistemas de equações diferenciais ou de diferenças. Não se procura obter soluções explícitas para as equações, mas sim estudar sistemas mais complexos, cujas soluções são difíceis de se interpretar ou, ainda, sistemas para os quais soluções não são possíveis de 
serem obtidas usando-se as técnicas tradicionais de solução. O objetivo principal da teoria é deduzir propriedades das soluções, tais como: determinar a estabilidade do sistema, perante perturbações; prever seu comportamento a longo prazo; analisar a ocorrência de mudanças (bifurcações) de comportamento, entre outras.

Devido à teoria de sistemas dinâmicos se tratar de uma sub-área muito abrangente e genérica da Matemática, a exposição apresentada nesse capítulo se concentrará apenas no estudo de sistemas dinâmicos descritos ou operando em $\mathbb{R}^{n}$. Este tipo de sistema é suficiente para descrever a maioria dos sistemas físicos de interesse. Normalmente, faz-se uma distinção entre sistemas dinâmicos cuja variável independente (que geralmente representa o tempo) assume valores contínuos e discretos. No primeiro caso, as equações envolvidas serão equações diferenciais ordinárias e, no segundo, equações de diferenças. A partir de agora no texto, o termo sistema é usado para designar um sistema dinâmico definido em $\mathbb{R}^{n}$ ou em um subconjunto deste espaço. O termo sistema contínuo, por sua vez, denotará um sistema cuja variável independente (tempo) assume valores contínuos, e sistema discreto, por sua vez, denotará um sistema cuja variável independente assume valores discretos.

Na Seção 2.2, algumas definições elementares são introduzidas. Na Seção 2.3, conceitos preliminares de dinâmica em sistemas discretos são revistos, como o conceito de trajetória, condição inicial, entre outros. Os diferentes tipos de dinâmica existentes também são abordados, no caso de sistemas discretos. Considerações sobre estabilidade são feitas na Seção 2.4, assim como uma breve introdução ao estudo de bifurcações. Em seguida, na Seção 2.5, resultados já vistos para sistemas discretos são estendidos para o caso de sistemas contínuos, sendo que outros métodos de análise de estabilidade são também apresentados.

\subsection{Definições}

Um sistema dinâmico é uma descrição matemática de como um sistema evolui no tempo, por meio de equações diferenciais, ou equações de diferenças [Ott, 1993]. Em termos físicos, um exemplo de sistema dinâmico é aquele formado por uma partícula de massa pontual, que se desloca no espaço. A descrição matemática da lei de evolução dessa partícula pode ser feita, por exemplo, por meio de equações diferenciais, que descrevem como ela se movimenta no espaço, ou seja, que descrevem como o estado do sistema evolui no tempo. Por estado do sistema, entende-se o conjunto de informações necessárias, em um dado instante de tempo, para caracterizar o comportamento do mesmo. No exemplo considerado, o estado pode envolver informações como a exata localização da partícula no espaço (em termos de coordenadas físicas cartesianas), como também taxas de variações (ou derivadas) dessas quantidades como, por exemplo, a velocidade da partícula.

O ingrediente principal de um sistema dinâmico é a retroalimentação (feedback), como ilus- 


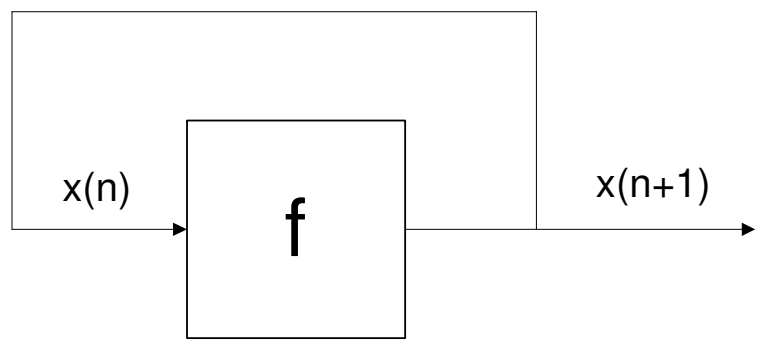

Figura 2.1: Retroalimentação em um sistema dinâmico discreto.

tra a Figura 2.1. O estado do sistema, em um dado instante de tempo, é dado em função do estado (ou estados) passados do mesmo. Dessa forma, diz-se que o sistema possui memória. Sua descrição pode ser feita por meio da equação:

$$
x(n+1)=f(x(n)),
$$

onde $n \in B(B \subset \mathbb{Z})$ denota o tempo, $x: B \rightarrow \mathbb{R}$ é o estado do sistema e $f: \mathbb{R} \rightarrow \mathbb{R}$ é a lei de evolução do mesmo. A equação de diferenças (2.1) descreve um sistema discreto ou mapa. Por outro lado, se a variável independente admite valores contínuos, tem-se um sistema contínuo ou fluxo, dado pela equação diferencial:

$$
\dot{x}=f(x(t))
$$

onde $t \in I(I \subset \mathbb{R}), x: I \rightarrow \mathbb{R}, f: \mathbb{R} \rightarrow \mathbb{R}$ é diferenciável e $\dot{x}$ denota a derivada $\frac{d x}{d t}$.

Estas formulações podem ser estendidas ou generalizadas para casos em que o estado do sistema envolva $N>1$ variáveis ou graus de liberdade, isto é:

$$
\left\{\begin{array}{c}
x_{1}(n+1)=f_{1}\left(t, x_{1}(n), x_{2}(n), \ldots, x_{N}(n)\right), \\
x_{2}(n+1)=f_{2}\left(t, x_{1}(n), x_{2}(n), \ldots, x_{N}(n)\right), \\
\vdots \\
x_{N}(n+1)=f_{N}\left(t, x_{1}(n), x_{2}(n), \ldots, x_{N}(n)\right) .
\end{array}\right.
$$

ou, em notação vetorial:

$$
x(n+1)=f(t, x(n)),
$$

onde $x=\left[x_{1}, \ldots, x_{N}\right]^{T}$ e $f=\left[f_{1}\left(t, x_{1}, \ldots, x_{N}\right), \ldots, f_{N}\left(t, x_{1}, \ldots, x_{N}\right)\right]^{T}$.

As mesmas definições são válidas para equações diferenciais, que em notação vetorial fica:

$$
\frac{d x}{d t}=f(t, x(t))
$$


Nas definições acima, o vetor $x \in \mathbb{R}^{N}$ denota o estado do sistema, que está contido no chamado espaço de estados ${ }^{1}$ do mesmo. Por exemplo, no caso do sistema que descreve o deslocamento de uma partícula no espaço, como anteriormente mencionado, poder-se-ia descrevê-lo com três graus de liberdade, isto é, seu espaço de estados seria definido por $x=\left[x_{1}, x_{2}, x_{3}\right]^{T}$, que representa as coordenadas cartesianas da partícula no espaço.

\subsection{Noções de dinâmica em sistemas discretos unidimensio- nais}

Quando se estuda um sistema dinâmico, geralmente se está interessado em compreender o comportamento final (ou assintótico) do mesmo [Devaney, 1989]. Isto é, deseja-se conhecer como o sistema se comporta, conforme o tempo evolui. Considere, para efeito de exemplo, o sistema descrito pela equação (2.1). Uma forma de enxergar seu comportamento, é escolhendo um valor inicial $x(0)$ para $x(t)$, e calculando seus estados seguintes no tempo, assim:

$$
x(0), f(x(0)), f(f(x(0))), f(f(f(x(0)))), \ldots
$$

À sequiência de valores acima dá-se o nome de órbita do sistema dinâmico (2.1) por $x(0)$. $\mathrm{O}$ valor de $x$ para $n=0$ (isto é, $x(0)$ ) é chamado condição inicial do sistema. A designação órbita se deve a uma possível interpretação física dessa sequiência numérica gerada: é como se ela descrevesse o movimento de uma partícula de massa pontual, se movimentando no espaço.

A órbita, obtida a partir da iteração de (2.1), depende da escolha da lei $f$, o que pode dar origem a diferentes tipos de comportamento. Se $f$ for uma função linear em $x$, esta dinâmica é bem simples e $x \rightarrow 0$ ou $\infty$, dependendo de $f$. Neste caso, existem muitas ferramentas para analisar o comportamento resultante do sistema. Mas observa-se que, na Natureza, a maioria dos fenômenos ou leis são não-lineares, o que torna a análise dos mesmos complexas, requerendo o uso de ferramentas mais elaboradas.

Para ilustrar a riqueza de comportamentos gerados por um sistema não-linear, considere o mapa quadrático ou logístico [Strogatz, 1989]:

$$
x(n+1)=A x(n)(1-x(n)),
$$

onde $n \in D,(D \subset \mathbb{Z}), x: D \rightarrow \mathbb{R}$ e $A \in \mathbb{R}$ é um parâmetro. Dependendo do valor que esse parâmetro $A$ assume em (2.6), certos tipos de dinâmica podem ser observados, como investigado a seguir.

\footnotetext{
${ }^{1}$ Quando em duas dimensões $(N=2)$, diz-se plano de fase do sistema.
} 


\subsubsection{Dinâmica estacionária (ou de ponto fixo)}

Considere a equação (2.6), para $A=2.8$ :

$$
x(n+1)=2.8 x(n)(1-x(n))
$$

Seja uma órbita desse sistema, sob a condição inicial $x(0)=0.1$, por exemplo. A trajetória (ou série temporal) na Figura 2.2 ilustra o comportamento dessa órbita.

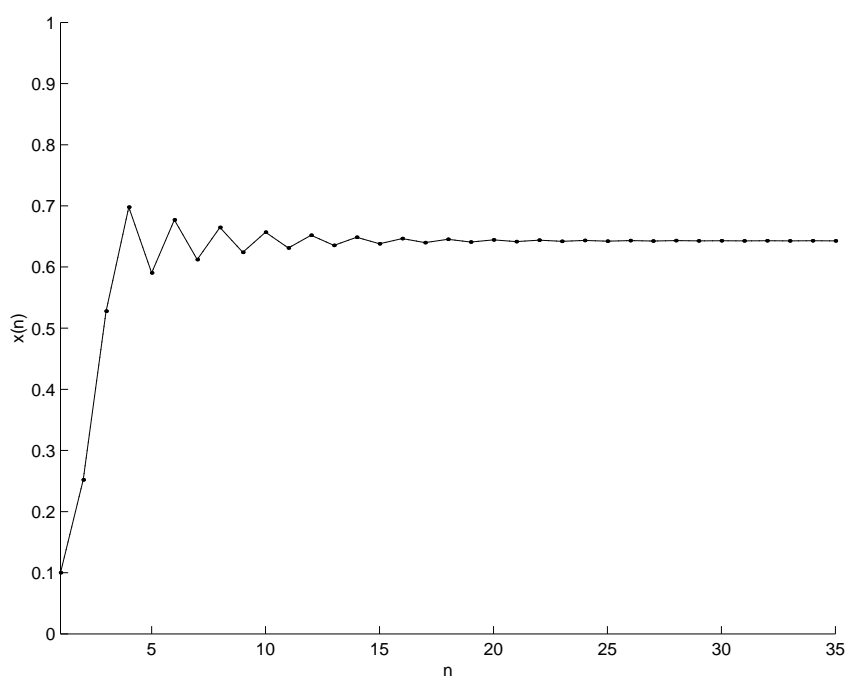

Figura 2.2: Exemplo de dinâmica de ponto fixo de (2.6), para $A=2.8$.

Nesse caso, observa-se que a iteração leva o estado do sistema tender a um valor que se repete indefinidamente, conforme o tempo prossegue (isto é, $x(n) \rightarrow x^{*}=0.643$, quando $n \rightarrow \infty)$. A esse estado $x^{*}$ que atrai a órbita do sistema, dá-se o nome de ponto fixo.

Definição 2.3.1 Um ponto $x^{*} \in \mathbb{R}$ de (2.1) é dito um ponto fixo de $f$, se

$$
f\left(x^{*}\right)=x^{*} .
$$

Ou seja, um ponto fixo é uma solução constante de (2.1):

$$
x(n+1)=x(n) .
$$

\subsubsection{Dinâmica periódica}

Considere, agora, a equação (2.6), com o parâmetro $A=3.4$ : 


$$
x(n+1)=3.4 x(n)(1-x(n)) .
$$

A órbita por $x(0)=0.1$, por exemplo, dá origem à trajetória ilustrada na Figura 2.3. Em casos como esse, onde as órbitas do sistema dinâmico tendem a repetição sucessiva de uma seqüência de valores, dá-se o nome de ciclo periódico ou ciclo limite.

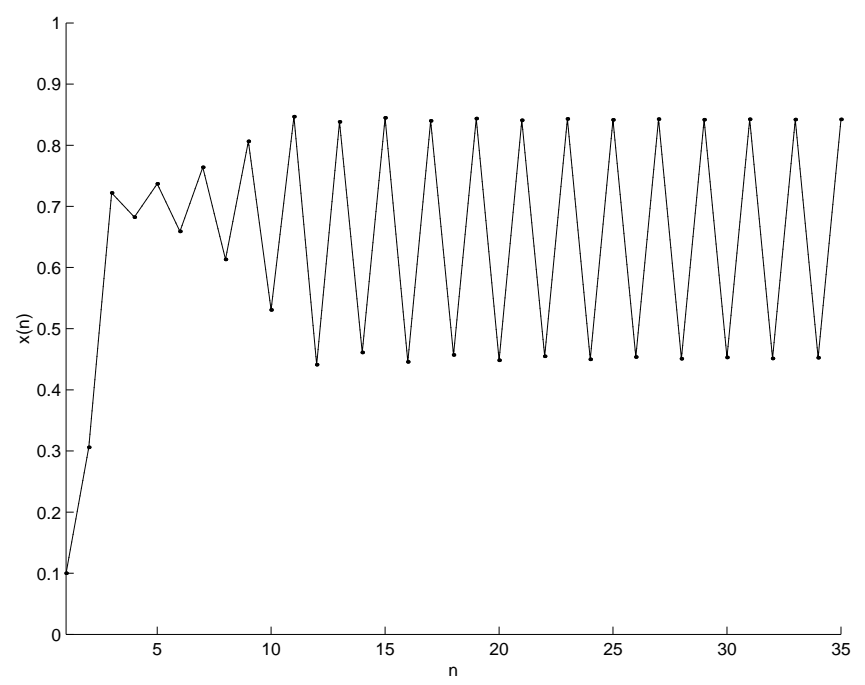

Figura 2.3: Exemplo de dinâmica periódica de (2.6), para $A=3.4$.

Uma solução periódica ou ciclo limite de (2.1) é tal que:

$$
x(n+P)=x(n),
$$

para algum inteiro positivo $P$, denominado o período da oscilação que se repete. Cada ponto deste ciclo é dito um ponto periódico, como afirma a definição seguinte.

Definição 2.3.2 Um ponto b de (2.1) é dito periódico se

$$
f^{P}(b)=b, \text { para algum inteiro } P>0 .
$$

onde $f^{P}$ denota a composição

$$
f^{P}=f \circ f \circ \cdots \circ f(P \text { vezes })
$$

Pela Definição 2.3.2, percebe-se que um ponto fixo é um caso particular de dinâmica periódica, para o período $P=1$ (comparar com a Definição 2.3.1). 


\subsubsection{Dinâmica quase-periódica}

Considere agora outro mapa, o chamado mapa do círculo:

$$
\theta(n+1)=\theta(n)+\Omega \bmod 1 .
$$

que descreve a variação do ângulo $\theta$ ao longo de um círculo de raio unitário. Note que a variável $\theta(n)$ é restrita ao intervalo $[0,1]$, pela operação $\bmod 1$. O parâmetro $\Omega$ representa uma freqüência externa que é aplicada.

Se o parâmetro $\Omega$ for um número racional $\Omega=p / q$, onde $p$ e $q$ são inteiros, tem-se que o mapa dado por (2.9) possui um ciclo periódico, de período $q$ (ver Figura 2.4(a)). Mas se, ao contrário, $\Omega$ for um número irracional, não podendo ser decomposto na forma $p / q$, então os valores de $\theta(n)$ nunca se repetirão, para $n \rightarrow \infty$ (ver Figura 2.5(a)). Esse tipo de comportamento recebe o nome de quase-periódico (pois se assemelha a periódico). Quando o sistema dado por (2.9) exibe quase-periodicidade, a representação de uma trajetória deste, em coordenadas polares, dá origem a uma sequiência de valores de $\theta(n)$ que preenche de forma densa a circunferência de raio unitário (Figura 2.5(b)). Uma trajetória periódica de (2.9), por sua vez, apenas visita um número finito de pontos da circunferência (Figura 2.4(b)).

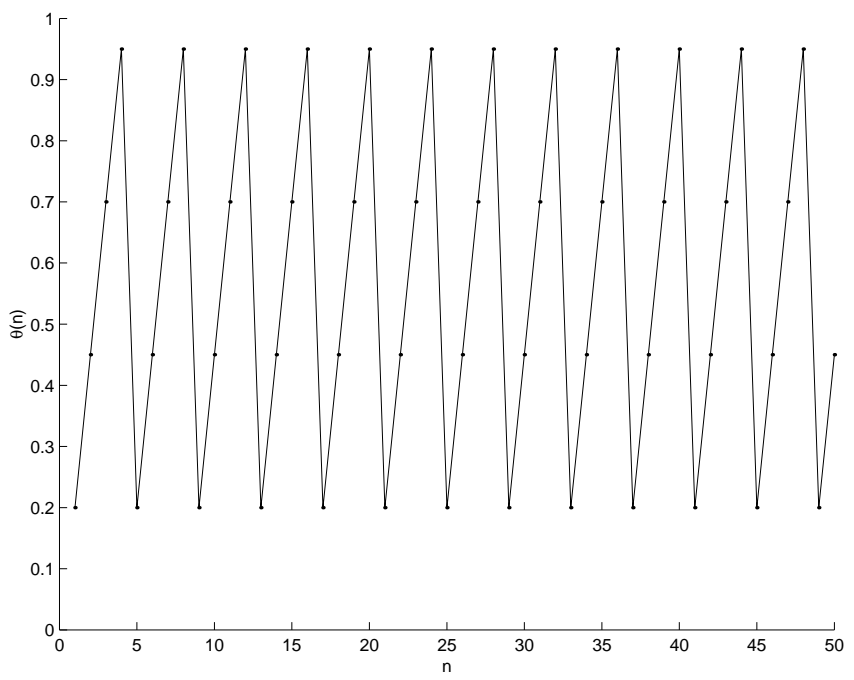

(a)

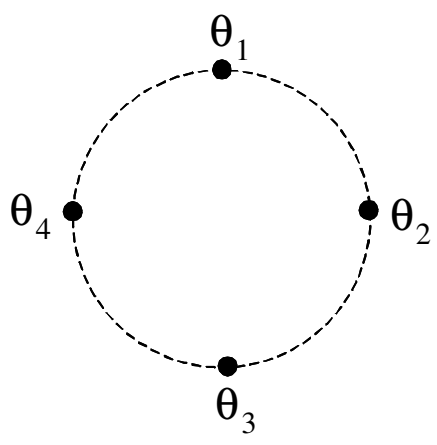

(b)

Figura 2.4: Dinâmica periódica de (2.9), para $\Omega=1 / 4$. (a) Trajetória do sistema; (b) Representação da trajetória em coordenadas polares, onde $\theta_{i}(i=1, \ldots, 4)$ denota os pontos que compõem o ciclo periódico de período 4 , exibido pelo sistema. 


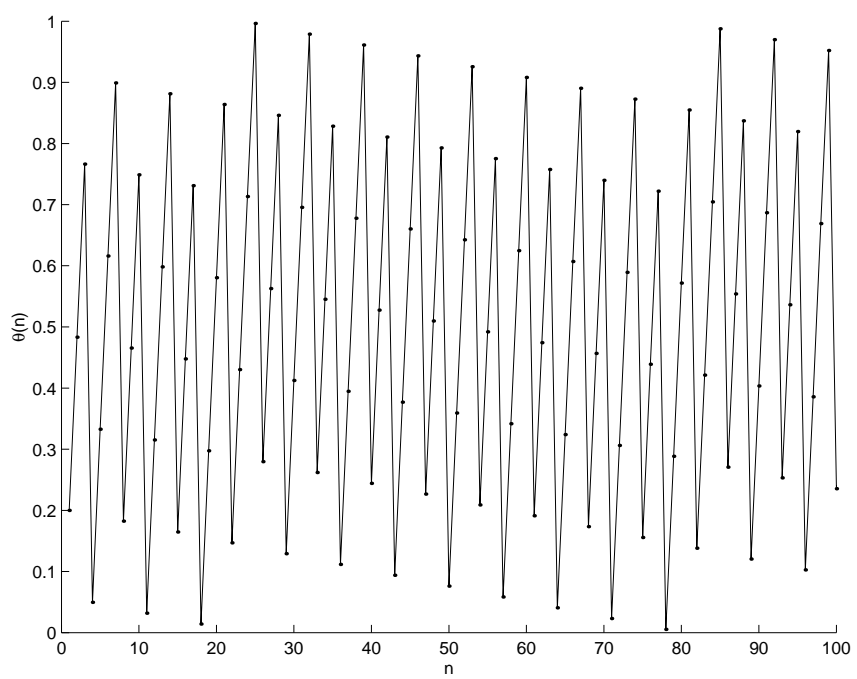

(a)

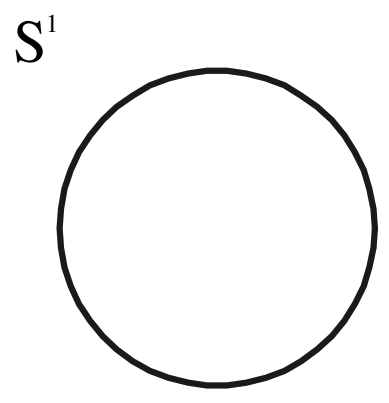

(b)

Figura 2.5: Dinâmica quase-periódica de (2.9), para $\Omega=2 \pi$. (a) Trajetória do sitema; (b) Representação da trajetória em coordenadas polares, onde $S^{1}$ denota a circunferência de raio unitário.

\subsubsection{Dinâmica caótica}

Considere, novamente, o mapa logístico (2.6), mas agora com o parâmetro $A=3.9$ :

$$
x(n+1)=3.9 x(n)(1-x(n)) .
$$

Na Figura 2.6, pode-se ver as trajetórias resultantes de duas órbitas com condições iniciais $x(0)=0.10000$ e $x(0)=0.10001$., respectivamente, sob ação da lei (2.10).

As órbitas ilustram um tipo de comportamento irregular, que se assemelha a aleatório. No entanto, elas foram geradas por uma lei determinística, dada pela equação (2.10). Outra característica das órbitas é que elas são limitadas, isto é, não divergem para infinito. Além disso, embora iniciando-se muito próximas uma da outra, as órbitas se afastam continuamente após um certo intervalo de tempo. O tipo de comportamento observado no sistema é o que se chama de caos na literatura.

\section{O descobrimento do caos}

A existência da dinâmica caótica em equações diferenciais, foi pela primeira vez relatada por Henry Poincaré, no século XIX. Entretanto, como não existiam computadores naquela época, Poincaré não pôde demonstrar sua "descoberta", pois era impraticável se efetuar os cálculos de uma órbita do sistema. $\mathrm{O}$ assunto foi então esquecido, por quase um século.

Com o advento do computador digital, a possibilidade de simulação de sistemas dinâmicos tornou-se viável, e a pesquisa nessa área teve um sensível desenvolvimento. Edward Lorenz, 


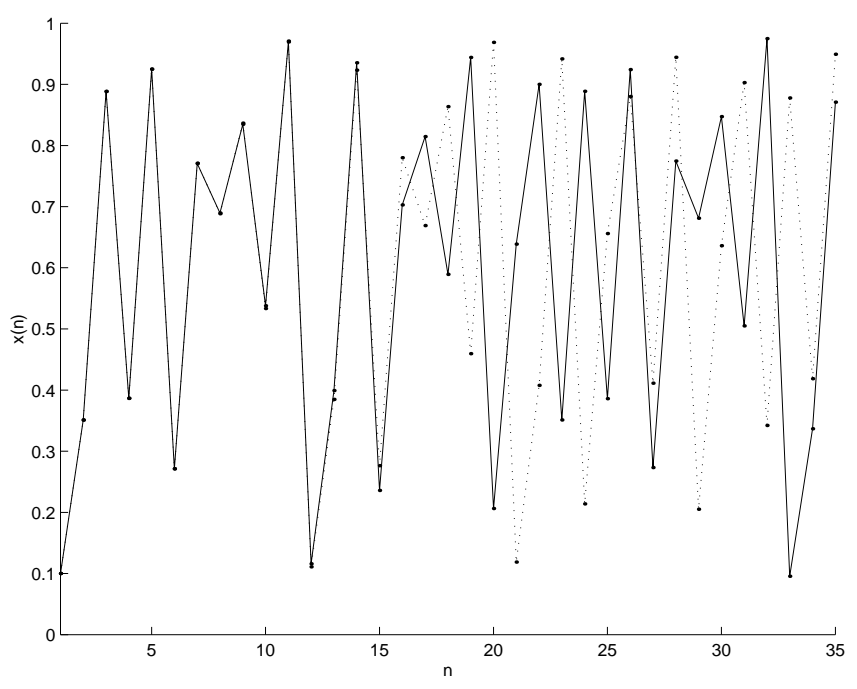

Figura 2.6: Exemplo de dinâmica caótica de (2.6), para $A=3.9$. A linha sólida corresponde à órbita com condição inicial $x(0)=0.10000$ e a linha pontilhada à órbita por $x(0)=0.10001$.

um pesquisador que estudava modelos metereológicos, foi o responsável pelo resurgimento do interesse pelo estudo do caos. Ele tentava simular um de seus modelos no computador, quando se deparou com resultados "estranhos". Escolhendo uma certa condição inicial para efetuar um experimento com seu modelo, este produzia uma certa previsão climática como uma série temporal irregular e não periódica. Entretanto, tentando reexecutar seu experimento, escolheu uma condição inicial muito próxima à primeira, obtendo aparentemente o mesmo resultado, durante as primeiras iterações. Curiosamente, após mais algumas iterações, a órbita obtida divergia completamente daquela obtida no experimento inicial.

Anos mais tarde, se descobriu que seus resultados estavam corretos, assim como estava certo Poincaré. Na verdades, com a pesquisa descobriu-se que o comportamento caótico é muito comum, e pode existir em modelos muitos simples, como é o caso do mapa logístico estudado nas seções anteriores.

\section{Características do caos}

Uma das características observadas em sistemas caóticos é o que se chama de sensibilidade às condições iniciais, ou efeito borboleta. Esse efeito é resultado de duas órbitas muito próximas do sistema que divergem completamente, conforme o sistema evolui no tempo. É chamado de efeito borboleta por uma analogia em relação ao modelo de Lorenz, que indicou que uma pequena perturbação, como o simples bater de asas de uma borboleta, poderia alterar completamentamente a previsão metereológica a longo, devido à sensibilidade às condições iniciais observadas no modelo.

Apesar do comportamento aparentemente randômico, um sistema caótico é, como todo sis- 
tema dinâmico, resultado de uma lei determinística de evolução (isto é, sempre o mesmo comportamento é gerado, sob as mesmas condições). Essa aperiodicidade, que mais lembra um sistema estocástico, é o que impossibilita previsões a longo prazo a respeito do estado do sistema. É interessante observar que uma órbita caótica visita sempre um ponto distinto do espaço de estados, nunca passando pelo mesmo ponto mais do que uma vez, em qualquer instante de tempo. Além disso, as órbitas são limitadas, não ocorrendo divergência do estado para infinito, mesmo com esse comportamento complexo. O estudo e a previsão do comportamento caótico só pode então ser feito por meio de medidas estatísticas, cujo estudo dá origem à chamada Teoria Ergódica [Jr. \& de Melo, 1982]. Uma abordagem mais formal, acerca de sistemas caóticos, pode ser encontrada em [Devaney, 1989].

Uma das maneiras estatísticas de se investigar o comportamento caótico de um sistema, é através do cálculo dos expoentes de Lyapunov do mesmo. Estes valores medem o grau de divergência entre órbitas inicialmente próximas, sendo calculados por:

$$
\lambda_{N}=\lim _{k \rightarrow \infty} \frac{1}{k} \sum_{i=1}^{k} \ln \left|f^{\prime}(x(i))\right|
$$

onde $k$ indica o número de iterações em que o sistema é observado, $f^{\prime}$ é a derivada da lei de evolução do sistema, que é calculada em cada ponto $x(i), i=1, \ldots, k$ de uma órbita gerada sob uma condição inicial qualquer. Os expoentes de Lyapunov são calculados para cada uma das $N$ dimensões ou variáveis de estado do sistema considerado.

Valores positivos para os expoentes de Lyapunov (ao menos um deles sendo positivo já é suficiente) indicam o caos. Valores negativos indicam comportamentos convergentes, como periódico ou de ponto fixo. Valores nulos indicam quase-periodicidade ou mudanças qualitativas de comportamento (pontos de bifurcação), conforme discutidos mais adiante no texto (ver Seção 2.4.3).

Uma forma interessante de se analisar o comportamento de um sistema dinâmico, é calculando os expoentes de Lyapunov do mesmo, para diferentes valores dos parâmetros. Uma ilustração desse procedimento de análise é dada na Figura 2.7, onde os valores para o único expoente de Lyapunov de (2.6) são plotados no eixo- $y$, variando-se no eixo- $x$ os valores de $A$.

O diagrama da Figura 2.7 caracteriza os diferentes tipos de dinâmica para o mapa logístico (equação (2.6)). Para $A=2.8$ e $A=3.4$, por exemplo, o expoente é negativo, indicando convergência de órbitas. Nesse caso, a dinâmica do sistema é de ponto fixo e periódica, respectivamente, conforme se verificou nas Seções 2.3.1 e 2.3.2. Para $A=3.9$, por exemplo, o expoente é positivo, caracterizando a divergência das órbitas, o que verificou-se como a presença do caos, no início da seção. 


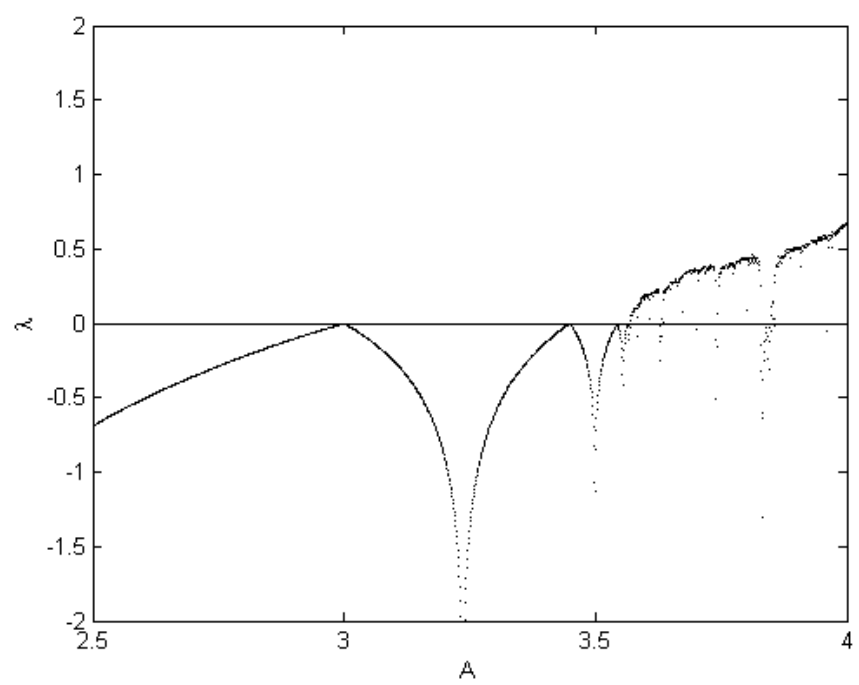

Figura 2.7: Expoente de Lyapunov de (2.6), para diferentes valores de $A$.

\subsubsection{Atratores}

As regiões do espaço de estados para as quais as órbitas do sistema convergem, são denominadas atratores. Por exemplo, o estado $x=0.643$ em (2.7) é um atrator do tipo ponto fixo. Sistemas com dinâmica periódica, como (2.8), apresentam atratores ditos periódicos ou ciclos limites. Mesmo os sistemas caóticos, que não apresentam convergência de órbitas, podem apresentar uma espécie de "atrator" denominado "atrator estranho", que são regiões limitadas do espaço de estados onde as órbitas caóticas permanecem contidas. O conjunto de todos os pontos no espaço de estados que convergem para um determinado atrator, recebe o nome de bacia de atração desse atrator.

Sistemas dinâmicos que possuem atratores são ditos sistemas dissipativos. Geometricamente, esses sistemas sofrem contrações em volumes do espaço de estados. Isto é, se for escolhida uma região desse espaço cujos pontos são usados como condições iniciais do sistema, percebe-se que conforme o estado evolui, as órbitas geradas convergem ou tendem para regiões menores (em termos de volume) do que a região que inicialmente as geraram. A denominação de sistemas dissipativos provém de uma analogia desses com sistemas físicos que sofrem dissipação de energia.

Outra categoria de sistemas são os ditos conservativos ou Hamiltonianos, que, diferentemente dos dissipativos, preservam volumes do espaço de estados. Este é o caso daqueles sistemas que exibem dinâmica quase-periódica, como apresentado na Seção 2.3.3. O que caracteriza este tipo de sistema é que não há convergência nem divergência das órbitas, como se o sistema sempre conservasse sua "energia". Um exemplo de sistema conservativo é um pêndulo que não sofre ação de atrito e, portanto, oscila indefinidamente (se a posição inicial não for a de 
repouso).

\subsection{Estabilidade}

Outro conceito importante no estudo de sistemas dinâmicos é o conceito de estabilidade. Um sistema é dito estável se, sob a ação de uma perturbação (limitada), este a amortece, não propagando demasiadamente seus efeitos sobre o comportamento do mesmo. Assim, um sistema é considerado estável se, quando ligeiramente perturbado, retorna ao seu estado original ou próximo a este, depois de um certo tempo transcorrido.

Mais estritamente, o conceito de estabilidade é uma propriedade de pontos do espaço de estados, ao invés de uma propriedade de um sistema em si. Assim, diz-se que um ponto fixo é estável ou instável, ou que um ciclo limite possui a propriedade de estabilidade ou instabilidade. A seguir, são apresentados teoremas resultantes da análise de estabilidade de ponto fixos e periódicos, para sistemas discretos. Essa exposição se restringirá, nesse momento, a sistemas unidimensionais, sendo postergadas as considerações para sistemas contínuos e de maior dimensão.

\subsubsection{Estabilidade de pontos fixos}

Um ponto fixo é dito estável se soluções iniciando-se próximo a esse (em uma certa vizinhança), permanecem próximas (nessa mesma vizinhança), conforme o tempo prossegue. Isto é o que afirma a próxima definição.

Definição 2.4.1 Um ponto fixo $x^{*}$ de (2.1) é estável se, para toda vizinhança $N_{\epsilon}$ de $x^{*}$, existe uma vizinhança $N_{\delta}$ de $x^{*}$ em $N_{\epsilon}$, tal que toda solução $x(n)$ com $x(0)$ em $N_{\delta}$ é definida $e$ permanece em $N_{\epsilon}$, para todo $n>0$ (ver Figura 2.8(a)).

Definição 2.4.2 Se além de estável, conforme a Definição 2.4.1, toda solução de (2.1) iniciando em $N_{\delta}$ tende a $x^{*}$, isto é, se $\lim _{n \rightarrow \infty} x(n)=x^{*}$, então $x^{*}$ é dito assintoticamente estável (ver Figura 2.8(b)).

Definição 2.4.3 Um ponto fixo $x^{*}$ de (2.1) é dito instável se ele não for estável. Isso significa que existe ao menos uma vizinhança $N_{\epsilon}$ de $x^{*}$ tal que, para toda vizinhança $N_{\delta}$ de $x^{*}$ em $N_{\epsilon}$, existe ao menos uma solução $x(n)$ que se inicia em $x(0) \in N_{\delta}$, mas que não permanece em $N_{\epsilon}$ (ver Figura 2.8(c)).

O teorema, apresentado a seguir, estabelece um critério para se determinar a estabilidade assintótica de um ponto fixo. 
Teorema 2.4.4 Seja $x^{*}$ um ponto fixo de (2.1). A seguinte proposição é então verdadeira:

(a) Se $\left|f^{\prime}\left(x^{*}\right)\right|<1$, então $x^{*}$ é assintoticamente estável.

(b) $\operatorname{Se}\left|f^{\prime}\left(x^{*}\right)\right|>1$, então $x^{*}$ é instável.

Prova: A prova desse teorema está além do escopo deste texto, podendo ser encontrada em [Elaydi, 1999].

Observação 2.4.5 O Teorema 2.4.4 é válido somente para pontos ditos hiperbólicos, isto é, em que $\left|f^{\prime}\left(x^{*}\right)\right| \neq 1$. Caso $\left|f^{\prime}\left(x^{*}\right)\right|=1$, uma análise mais detalhada de (2.1) faz-se necessária.

Para ilustrar como o Teorema 2.4.4 pode ser utilizado, considere o seguinte exemplo.

Exemplo 2.4.6 Determinar a estabilidade dos pontos fixos de (2.6), para $A=2$.

Solução: De (2.6), a equação do sistema é:

$$
x(n+1)=2 x(n)(1-x(n))
$$

1. Iguala-se $x(n+1)=x(n)$ e resolve-se a equação resultante:

$$
x=2 x(1-x) .
$$

Assim, $x_{1}=0$ e $x_{2}=\frac{1}{2}$ são soluções e, portanto, pontos fixos de (2.12).

2. Para aplicar os critérios de estabilidade do Teorema 2.4.4, faz-se necessário o cálculo da derivada de $f$

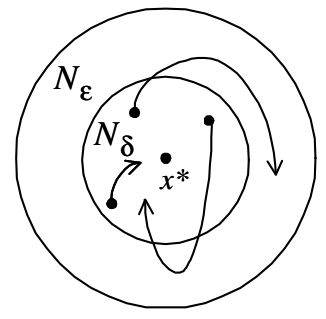

(a) Estável

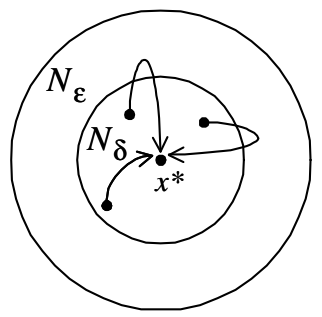

(b) Assintoticamente estável

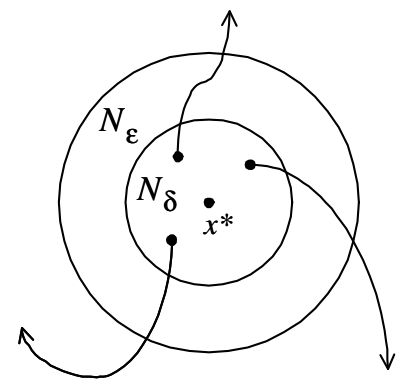

(c) Instável

Figura 2.8: $N_{\epsilon}$ e $N_{\delta}$ denotam as vizinhanças de raio $\epsilon$ e $\delta$ em torno do ponto fixo $x^{*}$. 


$$
f^{\prime}(x)=-4 x+2
$$

que aplicada nos pontos fixos encontrados, permite concluir:

(a) O ponto fixo $x_{1}=0$ é instável, pois $f^{\prime}(0)=2>1$;

(b) O ponto fixo $x_{2}=\frac{1}{2}$ é assintoticamente estável, pois $f^{\prime}\left(\frac{1}{2}\right)=0<1$.

\subsubsection{Estabilidade de ciclos periódicos}

De forma análoga a pontos fixos, as mesmas questões sobre estabilidade surgem para sistemas com dinâmica periódica. A estabilidade de um ciclo periódico é definida em função da estabilidade dos pontos que compõem a órbita, como afirma o teorema seguinte. Este teorema é, na verdade, uma extensão do Teorema 2.4.4, aplicando-se a regra da cadeia.

Teorema 2.4.7 Seja $\Omega=\{x(0), x(1), \ldots, x(P-1)\}$ um ciclo periódico de (2.1), com período P. Pode-se afirmar que:

(a) O ciclo periódico $\Omega$ é assintoticamente estável se

$$
\left|f^{\prime}(x(0)) \cdot f^{\prime}(x(1)) \cdot \ldots \cdot f^{\prime}(x(k-1))\right|<1
$$

(b) O ciclo $\Omega$ é instável se

$$
\left|f^{\prime}(x(0)) \cdot f^{\prime}(x(1)) \cdot \ldots \cdot f^{\prime}(x(k-1))\right|>1
$$

Prova: A prova desse teorema esta além do escopo deste texto, podendo ser encontrada em [Elaydi, 1999].

Observação 2.4.8 O Teorema 2.4.7 nada afirma sobre a estabilidade de $\Omega$ quando o produto das derivadas, calculadas nos pontos da órbita periódica, é igual a 1. Nesse caso, uma análise mais detalhada de f faz-se necessária.

\subsubsection{Estabilidade estrutural e bifurcações}

Outro tipo de estabilidade que pode ser discutida a respeito de um sistema dinâmico é a estabilidade estrutural. Um sistema é dito estruturalmente estável se não tem seu comportamento significativamente alterado, quando seus parâmetros são perturbados ou variados. Em um sistema desse tipo, não ocorrem bifurcações ou mudanças qualitativas de comportamento, 
como, por exemplo, uma mudança de dinâmica de ponto fixo para periódica. O objetivo da Teoria de Bifurcações é descrever os tipos de mudança de comportamento que sistemas dinâmicos sofrem, e como essas mudanças ocorrem, quando seus parâmetros são variados.

O cérebro, por exemplo, se visto como um sistema dinâmico não-linear, pode fazer uso desse mecanismo de bifurcação para alterar entre diferentes tipos de comportamentos desejáveis. Como exemplo, têm-se os estudos realizados por W. J. Freeman e colaboradores [Skarda \& Freeman, 1982] no condicionamento de coelhos com o uso de odores. Freeman, medindo a atividade cerebral de coelhos por meio da técnica de $\mathrm{EEG}^{2}$, chegou a conclusão de que os cérebros destes animais podem utilizar-se de um mecanismo de bifurcação na percepção de odores específicos. Ele demonstrou, experimentalmente, que os sinais de atividade cerebral apresentavam dinâmica caótica, quando os coelhos não sofriam estimulação (nenhum odor específico era inalado), alternando para uma dinâmica próxima de periódica, quando um odor previamente conhecido era inalado. Essa mudança de comportamento pode ser entendida como um fenômeno de bifurcação, causada pela variação de um ou mais "parâmetros" do cérebro do animal.

\section{Diagrama de bifurcações}

Considere o mapa logístico, estudado nas seções anteriores:

$$
x(n+1)=A x(x)(1-x(x)) .
$$

A parametrização dada por $A$ resulta em diferentes tipos de comportamento ou dinâmica, para o sistema em questão. O procedimento geométrico, utilizado para visualizar a natureza dessa variação de parâmetros, consiste do Diagrama de Bifurcação, esboçado numericamente na Figura 2.9. Nesse diagrama, o valor de $A$ no eixo- $x$ é variado em um certo intervalo, e no eixo-y são marcadas as soluções $x(n)$ para $n \rightarrow \infty$. Numericamente, esse diagrama é aproximado plotando-se o estado do sistema após o transiente (intervalo de tempo em que o sistema ainda não se estabeleceu em uma dinâmica específica). Uma linha sólida denota soluções estáveis, e uma linha tracejada indica soluções instáveis nesses diagramas. Em esboços do diagrama por meio de simulações, como apresentado na Figura 2.9, linhas pontilhadas não são desenhadas, pois soluções instáveis não são observadas experimentalmente.

Observa-se pela Figura 2.9, que uma bifurcação ocorre em aproximadamente $A=3.0$, a partir de onde a solução $x(n)$ começa a oscilar entre dois valores (notar a bifurcação da linha). As bifurcações continuam a ocorrer, conforme $A$ é aumentado, dando origem a ciclos periódicos de períodos sempre dobrados até quando o caos aparece, em aproximadamente $A=3.6$. Essa transição ou "rota para o caos" ocorre pelo que se denomina uma rota de

\footnotetext{
${ }^{2}$ Eletroencefalogramo.
} 
duplicação de período. É interessante observar que essa mudança entre comportamentos do sistema é também apreendido pelo cálculo dos expoentes de Lyapunov (compare o diagrama da Figura 2.9 com o da Figura 2.7).

Nota: Uma propriedade interessante do diagrama apresentado na Figura 2.9 é sua estrutura frac$t a l^{3}$ ou auto-similar. Se for aplicado um "zoom” em qualquer pedaço do diagrama, a estrutura resultante vista será similar a do diagrama integral. Essa propriedade expressa bem o conceito de holismo, que afirma que "o todo pode ser encontrado em cada uma de suas partes".

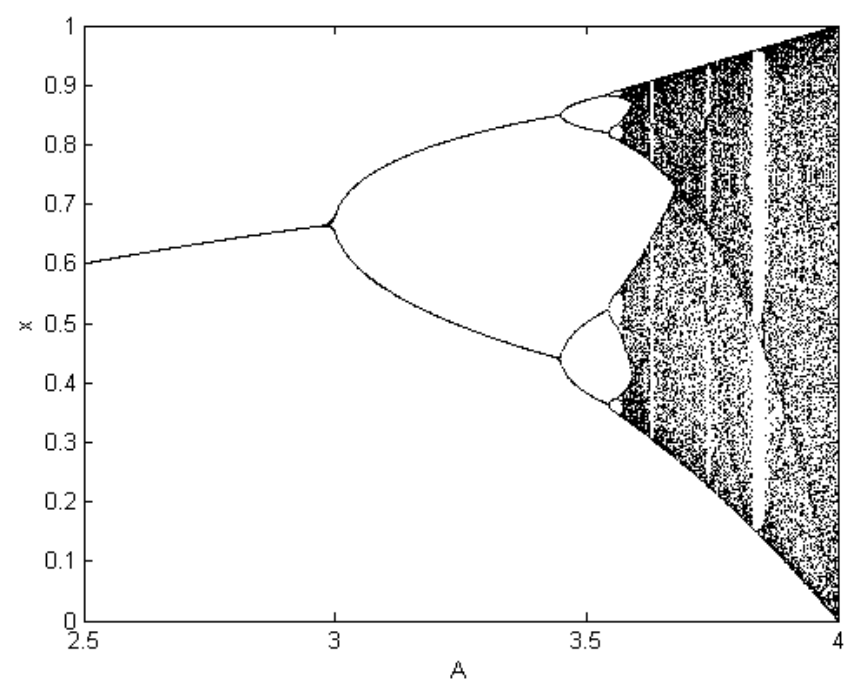

Figura 2.9: Esboço numérico do diagrama de bifurcação de (2.6).

\subsection{Sistemas contínuos n-dimensionais}

Os conceitos de dinâmica, estudados para sistemas discretos unidimensionais, são válidos também para sistemas contínuos e de maiores dimensões. Isto inclui os tipos de dinâmica nãolineares investigadas nas seções anteriores (ponto fixo, periódica, quase-periódica e caótica).

Considere o sistema dinâmico contínuo dado por:

$$
\dot{x}=f(x)
$$

onde $x: I \rightarrow \mathbb{R}^{n}\left(I \subset R\right.$ ) é o estado do sistema e $f: \mathbb{R}^{n} \rightarrow \mathbb{R}^{n}$ é uma função diferenciável, que representa a lei de evolução do sistema. Nesta seção, são considerados sistemas desse tipo, denominados autônomos ou invariantes no tempo, pois a lei $f$ não depende explicitamente do

\footnotetext{
${ }^{3}$ Um fractal é uma figura geométrica que apresenta auto-similaridade invariante à escala em que esta é visualizada.
} 
tempo. Esta exposição se concentrará em sistemas desse tipo, porque eles são mais comuns em modelagens matemáticas e, também, porque são de mais fácil análise. Não serão aqui tratados, portanto, sistemas em que a lei $f$ depende do tempo $(f(t, x))$, ou seja, os sistemas ditos nãoautônomos.

Analogamente ao caso discreto, pode-se esboçar as órbitas de (2.14) mas, para isso, apenas sistemas bidimensionais serão considerados, uma vez que suas órbitas podem ser esboçadas mais facilmente como curvas no plano. Seja, portanto, o sistema autônomo definido por:

$$
\begin{aligned}
& \dot{x}_{1}=f_{1}\left(x_{1}, x_{2}\right) \\
& \dot{x}_{2}=f_{2}\left(x_{1}, x_{2}\right)
\end{aligned}
$$

Nesse caso, as órbitas ou soluções $x(t)=\left(x_{1}(t), x_{2}(t)\right)$ são curvas no plano, como afirma a próxima definição.

Definição 2.5.1 A curva parametrizada por $t$

$$
\left\{\left(x_{1}(t), x_{2}(t)\right) \in \mathbb{R}^{2}: t \in\left(\omega_{-}, \omega_{+}\right)\right\}
$$

é dita uma órbita de (2.15).

A lei de evolução $f$ do sistema autônomo (2.14), pode ser vista como um campo vetorial, que associa a cada ponto $x$ do espaço de estados, um vetor $f(x)$. Para efeito de visualização, considere o sistema (2.15), com $f(x)=\left(2 x_{1},-1 / 2 x_{2}\right)$. A cada ponto $(x, y)$ do plano, é associado um vetor $f(x)=\left(f_{1}(x), f_{2}(x)\right)$ com origem em $x$, como esboça a Figura 2.10.

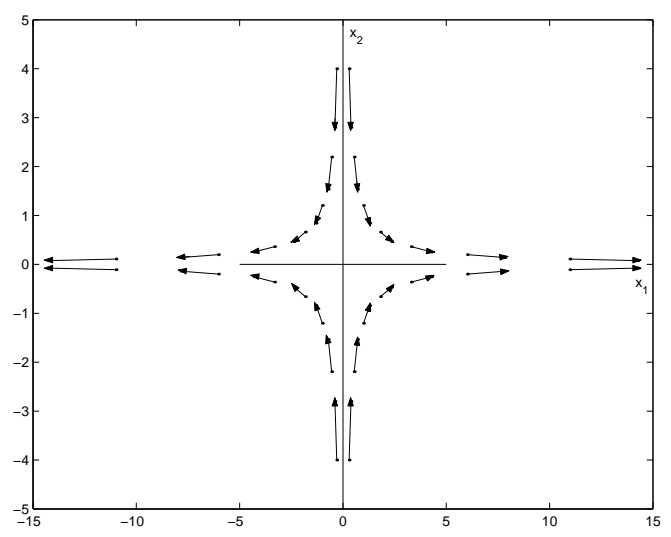

Figura 2.10: Esboço do campo vetorial de (2.15), para $f(x)=\left(2 x_{1},-1 / 2 x_{2}\right)$.

Analogamente a pontos fixos em sistemas discretos, nos sistemas contínuos tem-se pontos de equilíbrio, que são definidos como segue. 
Definição 2.5.2 Um ponto (vetor) $x^{*}$ de (2.14) é um ponto de equilibrio do campo vetorial $f$, se

$$
f\left(x^{*}\right)=0
$$

De maneira análoga ao caso discreto, também são definidas soluções periódicas para sistemas contínuos. No entanto, este texto se restringirá apenas ao estudo de pontos de equilíbrios, uma vez que os resultados obtidos para esse tipo de dinâmica são suficientes para o desenvolvimento da dissertação.

\subsubsection{Sistemas lineares autônomos no plano}

Esta seção trata de sistemas definidos no plano, sendo assim possível visualizar o comportamento das soluções, que são plotadas como curvas no $\mathbb{R}^{2}$. Resultados obtidos com o estudo de sistemas lineares se mostrarão importantes na análise de sistemas não-lineares, como apresentado nas próximas seções.

Considere o sistema (2.14) como sendo linear. Isto é:

$$
\dot{x}=A x
$$

onde $A \in \mathbb{R}^{n \times n}$. Suponha $A$ não-singular ${ }^{4}$ (esta hipótese será relaxada mais adiante no texto). Pode-se diagonalizar $A$, colocando o sistema (2.16) em uma forma mais simples, por uma mudança de coordenadas em que esse é definido. Seja $S=\left[v_{1}, v_{2}, \ldots, v_{n}\right] \in \mathbb{R}^{n \times n}$ a matriz que realiza essa mudança de coordenadas, onde $v_{i}, i=1, \ldots, n$ são os autovetores de $A$, e seja $\Lambda$ a matriz diagonal formada pelos autovalores $\lambda_{1}, \ldots, \lambda_{n}$ de $A$. É possível mostrar [Lay, 1996] que $A$ pode ser fatorada como

$$
A=S \Lambda S^{-1}
$$

Assim, a seguinte mudança de variáveis pode ser realizada

$$
y=S^{-1} x \Rightarrow x=S y
$$

onde $y \in \mathbb{R}^{n}$ representa o estado do sistema (2.16) nessa nova base, desde que $S$ seja nãosingular (isto é, os autovetores de $A$ devem ser linearmente independentes). Derivando ambos os lados da igualdade anterior, obtém-se

\footnotetext{
${ }^{4} A$ é invertível ou $\operatorname{det}(A) \neq 0$ ou os autovalores de $A$ são não-nulos.
} 


$$
\dot{y}=S^{-1} \dot{x}=S^{-1} A x=S^{-1}\left(S \Lambda S^{-1}\right) x=\Lambda S^{-1}(S y)=\Lambda y
$$

Logo, o sistema (2.16) nessa nova base é:

$$
\dot{y}=\Lambda y
$$

onde $\Lambda=\operatorname{diag}\left(\lambda_{1}, \ldots, \lambda_{n}\right)$. Ou seja, tem-se um sistema da forma:

$$
\left[\begin{array}{c}
\dot{y}_{1} \\
\dot{y}_{2} \\
\vdots \\
\dot{y}_{n}
\end{array}\right]=\left[\begin{array}{cccc}
\lambda_{1} & 0 & \cdots & 0 \\
0 & \lambda_{2} & & \vdots \\
\vdots & & \ldots & 0 \\
0 & \cdots & 0 & \lambda_{n}
\end{array}\right] \cdot\left[\begin{array}{c}
y_{1} \\
y_{2} \\
\vdots \\
y_{n}
\end{array}\right]
$$

onde cada equação está "desacoplada" da outra (isto é, cada equação pode ser resolvida independentemente da outra). Uma função $y(t)$, que seja solução não-trivial de (2.18), é dada por

$$
y(t)=\left[\begin{array}{c}
c_{1} e^{\lambda_{1} t} \\
c_{2} e^{\lambda_{2} t} \\
\vdots \\
c_{n} e^{\lambda_{n} t}
\end{array}\right]
$$

onde $\left[c_{1}, \ldots, c_{n}\right]^{T}=y(0)=S^{-1} x(0)=S^{-1} x_{0}$ são constantes determinadas pelas condições iniciais $x(0)=x_{0}$ do sistema. A solução geral para (2.16), na base ou coordenadas originais, podem ser obtidas da equação anterior, substituindo $x(t)=S y(t)$ :

$$
x(t)=\left[v 1, \ldots, v_{n}\right] \cdot y(t)=c_{1} v_{1} e^{\lambda_{1} t}+\ldots+c_{n} v_{n} e^{\lambda_{n} t}
$$

Para o caso mais genérico em que $A$ possa ser singular em (2.16), utiliza-se a fatoração de A denominada Forma Canônica de Jordan (ver Lema A.0.1 no Apêndice A). Por meio dessa fatoração, obtém-se

$$
A=M J M^{-1}
$$

A solução geral de (2.16) é, então

$$
x(t)=M e^{J} x_{0}
$$

onde $x_{0}$ é a condição inicial e a função $e^{J}$ é o exponencial ${ }^{5}$ do operador $J$.

${ }^{5} \mathrm{O}$ exponencial de $J$ é definido como $e^{J}=\sum_{k=0}^{\infty} \frac{J^{k}}{k !}$, onde $k$ ! denota o fatorial de $k$ e $0 !=1$, por definição. 


\section{Comportamento qualitativo das soluções}

Para se estudar o comportamento qualitativo das soluções (2.20) do sistema linear (2.16), considere o caso bidimensional, isto é:

$$
\dot{x}=A x
$$

onde $x=\left[x_{1}, x_{2}\right]^{T} \in \mathbb{R}^{2}$ e $A \in \mathbb{R}^{2 \times 2}$. Dependendo dos autovalores de $A$, a Forma Canônica de Jordan $J$ em (2.20) pode assumir uma das seguintes formas:

$$
\left[\begin{array}{ll}
\lambda_{1} & 0 \\
0 & \lambda_{2}
\end{array}\right],\left[\begin{array}{ll}
\lambda & k \\
0 & \lambda
\end{array}\right],\left[\begin{array}{ll}
\alpha & -\beta \\
\beta & \alpha
\end{array}\right]
$$

onde $k$ é 0 ou 1. A primeira forma corresponde a autovalores $\lambda_{1}$ e $\lambda_{2}$ reais e distintos, a segunda forma a autovalores reais e iguais e a terceira a autovalores complexos $\lambda_{1,2}=\alpha \pm i \beta$. Assim, todos os possíveis comportamentos da solução (2.20) podem ser classificados de acordo com os autovalores de $A$, em [Khalil, 1996]:

Caso 1: autovalores reais e distintos. Tem-se, então, os seguintes comportamentos:

(a) Nó estável: $\lambda_{1}<\lambda_{2}<0$. Nesse caso, ambos autovalores são reais e negativos, mas um deles é maior que o outro. Isto causa um decaimento exponencial mais acentuado em uma das direções (eixos) do sistema (àquele que corresponde a $\lambda_{1}$ ) (ver Figura 2.11(a));

(b) Nó instável: $\lambda_{1}>\lambda_{2}>0$. Tem-se uma expansão, que se propaga mais rapidamente em uma das direções (eixo associado a $\lambda_{1}$ ) (ver Figura 2.11(b));

(c) Sela: $\lambda_{1}<0<\lambda_{2}$. Tem-se uma contração, devida a $\lambda_{1}$, e uma expansão, devida a $\lambda_{2}$ (ver Figura 2.11(c)).

Caso 2: autovalores reais e iguais. Tem-se duas opções de comportamento:

(a) Nó impróprio estável: $\lambda_{1}=\lambda_{2}<0$. Ocorrem contrações com a mesma intensidade em ambas direções. Corresponde à segunda matriz em (2.22), onde $k=0$ ou $k=1$ (ver Figuras 2.12(a) e 2.12(b));

(b) Nó impróprio instável: $\lambda_{1}=\lambda_{2}>0$. Nesse caso ocorrem expansões com igual intensidade em ambas as direções do sistema. Corresponde ao segundo tipo de matriz em (2.22), onde $k=0$ ou $k=1$ (ver Figuras 2.12(c) e 2.12(d)).

Caso 3: autovalores complexos. Distinguem-se três tipos de comportamento:

(a) Foco estável: $\lambda=\alpha \pm i \beta$, com $\alpha<0$ e $\beta \neq 0$. As soluções se encaminham, girando, para a origem (ver Figura 2.13(a)); 


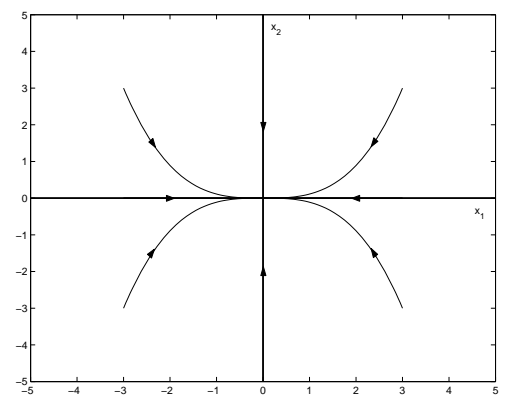

(a) Nó estável

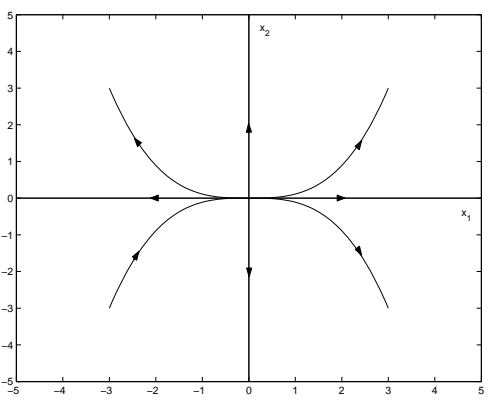

(b) Nó instável

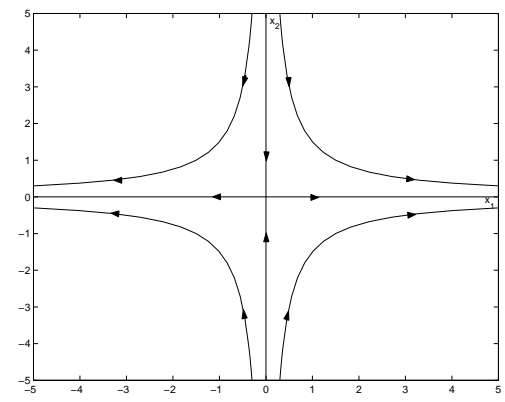

(c) Sela

Figura 2.11: Caso 1 - autovalores reais e distintos.

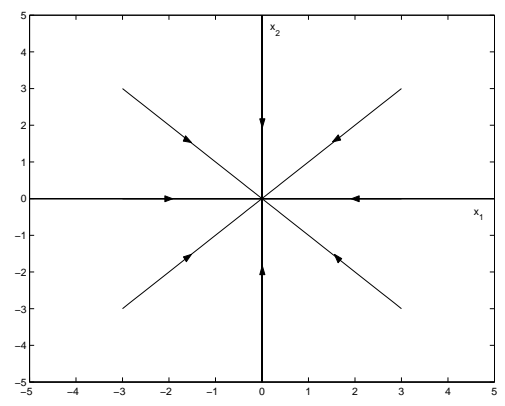

(a) No impróprio estável, $k=0$

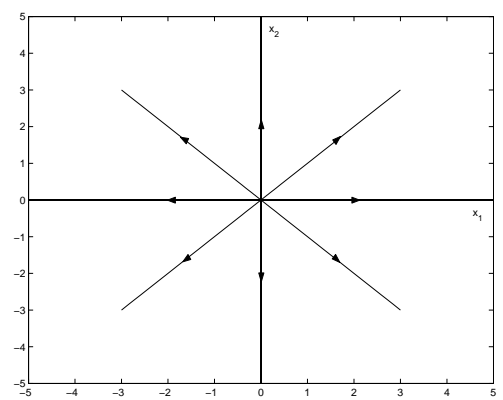

(c) Nó impróprio instável, $k=0$

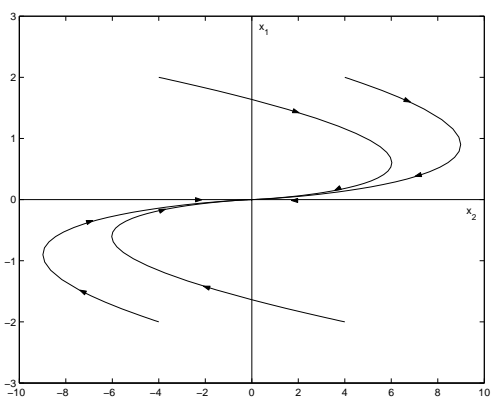

(b) Nó impróprio estável, $k=1$

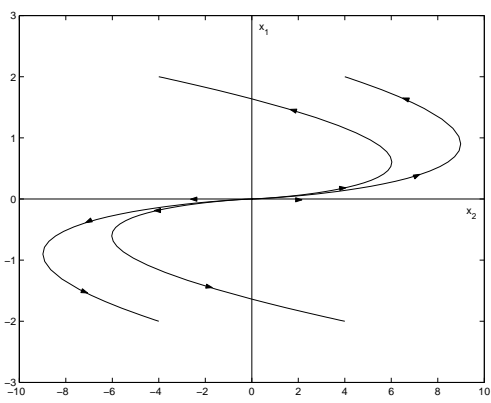

(d) Nó impróprio instável, $k=1$

Figura 2.12: Caso 2 - autovalores reais e iguais.

(b) Foco instável: $\lambda=\alpha \pm i \beta$, com $\alpha>0$ e $\beta \neq 0$. As soluções se expandem em espiral, afastando-se da origem (ver Figura 2.13(b)); 
(c) Centro: $\lambda= \pm i \beta, \beta \neq 0$. As órbitas são curvas fechadas em torno da origem (ver Figura 2.13(c)). Para $\beta>0$, o sentido do percurso é anti-horário.

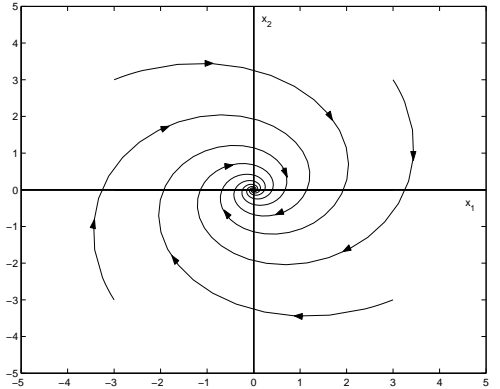

(a) Foco estável

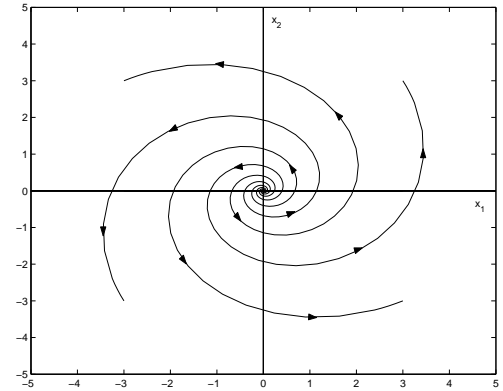

(b) Foco instável

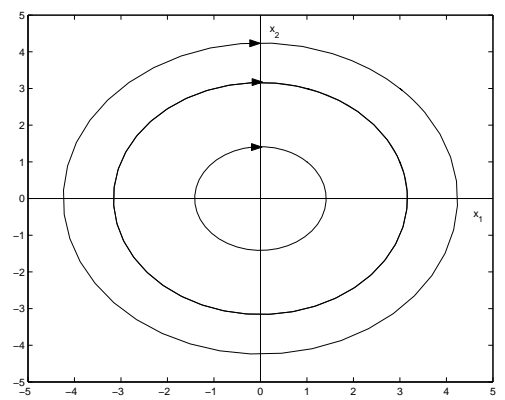

(c) Centro

Figura 2.13: Caso 3 - autovalores complexos.

As Figuras 2.11(a)-2.13(c) ilustram as órbitas do sistema (2.21), em cada um dos casos citados. A este tipo de ilustração das órbitas, dá-se o nome de retrato de fase do sistema.

\subsubsection{Estabilidade de sistemas lineares}

As soluções para (2.21), estudadas qualitativamente na seção anterior, fornecem informação acerca da estabilidade de pontos de equilíbrio, apenas observando-se os autovalores de $A$. Este critério é usado na determinação da estabilidade do sistema linear (2.16), como afirma o próximo teorema.

Teorema 2.5.3 O ponto de equilíbrio $x=0$ em (2.16) é estável se, e somente se, todos autovalores de A satisfazem Re $\lambda_{i} \leq 0$, e todo autovalor com Re $\lambda_{i} \leq 0$ tem um bloco de Jordan de ordem um. O ponto de equilíbrio $x=0$ é assintoticamente estável se, e somente se, todos autovalores de A satisfazem $\operatorname{Re} \lambda_{i} \leq 0$.

Prova: A prova desse teorema está além do escopo deste texto, podendo ser encontrada em [Khalil, 1996]. 
Na próxima seção, é analisada a estabilidade de pontos de equilíbrio em sistemas nãolineares mas, antes disso, considere uma definição mais formal de estabilidade (comparar com as definições dadas na Seção 2.4.1) em termos de epsilons e deltas $(\epsilon, \delta)$.

Definição 2.5.4 Seja $x^{*}$ um ponto de equilíbrio de (2.14).

(a) O ponto de equilíbrio $x^{*}$ é estável (no sentido de Lyapunov) se, dados $\epsilon>0$ e $t_{0} \in[0, \infty)$, existe um $\delta\left(\epsilon, t_{0}\right)>0$, tal que $\left\|x\left(t_{0}\right)-x^{*}\right\|<\delta$ implica $\left\|f^{n}\left(x\left(t_{0}\right)\right)-x^{*}\right\|<\epsilon$, para todo $t>t_{0}$

(b) O ponto de equilíbrio $x^{*}$ é dito assintoticamente estável se, além de ser estável, existir um $\eta\left(t_{0}\right)>0$ tal que

$$
\left\|x\left(t_{0}\right)-x^{*}\right\|<\eta \quad \text { implica } \quad \lim _{t \rightarrow \infty} x(t)=x^{*}
$$

(c) O ponto de equilíbrio $x^{*}$ é dito instável se ele não for estável.

\subsubsection{Linearização ou Método Indireto de Lyapunov}

Considere o sistema não-linear:

$$
\dot{x}=f(x)
$$

e suponha $f$ continuamente diferenciável. Seja $x^{*}$ um ponto de equilíbrio de (2.23). O lado direito da equação anterior pode ser expandido em série de Taylor, em torno desse ponto $x^{*}$ :

$$
f(x)=f\left(x^{*}\right)+D f\left(x^{*}\right)\left(x-x^{*}\right)+g(x)
$$

onde $D f\left(x^{*}\right)$ é a matriz Jacobiana, que contém as derivadas parciais de $f$ calculadas no ponto $x^{*}$ :

$$
D f\left(x^{*}\right)=\left.\frac{\partial f(x)}{\partial x}\right|_{x=x^{*}}
$$

e onde $g(x)=O\left(x^{2}\right)$ representa os termos de ordem superior da expansão. Como $x^{*}$ é um ponto de equilíbrio, então (ver Definição 2.5.2):

$$
f\left(x^{*}\right)=0
$$

e a equação (2.24) fica: 


$$
f(x)=D f\left(x^{*}\right)\left(x-x^{*}\right)+g(x)
$$

Logo, (2.23) pode ser reescrita como:

$$
\dot{x}=D f\left(x^{*}\right)\left(x-x^{*}\right)+g(x) .
$$

Fazendo-se a seguinte mudança de variáveis

$$
y=x-x^{*}
$$

e derivando ambos os lados, tem-se

$$
\dot{y}=\dot{x}
$$

A partir dessa mudança de variáveis, obtém-se o seguinte sistema não-linear equivalente à (2.23):

$$
\dot{y}=D f\left(x^{*}\right) y+g\left(y+x^{*}\right)
$$

cujo ponto de equilíbrio considerado é a origem do sistema. Esta forma de (2.23) é utilizada para facilitar a análise em resultados futuros.

Considerando-se o comportamento de soluções de (2.26) (ou, equivalentemente, de (2.23)) apenas em uma pequena vizinhança em torno do equilíbrio, pode-se obter o seguinte sistema linear associado à (2.26), desprezando-se os termos de ordem superior $g(x)$ da expansão:

$$
\begin{aligned}
& \dot{y}=D f\left(x^{*}\right) y \\
& \dot{y}=A y
\end{aligned}
$$

É possível mostrar que as órbitas do sistema não-linear (2.26) (ou, equivalentemente, (2.23)) se comportam de maneira semelhante às órbitas do sistema linear associado (2.27), em uma vizinhança próxima da origem [Hartman, 1964]. Assim, a estabilidade de pontos de equilíbrio de (2.23) pode ser verificada, como afirma o próximo teorema.

Teorema 2.5.5 Seja a origem $x=0$ um ponto de equilíbrio do sistema

$$
\dot{x}=f(x)
$$

onde $f: D \rightarrow \mathbb{R}^{n}$ é continuamente diferenciável em uma vizinhança $D$ da origem. Considere a matriz 


$$
A=\left.\frac{\partial f}{\partial x}(x)\right|_{x=0}
$$

Pode-se afirmar que:

1. A origem é assintoticamente estável, se todos autovalores de A tiverem parte real negativa $\left(\operatorname{Re} \lambda_{i}<0\right.$, para todo $\left.i\right)$.

2. A origem é instável, se um ou mais autovalores de A tiverem parte real positiva (Re $\lambda_{i}>$ 0 , para algum i).

Prova: A prova desse teorema está além do escopo deste texto, podendo ser encontrada em [Khalil, 1996].

Observação 2.5.6 Não há perda de generalidade em se considerar o ponto de equilíbrio do Teorema 2.5.5 como sendo a origem. Por uma mudança de variáveis (como feito em (2.25)), é possível deslocar um ponto de equilíbrio qualquer para a origem e aplicar, assim, os resultados apresentados pelo teorema.

Observação 2.5.7 O Teorema 2.5.5 nada afirma sobre autovalores de A com parte real nula ( Re $\left.\lambda_{i}=0\right)$. Nesse caso, o método falha em determinar a estabilidade do ponto de equilíbrio.

De acordo com o teorema anterior, é possível determinar a estabilidade de pontos de equilíbrio de um sistema não-linear em termos da matriz Jacobiana de $f$, da mesma forma como se faz para um sistema linear (compare os resultados dos Teoremas 2.5.5 e 2.5.3). No caso de $f$ ser não-linear em (2.23), esse resultado afirma que uma linearização de $f$ em torno da origem, permite tirar conclusões a respeito da estabilidade dos pontos de equilíbrio do sistema. Por esse motivo, esse resultado é conhecido como método de linearização.

\subsubsection{Segundo Método ou Método Direto de Lyapunov}

A estabilidade de um ponto de equilíbrio do sistema não-linear (2.23) pode ser analisada observando a parte linear de $f$, como visto na seção anterior. No entanto, quando os autovalores da parte linear são nulos, por exemplo, nada se pode concluir com aquele método, como afirma a Observação 2.5.7. Assim, em 1892, o engenheiro e matemático russo A. M. Lyapunov encontrou outro critério de estabilidade, que permite generalizar a noção de norma em $\mathbb{R}^{n}$. Ele mostrou que outras funções além da norma podem ser usadas para verificar quando $\left|x(t)-x^{*}\right|$ decresce, considerando-se $x(t)$ uma solução próxima do ponto de equilíbrio $x^{*}$. Essas funções receberam o nome de funções de Lyapunov. 
Considere o sistema autônomo

$$
\dot{x}=f(x)
$$

onde $f: U \rightarrow \mathbb{R}^{n}$ é uma função continuamente diferenciável ( $U \subset \mathbb{R}^{n}$ é um subconjunto aberto $^{6}$ contendo a origem) tal que $f(0)=0$ e tal que a origem é um equilíbrio isolado, ou seja, existe uma vizinhança $N_{0}$ da origem onde $f(x) \neq 0, \forall x \in N_{0}, x \neq 0$.

Seja $V: U \rightarrow \mathbb{R}^{n}$ uma função diferenciável. A derivada

$$
\dot{V}(x)=\nabla V(x) \cdot \dot{x}
$$

definida para cada $x \in U$, é chamada derivada de $V$ ao longo das soluções de (2.28), onde $\nabla V$ denota o gradiente de $V$. A próxima definição introduz o conceito de função definida positiva, que será utilizada no teorema seguinte.

Definição 2.5.8 Uma função $V: U \rightarrow \mathbb{R}$ é dita definida positiva se

$$
V(x)>0
$$

para todo $x \in U, x \neq 0$.

A partir desses fatos, pode-se enunciar o Teorema de Lyapunov.

Teorema 2.5.9 (Teorema de Lyapunov) Seja $x=0$ um ponto de equilíbrio do sistema (2.28). Se existir uma função definida positiva $V(x)$, em uma vizinhança $U$ da origem, tal que $\dot{V}(x) \leq 0$, para $x \in U$, então o ponto de equilíbrio $x=0$ é estável. Além disso, se $\dot{V}(x)$ for definida negativa em uma vizinhança $U$ da origem, então o ponto de equilíbrio $x=0$ é assintoticamente estável.

Prova: A prova do teorema está além do escopo deste texto, podendo ser encontrada em [Ladeira, 2001], [Khalil, 1996].

A função $V(x)$, mencionada no teorema anterior, recebe o nome de função de Lyapunov.

Observação 2.5.10 O Método Direto de Lyapunov, que consiste do Teorema 2.5.9, afirma a existência de funções de Lyapunov $V$, mas não dá pistas de como encontrá-las. Ele apresenta apenas uma condição suficiente, mas não necessária, para determinar a estabilidade de um ponto de equilíbrio. Isso quer dizer que a incapacidade de se encontrar uma função $V(x)$, tal qual afirma o teorema, não indica que o sistema analisado seja instável, ou que seja apenas estável, e não assintoticamente estável. Apenas, nesse caso, não se pode obter maiores informações sobre a estabilidade do sistema, utilizando tal método.

\footnotetext{
${ }^{6}$ Um subconjunto $S \subset \mathbb{R}^{n}$ é dito aberto se, para cada vetor $x \in S$, é possível encontrar uma vizinhança de $x$ com raio $\epsilon: N(x, \epsilon)=\left\{z \in \mathbb{R}^{n}:\|z-x\|<z\right\}$ tal que $N(x, \epsilon) \subset S$.
} 
Uma função de Lyapunov também pode ser vista como uma generalização do conceito de função de energia de um sistema mecânico. Se o sistema for dissipativo, a têndencia é a energia deste sempre diminuir (isto corresponde a $\dot{V} \leq 0$ ). Em aplicações desse método a sistemas reais, uma pista é utilizar a função de energia do sistema como candidata à função de Lyapunov do mesmo. Caso isto não seja possível, existem outros meios de se obter uma função tal qual afirma o teorema. Como exemplo, na próxima seção, é apresentado um método com esse objetivo. Este método será útil, posteriormente, na determinação de "funções de energia" para Redes Neurais Artificiais.

\section{Método do gradiente variável}

Em 1962, D.G. Schultz propôs um procedimento sistemático para encontrais funções $V$ como afirma o Teorema 2.5.9. Seja $V: \mathbb{R}^{n} \rightarrow R$ uma função diferenciável. A derivada de $V$, ao longo das trajetórias de (2.28), é dada por:

$$
\dot{V}(x)=\nabla V(x) \cdot f(x)=<g(x), f(x)>
$$

onde $g: \mathbb{R}^{n} \rightarrow \mathbb{R}^{n}, g(x)=\nabla V(x)$ e onde $<g(x), f(x)>$ denota o produto interno usual. Como $f(x)$ é conhecida (representa a lei de evolução do sistema), pode-se escolher a função $g(x)$ de modo que se obtenha uma função $V(x)$ definida positiva e $\dot{V}(x)$ definida negativa, de acordo com o Teorema de Lyapunov (Teorema 2.5.9). Essa escolha, no entanto, deve estar em conformidade com a proposição seguinte.

Proposição 2.5.11 Seja $g: \mathbb{R}^{n} \rightarrow \mathbb{R}^{n}$ diferenciável e $V: \mathbb{R}^{n} \rightarrow \mathbb{R}$. A função $g(x)$ é o gradiente da função escalar $V(x)$ (isto é, $g(x)=\nabla V(x)$ ) se, e somente se,

$$
\frac{\partial g_{i}}{\partial x_{j}}=\frac{\partial g_{j}}{\partial x_{i}}, \quad \forall i, j=1, \ldots, n
$$

Prova: A prova dessa proposição esta além do escopo deste texto ${ }^{7}$.

A proposição anterior afirma que a matriz Jacobiana de $g(x)$ deve ser simétrica. Dada esta restrição, a função $g$ é escolhida tal que $<g(x), f(x)>$ seja definida negativa. A função $V(x)$ é então obtida integrando $g(x)=\left(g_{1}(x), g_{2}(x), \ldots, g_{n}(x)\right)$ :

$$
V(x)=\int_{o}^{x} g^{T}(\eta) d \eta=\int_{0}^{x} \sum_{i=1}^{n} g_{i}(\eta) d \eta_{i}
$$

\footnotetext{
${ }^{7}$ Demonstramos esta proposição para 2 dimensões, usando os Teoremas de Schwarz, de Green e de Existência de Função Potencial[Guidorizzi, 2002]. No entanto, para estender tal demonstração para o caso de $n$ dimensões, faz-se necessário o uso do Teorema de Stokes, que é uma generalização do Teorema de Green no $\mathbb{R}^{n}$, mas que exige a utilização de estruturas denominadas k-formas, o que está além do escopo dessa dissertação.
} 
Como $g(x)$ define um campo vetorial conservativo $(g(x)=\nabla V)$, a integral acima é independente do caminho de integração. Alguns parâmetros de $g(x)$ devem ser também escolhidos para que a função $V(x)$, obtida acima, seja definida positiva.

No Capítulo 4, é ilustrado como o Método do Gradiente Variável pode ser utilizado para encontrar uma função de energia para o modelo de Hopfield.

\subsection{Conclusão}

Sistemas dinâmicos têm sido estudados como modelos de fenômenos que evoluem no tempo, nas mais diversas áreas da ciência. Constituem uma forma de se conhecer, prever, ou mesmo de se controlar o comportamento de sistemas modelados por equações diferenciais ou equações de diferenças. Particular atenção foi dada nesse capítulo ao estudo de sistemas não-lineares, muito comuns na Natureza, mas de difícil análise, devido à sua intrínseca complexidade. Outra classe de sistemas dinâmicos também muito estudados são aqueles de alta dimensionalidade, como, por exemplo, os modelos RNAs que são apresentados nos capítulos subseqüentes.

Basicamente, neste capítulo, foram revistos os principais conceitos de dinâmica, sendo que aspectos relevantes sobre estabilidade também foram apresentados. Para sistemas contínuos, foram discutidos resultados de estabilidade como o Método Direto de Lyapunov, que será útil na análise da estabilidade das RNAs investigadas nessa dissertação.

O próximo capítulo apresentada uma introdução ao estudo de RNAs, sendo que maior atenção será dada às redes neurais realimentadas, que são modeladas como sistemas dinâmicos não-lineares. 


\section{Capítulo 3}

\section{Redes Neurais Artificiais}

O estudo do cérebro é atrativo, sob o ponto de vista da Computação, por propiciar o desenvolvimento de modelos de processamento de informação biologicamente inspirados, como é o caso das Redes Neurais Artificiais (RNAs). As RNAs representam uma tentativa de superar limitações que o computador digital apresenta, buscando, para isso, imitar os princípios de funcionamento do cérebro. As principais característica do cérebro, se visto como um dispositivo de computação, são: alto grau de paralelismo; operação mesmo sob sinais corrompidos por ruído; robustez e tolerância à falhas, graças à redundância e operação descentralizada dos neurônios; capacidade de adaptação e auto-organização, conseguidos com a experiência e/ou aprendizado. De um ponto de vista computacional, o cérebro é um computador analógico ${ }^{1}$ que, ao contrário de computadores digitais que processam símbolos, utiliza sinais de origem eletro-química para efetuar computações.

Muitas simplificações se fazem necessárias para que se obtenha um modelo do cérebro, as quais dependem da finalidade a que se destina tal modelo. Existem, por exemplo, modelos de neurônio extremamente detalhados, como é o caso do modelo de Hodgin e Huxley [Hodgkin \& Huxley, 1952], utilizados em pesquisas em Neurociência. Este modelo descreve, por meio de equações diferencias ordinárias (EDOs), a dinâmica de geração de potenciais de ação na membrana do neurônio. No entanto, sua utilização para a investigação do processamento de informação no cérebro se mostra inviável, já que uma rede formada por tais neurônios se torna muita complexa para ser analisada, ou mesmo simulada. No entanto, o estudo de RNAs, sob o ponto de vista da computação, se utiliza de modelos mais simples e, portanto, mais adequados para fins de investigação da capacidade computacional do cérebro, principalmente quanto à resolução de problemas ou aplicações reais. Na próxima seção, é apresentado o princípio de funcionamento do neurônio biológico.

\footnotetext{
${ }^{1}$ Um computador analógico faz uma analogia direta a um meio físico para realizar uma operação matemática ou computação. Pode utilizar, por exemplo, níveis contínuos de diferença de potencial em um circuito, para representar valores sendo computados.
} 


\subsection{Princípio de funcionamento do neurônio}

De forma geral, o comportamento de um neurônio depende do estado em que ele se encontra, e do grau de estimulação que recebe. Estímulos provenientes de outros neurônios, chegam como um sinal eletro-químico até a membrana de uma célula nervosa, através dos dendritos (ver Figura 3.1). Se estes estímulos atingirem um certo limiar (threshold), a célula pode disparar um potencial de ação, que são descargas elétricas geradas no prolongamento da célula denominado axônio. Estes axônios, que se encaminham até a outra extremidade do neurônio, se ramificam para estabelecer comunicação com células vizinhas. Tais ramificações, que se encontram normalmente muito próximas, não estão exatamente em contato com as outras células, e esse espaço que as separam é denominado de sinapse. Nas sinapses, o estímulo elétrico que percorre o neurônio, se transforma em um estímulo químico, por meio da liberação de substâncias neurotransmissoras pelo neurônio. Estas substâncias, por sua vez, atuam na estimulação dos dendritos de outras células nervosas as quais o neurônio considerado possui sinapses, propagando, dessa forma, o sinal recebido.

Um modelo de neurônio utilizado em RNAs, entretanto, abstrairá todos esses detalhes intrínsecos de como se procede o fenômeno biológico, como os detalhes da descarga de potenciais de ação, o período refratário (período em que o neurônio não dispara, após ser estimulado), os atrasos na transmissão de sinais intra-neuronais devido à condições específicas do meio, entre outros. Na seção seguinte, é apresentado um modelo clássico de neurônio artificial.

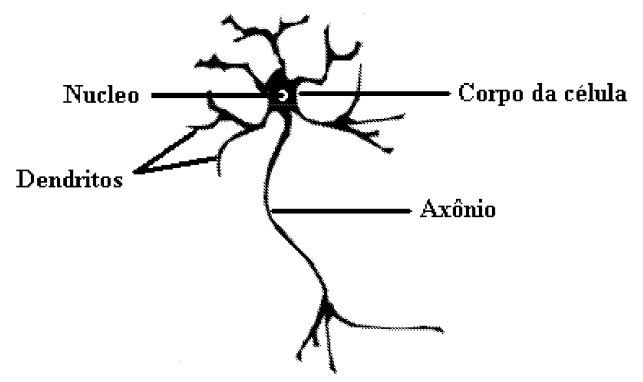

Figura 3.1: Representação de um neurônio.

\subsection{Modelo de neurônio de McCulloch e Pitts}

De maneira simplificada, o chamado modelo de McCulloch e Pitts [McCulloch \& Pitts, 1943] considera um neurônio como sendo um elemento que efetua uma soma ponderada de sinais e que produz uma saída, de acordo com uma função de ativação. Embora um neurônio real emita pulsos de potencial de ação, na forma de um sinal contínuo, no modelo considerado esta informação é simplificada, sendo que o estado do neurônio é 
considerado binário, indicando apenas se ele está disparando potenciais de ação ou não. Experimentalmente, observa-se que existe uma relação entre a quantidade média de estímulos que um neurônio recebe e a quantidade média de potenciais de ação que ele produz, sendo que essa relação toma uma forma sigmoidal ${ }^{2}$ (Figura 3.2(a)). Outras funções de ativação, que são normalmente utilizadas em modelos de neurônio, também possuem uma forma semelhante a esta, e podem ser vizualizadas nas Figuras 3.2.

Matematicamente, o modelo de McCulloch e Pitts é expresso pela seguinte equação de diferenças:

$$
x_{i}(n+1)=f\left(\sum_{j}^{n} w_{i j} x_{j}(n)-\theta_{i}\right), \quad i=1, \ldots, n
$$

onde $x_{i}: \mathbb{Z} \rightarrow B,(B=\{-1,1\})$ representa o estado do $i$-ésimo neurônio $(i=1, \ldots, n)$, que pode estar disparando $\left(x_{i}=1\right)$ potenciais de ação ou pode estar em repouso $\left(x_{i}=-1\right)$. A variável independente $n \in \mathbb{Z}$ indica o tempo, sendo este um valor discreto. A eficiência da conexão entre o $i$-ésimo e o $j$-ésimo neurônio é dada por $w_{i j} \in \mathbb{R}$, para $i, j=1, \ldots, n$. No modelo original, a função de ativação $f$ é a função sinal, cujo gráfico é dado na Figura 3.2(b)). O limiar de operação (threshold) do neurônio é $\theta_{i} \in \mathbb{R}$.

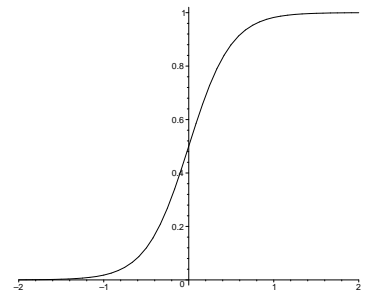

(a) Função Sigmoidal

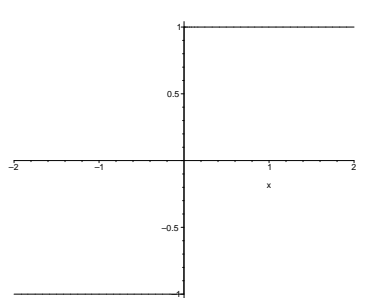

(b) Função Sinal

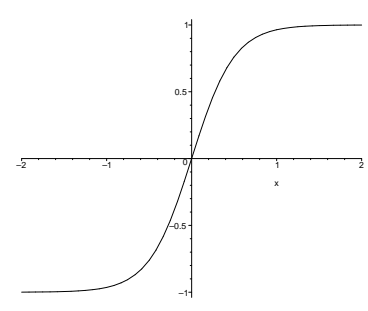

(c) Tangente Hiperbólica

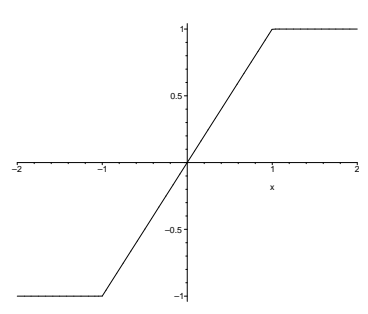

(d) Função Saturação

Figura 3.2: Exemplos de funções de ativação.

\subsection{O paradigma de Computação Neural}

Modelos de RNAs, em geral, tentar apreender a essência do processamento de informação como se supõe que este ocorra no cérebro. Eles definem, por assim dizer, um paradigma neural de computação, que difere do paradigma convencional existente, nos seguintes aspectos:

- os elementos processadores (neurônios) são lentos e pouco confiáveis, se comparados aos componentes eletrônicos de um computador digital;

- a operação da rede é descentralizada, isto é, não existe um elemento central (CPU) que controle o sistema, como ocorre em um computador digital;

\footnotetext{
${ }^{2}$ Uma função sigmoidal é uma função limitada, monotônicamente crescente.
} 
- em RNAs não existe a noção de programa como uma seqüência ordenada, e bem definida, de instruções de máquina. É comum uma rede ou máquina neural "aprender" a tarefa que deve executar.

Mais especificamente, a programação de uma RNA consiste da determinação de:

- conjuntos de exemplos de treinamento, isto é, exemplos de sinais ou padrões que a rede utilizará para aprender;

- a forma da função de ativação dos elementos processadores ou neurônios artificiais;

- a topologia ou arquitetura da rede, isto é, a forma como os neurônios serão interconectados;

- o tipo de algoritmo de aprendizado que se utilizará para treinar a rede. Estes podem ser:

(a) supervisionados, se os exemplos de treinamento são pares do tipo estímulo/resposta esperada;

(b) não-supervisionados, se os exemplos de treinamento consistem apenas de sinais de entrada. Nesse tipo de aprendizado, espera-se que a própria rede encontre correlações entre os estímulo e que, assim, produza uma resposta adequada.

\subsection{Modelos de Redes Neurais}

Com relação aos tipos de modelos matemáticos empregados na descrição de RNAs, estes podem ser classificados em duas categorias:

(a) Modelos Estáticos: a rede é vista como uma função (no sentido de objeto matemático), isto é, sua operação é estabelecer uma relação estímulo-resposta de acordo com a entrada da rede. Nesse caso, os elementos processadores são conectados em uma só direção, como ilustra a Figura 3.3. Essas redes são denominadas Redes Feedforward, ou de alimentação adiante. Exemplos de modelos estáticos de redes neurais são:

- Percetron [Rosenblatt, 1958];

- Perceptron de múltiplas camadas (Multi-Layer Perceptron) [Rumelhart \& McClelland, 1986];

- Mapas auto-organizáveis (SOM - Self Organizing Maps) [Kohonen, 1997];

- ART (Adaptive Resonance Theory) [Carpenter \& Grossberg, 1995]. 
(b) Modelos Dinâmicos: a rede é vista como um sistema dinâmico, isto é, sua operação não é apenas dada em função da entrada, mas também do estado em que a rede se encontra. Dessa forma, diz-se que tais redes possuem memória. Redes neurais nessa categoria possuem realimentações (feedback), como mostra a Figura 3.4, sendo assim denominadas redes recorrentes, dinâmicas ou realimentadas. Alguns exemplos de modelos nessa classe são:

- Modelo de Hopfield [Hopfield, 1982];

- Redes Neurais Celulares (RNCs) [Chua \& Yang, 1988];

- Redes ou reticulados (lattices) de mapas acoplados [Kaneko, 1993];

- Osciladores acoplados [Mirollo \& Strogatz, 1990].

Devido a essa pesquisa se concentrar em modelos do segundo tipo, apenas a classe de modelos dinâmicos será explorada nos capítulos que seguem. A seguir, é apresentado um breve histórico que resume os principais eventos que nortearam as pesquisas com RNAs, nas últimas décadas.

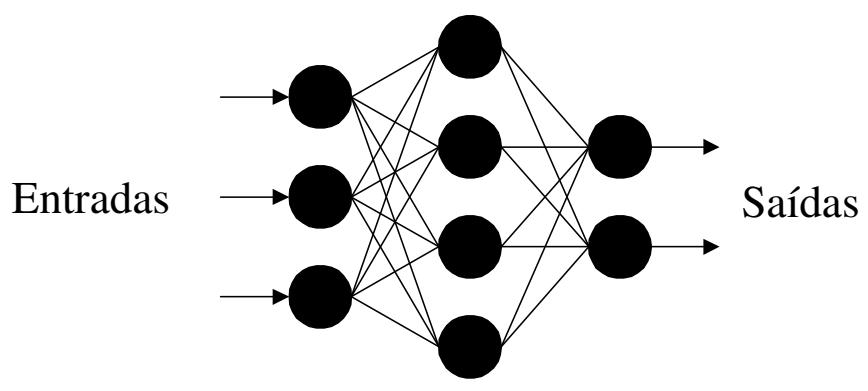

Figura 3.3: Esquema de rede tipo feedforward.

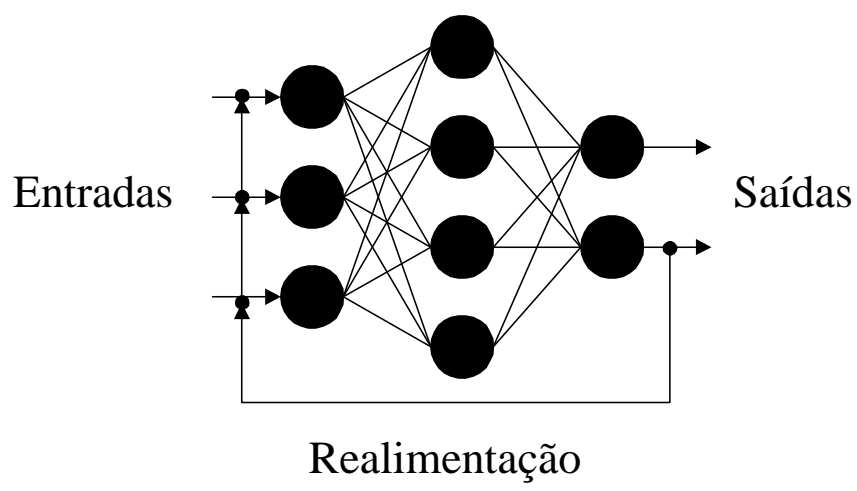

Figura 3.4: Esquema de rede tipo realimentada. 


\subsection{Histórico de pesquisas na área}

Segue um resumo cronológico, não exaustivo, dos resultados mais significativos da pesquisa em RNAs:

1943: Warren McCulloch e Walter Pitts [McCulloch \& Pitts, 1943] propõem um modelo de neurônio artificial e mostram como uma rede pode ser formada com esses elementos processadores;

1949: Donald Hebb [Hebb, 1949] propõe um postulado que determina como ocorre a modificação das ligações sinápticas entre neurônios;

1958: Frank Rosenblatt [Rosenblatt, 1958] propõe o modelo Perceptron de rede neural, sendo este capaz de classificar padrões;

1960: Widrow e Hoff [Widrow \& M.E., 1960] propõem a Regra Delta de aprendizado;

1969: Minsky e Papert [Minsky \& A., 1969] expõem as limitações do modelo Perceptron, desestimulando o avanço das pesquisas na área, nos anos que se seguem;

1982: John Hopfield [Hopfield, 1982] faz ressurgir o interesse no estudo de redes neurais realimentadas, propondo um modelo com propriedades de memória associativa, semelhante ao anteriormente desenvolvido por Little [Little, 1974]. Hopfield trouxe o rigor matemático da Mecânica Estatístca ao campo de RNAs, fazendo analogias destas a sistemas físicos;

1986: Rumelhart e McClelland [Rumelhart \& McClelland, 1986] redescobrem o algoritmo de aprendizado Back-Propagation, inicialmente introduzido por Werbos [Werbos, 1974]. Eles mostram como treinar uma rede estática de múltiplas camadas, o que culmina em uma explosão de pesquisas na área, nas décadas seguintes.

\subsection{Conclusão}

As RNAs constituem um paradigma de computação diferente do paradigma convencional, que se baseia em um elemento processador central controlando todo o sistema. No paradigma neural, o processamento é realizado de forma distribuída, por meio de neurônios artificiais. Diferentemente de um fluxo seqüencial de instruções que um computador digital tem que seguir, nas RNAs têm-se elementos processadores que operam de maneira paralela, interagindo uns com os outros. Não existe um programa explícito, sendo que o funcionamento do modelo depende da dinâmica dos neurônios, e da forma como esses são conectados, o que determina o tipo de tarefa que a rede realizará. Normalmente, ao invés de ser programada, uma rede ou "máquina neural" aprende a resolver uma tarefa que lhe é atribuída por meio de um algoritmo de treinamento ou aprendizado, que ajusta os parâmetros da mesma.

Os modelos de RNAs podem ser dividos em duas classes: os dinâmicos e os estáticos, dependendo da existência ou não de realimentações entre os neurônios, respectivamente. No 
próximo capítulo, é apresentado o modelo de Hopfield, que constitui um modelo de rede neural dinâmica ou realimentada, capaz de operar como uma memória associativa. 


\section{Capítulo 4}

\section{O modelo de Hopfield}

Até a publicação do artigo "Redes neurais e sistemas físicos com habilidades computacionais coletivas emergentes" [Hopfield, 1982], por J. J. Hopfield, em 1982, havia pouco interesse no estudo de redes neurais realimentadas, dada a falta de conhecimento de como treiná-las, e a dificuldade em analisá-las. A principal contribuição de Hopfield foi estabelecer uma analogia entre o seu modelo de rede neural e o sistema de spins de Ising [Ising, 1924]. Tal analogia comparava os neurônios da rede, que possuem estado de ativação binário, com átomos de um material ferromagnético, que também apresentam estados binários, dados pela orientação dos spins (spin up e spin down). Hopfield foi então capaz de aplicar resultados formais da Mecânica Estatística para a análise de seu modelo, encontrando uma função de energia que garante a estabilidade da rede.

Hopfield investigou também as propriedades computacionais do modelo por ele formulado, utilizando, para isso, uma regra de aprendizado para fixar as sinapses da rede. Dessa forma, ele demonstrou que a rede neural era capaz de "escolher" entre configurações de estados preferidas, exibindo, em certo sentido, uma espécie de memória. Considerando que essas configurações de estado representavam informações que podiam ser armazenadas, Hopfield mostrou que a rede era capaz de recuperar tais informações com base no conteúdo das mesmas, como em uma memória associativa. Além disso, certas características da memória exibida pelo modelo, fez com que hipóteses fossem levantadas acerca de semelhanças com o cérebro:

- Informações são armazenadas de forma distribuída na rede, não sendo associadas a endereços físicos específicos, como acontece na memória dos computadores digitais;

- Informações podem ser recuperadas como resultado da evolução do sistema em direção a um atrator, e não por uma busca exaustiva, como ocorre nos computadores digitais;

- Informações são acessadas com base no conteúdo das mesmas, podendo-se utilizar fragmentos de informação muitas vezes inconsistentes. 


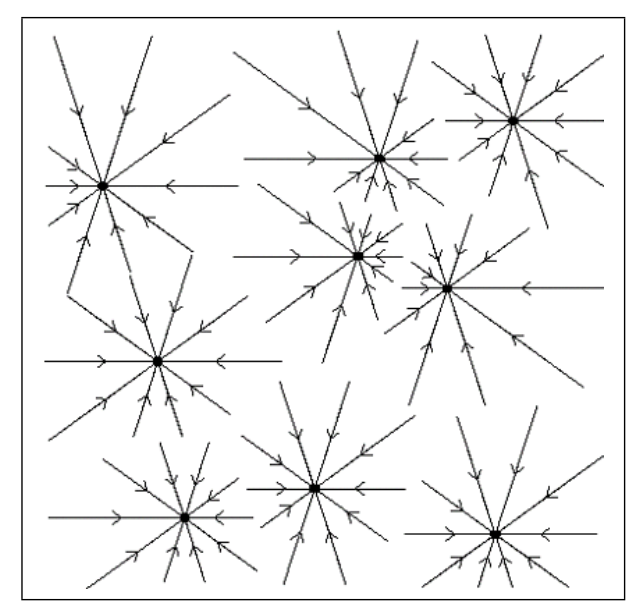

Figura 4.1: Memórias armazenadas como pontos de equilíbrio.

De acordo com o o modelo de Hopfield, a busca por um item de memória se inicia com a especificação do estado ou condição inicial da rede, que representa a porção conhecida da informação buscada. Conforme o estado do sistema evolui no tempo, ele converge para um dado ponto de equilíbrio, que representa o item de memória mais próximo ao procurado. Um certo nível de ruído é tolerado na informação utilizada como chave de busca, a qual deve estar contida na bacia de atração daquele atrator referente ao item de memória procurado. A Figura 4.1 dá uma ilustração do princípio de memória exibido pelo modelo de Hopfield, onde os pontos representam atratores que codificam os itens de memória armazenados, enquanto as setas representam condições iniciais, usadas como chaves de busca na recuperação de informações.

\subsection{Equações da dinâmica}

O modelo de Hopfield é uma rede neural formada por neurônios de McCulloch e Pitts (Seção 3.2), sendo descrita pelas seguintes equações de diferenças (modelo discreto):

$$
\left\{\begin{array}{l}
v_{i}(k+1)=\operatorname{sgn}\left(u_{i}(k)\right), \\
u_{i}(k)=\sum_{j=1}^{n} T_{i j} v_{j}(k)+I_{i}, \quad i=1, \ldots, n .
\end{array}\right.
$$

onde $n$ é o número de neurônios da rede, $u_{i}: \mathbb{Z} \rightarrow \mathbb{R}, i=1, \ldots, n$ é o chamado estado interno do $i$-ésimo neurônio, $I_{i}$ é uma constante ou bias, $v_{i}: \mathbb{Z} \rightarrow B(B=\{-1,1\})$ é a saída ou resposta emitida pelo neurônio (disparando, para $v_{i}(k)=1$, ou em repouso, para $v_{i}(k)=-1$ ) e $k \in \mathbb{Z}$ representa o tempo. O parâmetro $T=\left[T_{i j}\right] \in \mathbb{R}^{n \times n}$ codifica a eficiência sináptica entre dois neurônios $i$ e $j$, e a função $s g n: \mathbb{R} \rightarrow B$ é a função de ativação (função sinal), dada por 


$$
\operatorname{sgn}\left(u_{i}\right)=\left\{\begin{array}{lll}
1, & \text { se } & u_{i} \geq 0 \\
-1, & \text { se } & u_{i}<0
\end{array}\right.
$$

e cujo gráfico é dado pela Figura 3.2(b), no Capítulo 3.

O modelo analógico, ou contínuo, de Hopfield, é dado pelo seguinte conjunto de equações diferenciais:

$$
\left\{\begin{array}{l}
\dot{u}_{i}=-b_{i} u_{i}(t)+\sum_{j=1}^{n} T_{i j} v_{j}(t)+I_{i}, \\
v_{j}(t)=h\left(u_{i}(t)\right), \quad i=1, \ldots, n .
\end{array}\right.
$$

onde $u_{i}: \mathbb{R} \rightarrow \mathbb{R}, i=1, \ldots, n$ é diferenciável, $v_{i}: \mathbb{R} \rightarrow B(B=\{-1,1\}), t \in \mathbb{R}$, e os demais parâmetros são como no modelo discreto (equação (4.1)). A função $h$ é a função sigmoidal (ver Figura 3.2(a)). A seguir, a estabilidade da rede é analisada.

\subsection{Análise de estabilidade}

\subsubsection{Modelo contínuo}

A estabilidade do modelo de Hopfield é aqui provada por um argumento semelhante ao que se utiliza com funções de Lyapunov (ver Teorema 2.5.9, na Seção 2.5.4). Para isso, determina-se uma função de energia do sistema, que não necessariamente é uma função de Lyapunov (por não se garantir que essa é sempre definida positiva (ver Definição 2.5.8)), mas que, mesmo assim, permite tirar conclusões acerca da estabilidade do sistema. Utilizando o Método do Gradiente Variável (ver Seção 2.5.4), seja $E: \mathbb{R}^{n} \rightarrow \mathbb{R}$ essa função de energia procurada. A derivada $\dot{E}(v)$, ao longo de soluções de (4.3), é dada por:

$$
\dot{E}(v)=\sum_{j=1}^{n} \frac{\partial E}{\partial v_{i}} \frac{\partial v_{i}}{\partial u_{i}} \frac{\partial u_{i}}{\partial t}
$$

pela regra da cadeia. Suponha $g=\frac{\partial E}{\partial v_{i}}$, tal que $g: \mathbb{R}^{n} \rightarrow \mathbb{R}^{n}$ é $C^{2}$ (duas vezes diferenciável). Logo, para $g(v)=\left(g_{1}(v), \ldots, g_{n}(v)\right)$, tem-se:

$$
\dot{E}(v)=\sum_{j=1}^{n} g_{i} \frac{\partial v_{i}}{\partial u_{i}} \frac{\partial u_{i}}{\partial t}
$$

Como se procura uma função $E(v)$ tal que $\dot{E}(v)<0$ (definida negativa), é possível escolher $g=-\frac{d u}{d t}$, o que satisfaz essa condição $\left(\frac{\partial v_{i}}{\partial u_{i}}>0\right.$, pois a função $h$ em (4.3) é monotônica crescente). No entanto, a existência da função $g(v)$ está condicionada à restrição 


$$
\frac{\partial g_{i}}{v_{j}}=\frac{\partial g_{j}}{v_{i}}, \quad \forall i, j=1, \ldots, n .
$$

de acordo com a Proposição 2.5.11. Pode-se verificar que esta condição é satisfeita se as sinapses da rede (4.3) forem escolhidas como

$$
T_{i j}=T_{j i}, \quad i, j=1, \ldots, n
$$

o que estabelece uma condição de simetria nas conexões entre os neurônios. Dada a escolha $g=-\frac{d u}{d t}$, tem-se:

$$
\dot{E}(v)=-\sum_{j=1}^{n} \frac{\partial v_{i}}{\partial u_{i}}\left(\frac{\partial u_{i}}{\partial t}\right)^{2} \leq 0,
$$

como desejado. A função $E$ procurada pode então ser obtida integrando (ver equação (2.30)):

$$
\begin{aligned}
E & =\int_{0}^{v} g(\eta)^{T} \cdot d \eta=\int_{0}^{v_{i}} \sum_{i=1}^{n} g_{i}(\eta) d \eta_{i} \\
& =-\frac{1}{2} \sum_{i=1}^{n} \sum_{j=1}^{n} T_{i j} v_{i} v_{j}+\sum_{i=1}^{n} I_{i} v_{i}+\sum_{i=1}^{n} \int_{0}^{v_{i}} h^{-1}(\eta) d \eta
\end{aligned}
$$

Como a função $E(v)$ acima não é necessariamente definida positiva, não é possível aplicar o Teorema de Lyapunov (Teorema 2.5.9). No entanto, cabe observar que:

- $E(v)$ é limitada inferiormente, pois $\left|v_{i}\right| \leq 1$ e o termo $\int_{0}^{v_{i}} h^{-1}(\eta) d \eta$ em (4.5) é estritamente positivo ${ }^{1}$, como mostra a Figura 4.2, para a função $h$ cujo gráfico é dado na Figura 4.3 ;

- A derivada $\dot{E}(v)$ sempre decresce, anulando-se apenas quando $\dot{u}=0$, o que ocorre somente nos pontos de equilíbrio do sistema.

Logo, $E(v)$ não admite oscilações e, conseqüentemente, uma solução de (4.3) sempre convergirá para um ponto de equilíbrio, não apresentando dinâmica periódica ou caótica. Os pontos de equilíbrios são, portanto, assintoticamente estáveis, correspondendo a mínimos (locais) de $E(v)$, como ilustra a Figura 4.4 .

\footnotetext{
${ }^{1}$ Uma forma específica para a função sigmoidal $h$ teve de ser escolhida. Essa forma utilizada foi $h\left(u_{i}\right)=\left(\frac{2}{\pi}\right) \tan ^{-1}\left(\lambda \pi u_{i}\right)$, onde $\lambda>0$.
} 


\subsubsection{Modelo discreto}

Para a prova da estabilidade do modelo discreto (equação (4.1)), utiliza-se a seguinte função de energia:

$$
E(x)=-\frac{1}{2} \sum_{i} \sum_{j, j \neq i} w_{i j} x_{i}(n) x_{j}(n)
$$

Para encontrar a variação discreta de $E(v)$, ao longo de soluções de (4.1) (analogamente ao que foi feito para (4.3), na seção anterior), deve-se considerar, nesse caso, as seguintes hipóteses:

(H1) A atualização do estado da rede é assíncrona, isto é, somente um neurônio troca de estado a cada instante de tempo;

(H2) Os neurônios não são conectados a si próprios $\left(T_{i i}=0\right)$;

(H3) As conexões são simétricas (isto é, $T_{i j}=T_{j i}$ );

Tem-se que a variação da função de energia, ao longo de soluções de (4.1), devida à mudança de estado de apenas um neurônio da rede (por (H1)), é:

$$
\begin{aligned}
\Delta E(v) & =-\frac{1}{2} \sum_{i} \sum_{j, j \neq i} T_{i j} v_{i}(n+1) v_{j}(n)+\frac{1}{2} \sum_{i} \sum_{j, j \neq i} T_{i j} v_{i}(n) v_{j}(n) \\
& =\left(-v_{i}(n+1)+v_{i}(n)\right) \sum_{j, j \neq i} T_{i j} v_{j}(n) \\
& =\left(-v_{i}(n+1)+v_{i}(n)\right) v_{i}(n+1) \\
& =-\left(v_{i}(n+1)\right)^{2}+v_{i}(n) v_{i}(n+1) \\
& \leq 0
\end{aligned}
$$

A passagem da primeira para a segunda linha da equação anterior decorre das hipóteses (H2) e (H3). Da segunda para a terceira linha, foi utilizada a equação (4.1). Como conseqüência desse

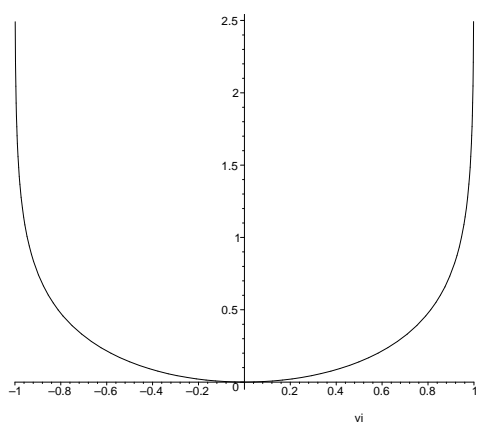

Figura 4.2: Gráfico de $\int_{0}^{v_{i}} h^{-1}(\eta) d \eta$.

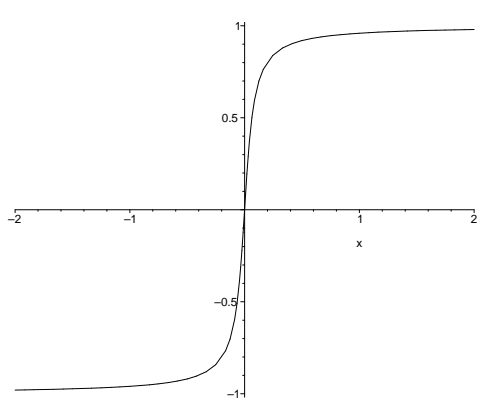

Figura 4.3: Gráfico de $h\left(u_{i}\right)$. 


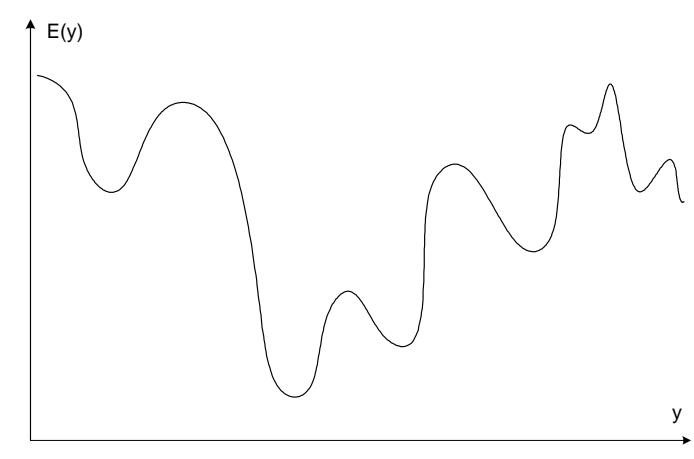

Figura 4.4: Representação da função de energia $E(v)$.

resultado, vale a mesma argumentação à respeito da estabilidade para o caso contínuo: $E(v)$ é limitada; $\Delta E(v)$ sempre decresce; $\Delta E(v)=0$ somente nos pontos fixos. Daí, concluí-se que $v(k)$ não pode oscilar, e só existem pontos fixos assintoticamente estáveis para (4.1).

\subsection{Armazenamento de padrões}

Hopfield se valeu do seguinte postulado de Hebb [Hebb, 1949] para derivar uma regra de aprendizado da rede:

“Quando dois neurônios são simultaneamente ativados, a ligação sináptica entre eles é fortalecida".

Este postulado pode ser representado pela fórmula:

$$
\Delta T_{i j}=\alpha v_{i} v_{j}
$$

para um dado padrão ${ }^{2}$ de memória $v=\left[v_{1}, \ldots v_{n}\right]^{T} \in B(B=\{-1,1\})$ e para a taxa de aprendizado $\alpha>0$. Como os componentes de $v$ são $v_{i}= \pm 1$ (que representam o estado de ativação dos neurônios), sempre que $v_{i}=v_{j}$ (o que representa que os neurônios $i$ e $j$ são simultaneamente ativados), a variação $\Delta T_{i j}$ é positiva, correspondendo ao fortalecimento da sinapse entre tais neurônios. Além disso, a regra dada por (4.8) enfraquece as sinapses quando $v_{i} \neq v_{j}$, pois dessa forma a variação $\Delta T_{i j}$ é negativa.

Para o armazenamento de $m$ padrões $v^{p} \in B, p=1, \ldots, m$, a regra dada por (4.8) resulta na soma:

$$
T=\sum_{p=1}^{m} v^{p}\left(v^{p}\right)^{T}
$$

\footnotetext{
${ }^{2}$ Um padrão é considerado aqui um vetor binário que representa um item de memória.
} 
Essa soma é chamada de outer products, pois calcula as "correlações" entre os termos $v^{p}$, $p=1, \ldots, m$. Além disso, a regra de aprendizado utilizada considera

$$
I=0
$$

em (4.3) e (4.1). Na próxima seção, demonstra-se que os parâmetros assim escolhidos, garantem o armazenamento e a recuperação dos padrões $v^{p}$.

Observação 4.3.1 Pelo regra dada por (4.9), percebe-se que o aprendizado da rede não involve um processo iterativo, como ocorre no treinamento de outros modelos de redes neurais da literatura. Os valores das sinapses são calculados em um único passo, usando-se apenas os padrões de treinamento, que são considerados como protótipos de treinamento. 


\subsection{Recuperação de padrões}

Considere o modelo discreto de Hopfield, dado pela equação (4.1). A recuperação de um item de memória consiste em se fixar a condição inicial do sistema com a informação (possivelmente parcial) do item de memória que é procurado. A regra de aprendizado dada por (4.9) e (4.10) garante que os padrões $v^{p}, p=1, \ldots, m$ são armazenados como pontos fixos assintoticamente estáveis de (4.1). Para verificar isso, considere que o sistema se encontra no estado $v(k)=v^{p}$. De (4.1), tem-se:

$$
v(k+1)=\operatorname{sgn}\left(T v^{p}\right)=\operatorname{sgn}\left(\left[\sum_{j=1}^{m} v^{j}\left(v^{j}\right)^{T}\right] v^{p}\right)=\operatorname{sgn}\left(n v^{p}\right)=v^{p}
$$

isto é, cada padrão $v^{p}, p=1, \ldots, m$ é um equilíbrio de (4.1). Além disso, se os padrões $v^{p}$ forem mutuamente ortogonais (isto é, $\left(v^{j}\right)^{T} v^{p}=0$ para $j \neq p$ ), então, de (4.6), segue que

$$
E\left(v^{p}\right)=-n^{2} / 2, \quad p=1, \ldots, m
$$

já que todos os componentes de $v^{p}$ são $v_{i}^{p} \pm 1, i=1, \ldots, n$. Portanto, $E(v)$ assume mínimo em cada $v^{p}$, sendo estes estados assintoticamente estáveis de (4.1). Caso os padrões não sejam mutuamente ortogonais, $E(v)$ não necessariamente assume mínimo em $v^{p}$. Nesse caso, os padrões $v^{p}$ não são necessariamente pontos fixos de (4.1), pois:

$$
v(k+1)=\operatorname{sgn}\left(T v^{p}\right)=\operatorname{sgn}\left(\left[\sum_{j=1}^{m} v^{j}\left(v^{j}\right)^{T}\right] v^{p}\right)=\operatorname{sgn}\left(n v^{p}+\left[\sum_{j=1, j \neq k}^{m} v^{j}\left(v^{j}\right)^{T} v^{p}\right]\right),
$$

onde

$$
\sum_{j=1, j \neq k}^{m} v^{j}\left(v^{j}\right)^{T} v^{p}
$$

representa um termo de "ruído", que se tiver algum componente com magnitude maior que $n$, e sinal oposto que o correspondente componente de $v^{p}$, não permite que $v^{p}$ seja um equilíbrio do sistema. Nesse caso, é criado outro ponto de equilíbrio no sistema, que é dito um estado espúrio, pois não corresponde a nenhum dos itens de memória armazenados. Assim, tem-se que o termo de ruído (4.11) aumenta em magnitude conforme o número de padrões $m$ a serem armazenados também aumenta, o que ocasiona em uma maior chance de ocorrência de estados espúrios na rede. 


\subsection{Simulação}

A seguir, é apresentada uma simulação do modelo discreto de Hopfield (equação (4.1)), funcionando como uma memória associativa que armazena imagens binárias. O sistema apresenta $n=841$ neurônios, que são associados aos pixels de imagens com dimensão de 29x29 pixels. Os $m=10$ padrões $\operatorname{armazenados}^{3}$ são apresentados na Figura 4.5.

Utilizando-se como condição inicial do sistema uma versão corrompida com ruído do $1^{\circ}$ padrão da Figura 4.5, a evolução do estado da rede produz à recuperação do padrão original (Figura 4.6). Observa-se que com poucas iterações (apenas quatro), já se tem a recuperação completa do padrão armazenado.

Para ilustrar a existência de estados espúrios, mais ruído foi adicionado ao padrão anteriormente recuperado. Usando-o como condição inicial do sistema, nota-se (Figura 4.7) que após algumas iterações, ocorre a convergência para um ponto fixo que não corresponde a nenhum dos itens armazenados, sendo, portanto, um estado espúrio do sistema.

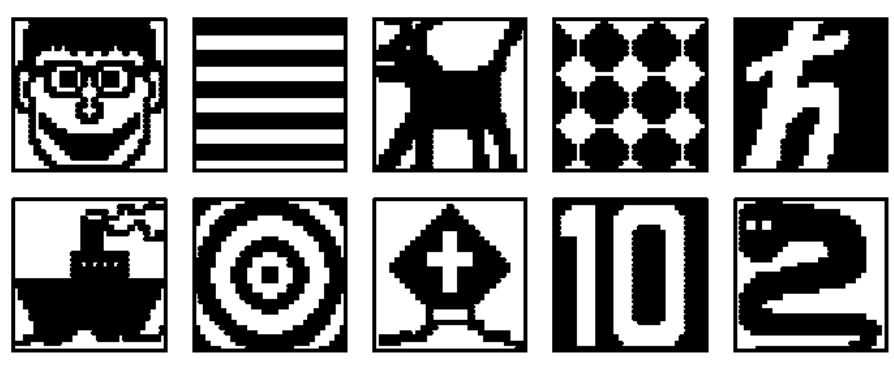

Figura 4.5: Exemplo de padrões armazenados.

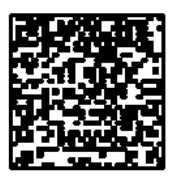

$n=0$

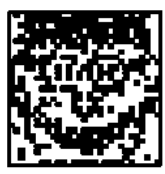

$n=1$

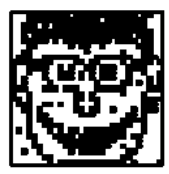

$n=2$

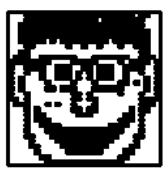

$n=3$

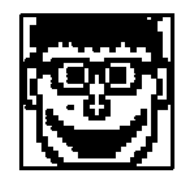

$n=4$

Figura 4.6: Exemplo de recuperação de padrão com ruído.

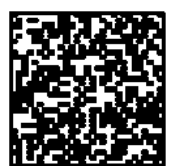

$n=0$

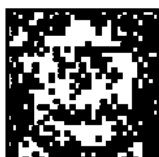

$n=1$

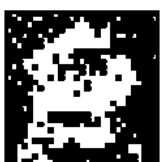

$n=2$

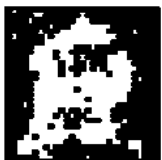

$n=3$

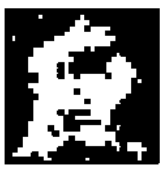

$n=4$

Figura 4.7: Exemplo de convergência para estado espúrio.

\footnotetext{
${ }^{3}$ Imagens obtidas de [Coolen, 1998].
} 


\subsection{Discussão}

O modelo de Hopfield é também utilizado na resolução de problemas de otimização [Hopfield \& Tank, 1985]. Como uma memória associativa, este modelo computacional define um conceito de memória diferente do convencional. Acredita-se que este princípio de funcionamento de memória na rede seja semelhante ao exibido no cérebro, pois tem-se o armazenamento distribuído da informação, o processamento paralelo e a recuperação de padrões pela evolução do estado do sistema no tempo, com base no conteúdo da informação procurada.

Foi também apresentado, neste capítulo, a análise de estabilidade da rede, tanto para o modelo contínuo, quanto para o modelo discreto. Como parte da análise do modelo, cabe ressaltar suas limitações:

- Existe a formação de estados indesejáveis (estados espúrios) na rede;

- A capacidade de armazenamento é limitada, sendo estimada experimentalmente em $p=0.15 n$ padrões (onde $n$ representa a quantidade de neurônios da rede). Esse valor pode ser melhorado utilizando-se outras estratégias de armazenamento de padrões [Personnaz et al., 1986].

Além disso, certas características do modelo de Hopfield o tornam pouco biologicamente plausível, como um modelo do cérebro:

- Os neurônios são acoplados globalmente, isto é, existem sinapses conectando cada neurônio a todos outros da rede;

- O neurônios são conectados de forma simétrica;

- Pontos de equilíbrio não são comuns na dinâmica do cérebro, que apresenta comportamento oscilatório e, em certas condições, até caótico [Skarda \& Freeman, 1982].

Apesar das limitações apresentadas, o modelo de Hopfield se mostra representativo como um modelo de processamento de informação que se utiliza de propriedades de sistemas dinâmicos. No próximo capítulo, é apresentado um modelo de rede neural que tenta suplantar algumas dessas limitações discutidas. Esse modelo compreende as chamadas Redes Neurais Celulares. 


\section{Capítulo 5}

\section{Redes Neurais Celulares}

Para efetivamente se explorar o paralelismo de uma RNA, sua implementação deve ser feita em hardware. No entanto, isso normalmente é inviável, devido ao alto número de conexões que podem existir entre os elementos processadores. No modelo de Hopfield, por exemplo, os neurônios são totalmente conectados uns aos outros, produzindo, para uma rede com $n$ neurônios, um total de $n^{2}$ conexões. Como o crescimento do número de conexões, em função do número de neurônios, é de ordem quadrática $\left(O\left(n^{2}\right)\right)$, a construção de uma rede de Hopfield mesmo em hardware VLSI ${ }^{1}$ é inviável, para $n$ grande. As alternativas propostas para sanar tal problema consistem basicamente de: técnicas de multiplexação de sinais (hardwares ópticos, por exemplo); diferentes arquiteturas/topologias de interconexões, ambas visando a diminuição do número de conexões entre os elementos processadores. Nesse capítulo, é apresentado um modelo de rede neural que explora essa segunda alternativa de solução ao problema discutido.

Em 1988, L. Chua propôs o modelo de Redes Neurais Celulares (RNCs) [Chua \& Yang, 1988], que tratam-se de redes localmente acopladas, isto é, redes cujas interconexões entre os neurônios se restringem a uma certa vizinhança dos mesmos. Esse tipo de rede é ilustrado na Figura 5.1, onde células (ou neurônios) são representadas por círculos preenchidos, enquanto as conexões entre elas são representadas por arestas ligando tais círculos.

As Redes Neurais Celulares são assim denominadas por apresentarem certa semelhança com os Autômatos Celulares ${ }^{2}$, em relação à conectividade local presente entre as células da rede. Essa característica, em conjunto com uma implementação em hardware analógico da rede, fazem com que as RNCs sejam especialmente eficientes na execução de aplicações que envolvam grandes quantidades de cálculos realizados apenas à nível local, ou seja, envolvendo apenas dados de vizinhanças de pontos ou células de um reticulado (grid). Esse é o caso, por exemplo, de aplicações em Processamento de Imagens ou de Solução Numérica (discretização)

\footnotetext{
${ }^{1}$ Very Large Scale Integration ou Integração [de componentes eletrônicos] em Alta Escala.

${ }^{2}$ Sistemas dinâmicos discretos no tempo e espaço, localmente acoplados, cujos estados também são discretos.
} 
de equações Diferenciais Parciais (EDPs). Entretanto, as aplicações das RNCs não se limitam apenas a esse tipo de tarefa, como será visto no Capítulo 6.

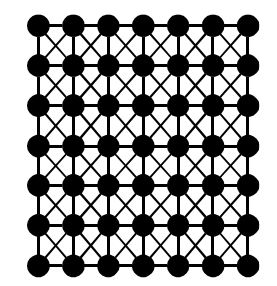

Figura 5.1: Representação espacial de uma RNC.

\subsection{Equações da dinâmica}

A dinâmica do sistema é dada pelo seguinte sistema de equações diferenciais:

$$
\left\{\begin{aligned}
\frac{d x_{i j}(t)}{d t} & =-x_{i j}(t)+\sum_{(k, l) \in N_{r}(i, j)} A_{i j, k l} y_{k l}(t)+\sum_{(k, l) \in N_{r}(i, j)} B_{i j, k l} u_{k l}+I \\
y_{k l} & =\operatorname{sat}\left(x_{k l}\right)
\end{aligned}\right.
$$

para $1 \leq i \leq M$ e $1 \leq j \leq N$, onde $x_{i j}: \mathbb{R} \rightarrow \mathbb{R}$ é uma função diferenciável que representa o estado de uma célula, $u_{i j} \in \mathbb{R}$ é uma constante que representa um estímulo ou entrada externa e $y_{i j}: \mathbb{R} \rightarrow D(D=\{x \in \mathbb{R}:-1 \leq x \leq 1\})$ representa a saída. O parâmetro $I \in \mathbb{R}$ é uma constante e a variável independente $t \in \mathbb{R}$ representa o tempo. As matrizes $A \in \mathbb{R}^{M N \times M N} \mathrm{e}$ $B \in \mathbb{R}^{M N \times M N}$ determinam as conexões das células, que são consideradas como se estivessem dispostas em um reticulado $M \mathrm{x} N$ dimensional, como ilusta a Figura 5.1. A função de ativação sat $: \mathbb{R} \rightarrow \mathbb{R}$ é a função saturação, dada por:

$$
\operatorname{sat}\left(x_{i j}\right)= \begin{cases}-1, & \text { se } x_{i j}<-1 \\ x_{i j}, & \text { se }-1 \leq x_{i j} \leq 1 \\ 1, & \text { se } x_{i j}>1\end{cases}
$$

e cujo gráfico é dado na Figura 3.2(d) (ver Seção 3.2). A vizinhança de raio $r$, em torno de uma célula, é denotada por $N_{r}(i, j)$, e definida como:

$$
N_{r}(i, j)=\{(k, l) \mid \max (|k-i|,|l-j|) \leq r\}, \quad 1 \leq k \leq M, \quad 1 \leq l \leq N .
$$

Essa definição pode ser melhor visualizada com a ajuda da Figura 5.2. Pela definição, cada célula tem $(2 \cdot r+1)^{2}$ vizinhas, incluindo a própria célula considerada. 


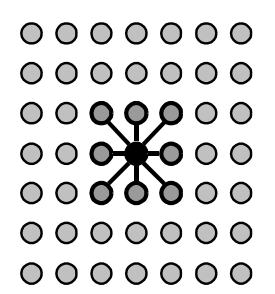

(a) $r=1$

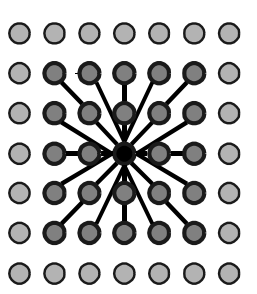

(b) $r=2$

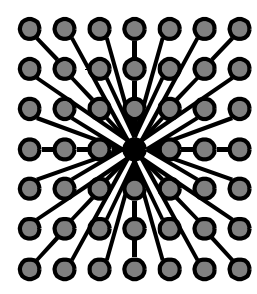

(c) $r=3$

Figura 5.2: Exemplos de vizinhanças de uma célula.

No modelo considerado, as seguintes restrições são impostas:

$$
\begin{aligned}
& \left|x_{i, j}(0)\right| \leq 1 \\
& \left|u_{i, j}\right| \leq 1 \\
& A_{i, j ; k, l}=A_{k, l ; i, j}
\end{aligned}
$$

para $1 \leq i \leq M$ e $1 \leq j \leq N$

\subsection{Análise de estabilidade}

Considere a seguinte equação vetorial, associada à (5.1), que descreve a dinâmica da rede:

$$
\left\{\begin{array}{l}
\dot{x}=-x+T y+I, \\
y=\operatorname{sat}(x)
\end{array}\right.
$$

onde $T=\left[T_{i j}\right] \in \mathbb{R}^{n \times n}, n=M N, T=A, x: \mathbb{R} \rightarrow \mathbb{R}^{n}$ é diferenciável, $y: \mathbb{R} \rightarrow D^{n}\left(D^{n}=\right.$ $\left.\left\{x \in \mathbb{R}^{n}:-1 \leq x_{i} \leq 1, i=1, \ldots, n\right\}\right), \operatorname{sat}(x)=\left[\operatorname{sat}\left(x_{1}\right), \ldots, \operatorname{sat}\left(x_{n}\right)\right]^{T}$ e $I \in \mathbb{R}^{n}$, sendo $M, N, A, B$ como definidos na equação (5.1). Note que as seguintes modificações que foram introduzidas em relação à (5.1): o parâmetro $I$ foi generalizado para um vetor $n$-dimensional, pois essa forma será útil nas aplicações envolvendo memória associativa; a matriz $A$ foi trocada por $T$ e a matriz $B$ foi igualada a zero $(B=0)$, para tornar a notação semelhante à empregada no modelo de Hopfield (Capítulo 4). Estas modificações não interferem nos resultados obtidos quanto a estabilidade de (5.1), sendo apenas introduzidas para simplificação da análise.

Suponha $T$ simétrica em (5.4), e considere a seguinte função de energia $E: D^{n} \rightarrow \mathbb{R}$ $\left(D^{n} \subset \mathbb{R}^{n}\right)$ :

$$
E(y)=-\frac{1}{2} y^{T} T y+\frac{1}{2} y^{T} y-y^{T} I
$$

É possível mostrar (ver Teorema B.3.6 no Apêndice B) que:

(a) A função de energia (5.5) decresce monoticamente ao longo de uma solução de (5.4); 
(b) Toda solução de (5.4) converge para um ponto de equilíbrio.

Como a função sat $(x)$ em (5.4) não é diferenciável, existe uma dificuldade técnica para provar tais afirmações. Dispõe-se de duas alternativas:

(a) Definir soluções ditas "locais" para (5.1), como realizado em [Li et al., 1989];

(b) Supor a derivada de $f$ igual a zero quando $\left|x_{i}\right|=1$, conforme apresentado em [Chua \& Yang, 1988].

O Apêndice B apresenta a prova do Teorema B.3.6 pela primeira opção, por esta ser matematicamente mais "elegante".

\subsection{Programação de uma RNC}

Pode-se dizer que uma RNC é programada quando os valores de seus parâmetros são ajustados, a fim de obter um comportamento desejado. Esses parâmetros podem ser determinados por meio de:

- "heurísticas", derivadas de conhecimento prévio de determinado domínio de aplicação;

- algoritmos de aprendizado, como ocorre em outros modelos de redes neurais (ver Seção $3.3)$.

Na próxima seção, será ilustrada a escolha dos parâmetros de RNCs para resolver problemas em Processamento de Imagens, por meio de conhecimento prévio neste domínio. No próximo capítulo, algoritmos de aprendizado serão utilizados para se implementar uma memória associativa em uma RNC.

\subsection{RNCs para o processamento de imagens}

Estudos fisiológicos da retina confirmam que as células nervosas dessa região apresentam um padrão de interconexão local, o que é compartilhado pela estrutura das RNCs [Werblin et al., 1995]. Assim, tais redes podem ser utilizadas para modelarem certas funções do processamento de baixo-nível de visão, como:

- Detecção de movimento;

- Extração de contornos;

- Suavização de imagens; 
- Detecção de estímulos direcionais (linhas verticais, horizontais, etc.).

A seguir, será visto como uma RNC pode ser configurada para simular algumas destas funções citadas. Por serem realizáveis em hardware, as RNCs são atrativas para compor um sistema de visão computacional, devido ao desempenho em tempo real requerido por aplicações desse tipo.

\subsubsection{Máscaras ou moldes de clonagem}

Em problemas de processamento de imagens, aplica-se em geral uma mesma operação a todos os pixels (ou células) de uma imagem. Em uma RNC a ser utilizada para processar imagens, isso equivale a dizer que na equação (5.1) as linhas da matriz $A$ serão iguais, o mesmo ocorrendo com as linhas da matriz $B$. Dessa forma, uma representação conveniente para cada linha de $A$ e $B$ é dada pela "máscara" ou "molde de clonagem" (considerando-se uma vizinhança de raio 1$)$ :

$$
\bar{A}=\left(\begin{array}{ccc}
\bar{A}_{-1,-1} & \bar{A}_{-1,0} & \bar{A}_{-1,1} \\
\bar{A}_{0,-1} & \bar{A}_{0,0} & \bar{A}_{0,1} \\
\bar{A}_{1,-1} & \bar{A}_{1,0} & \bar{A}_{1,1}
\end{array}\right) \quad e \quad \bar{B}=\left(\begin{array}{lll}
\bar{B}_{-1,-1} & \bar{B}_{-1,0} & \bar{B}_{-1,1} \\
\bar{B}_{0,-1} & \bar{B}_{0,0} & \bar{B}_{0,1} \\
\bar{B}_{1,-1} & \bar{B}_{1,0} & \bar{B}_{1,1}
\end{array}\right)
$$

onde

$$
\bar{A}_{k-i, l-j}=A_{i j, k l}
$$

$\mathrm{e}$

$$
\bar{B}_{k-i, l-j}=B_{i j, k l}
$$

para $k, l \in N_{r}(i, j)$. Nesse caso, as máscaras ilustram uma vizinhança de raio $r=1$, mas a definição se estende também para vizinhanças de raio maiores. As posições centrais, tanto em $\bar{A}$ quanto em $\bar{B}$ (elementos $\bar{A}_{0,0}$ e $\bar{B}_{0,0}$, respectivamente), denotam a conexão de cada célula consigo própria. Essas matrizes são denominadas moldes de clonagem pois o mesmo padrão de conexão entre células é "clonado", isto é, utilizado por todas as células que compõem a rede.

Nos exemplos seguintes, a especificação dos parâmetros $A$ e $B$ em (5.1) são feitos por meio de moldes de clonagem. Com esta representação, torna-se mais fácil visualizar o relacionamento que cada célula tem com a sua vizinhança e, conseqüentemente, entender o tipo de processamento que está ocorrendo na rede. 


\subsubsection{Suavização (remoção de ruídos) em imagens}

A suavização de uma imagem tende a deixá-la visualmente mais agradável por meio da diminuição de variações bruscas de intensidade na mesma, o que pode corresponder a ruídos mas, também, a contornos desta. Um algoritmo de suavização deve remover os possíveis ruídos, mas preservar os contornos da imagem. Diversos são os métodos para se conseguir esse efeito [Rosenfeld \& Kak, 1982], sendo que aqui será demonstrado a utilização do operador Laplaciano [Lim, 1990] para tal fim. O Laplaciano de uma função $\psi(x, y)$, de classe $C^{2}$ definida no plano, é dado por

$$
\Delta^{2} \psi=\frac{\partial^{2} \psi}{\partial x^{2}}+\frac{\partial^{2} \psi}{\partial y^{2}}
$$

que consiste da soma das derivadas parciais segundas de $\psi$. Discretizando esse operador, por meio de aproximações de diferenças centrais baseadas em uma expansão em série de Taylor [Press et al., 1993], obtém-se

$$
\Delta^{2} \psi \approx \frac{1}{\delta h^{2}}[\psi(x+\delta h, y)+\psi(x, y+\delta h)+\psi(x-\delta h, y)+\psi(x, y-\delta h)-4 \psi(x, y)]
$$

onde $\delta h$ é passo de discretização. Suponha que a função $\psi$ considerada represente a imagem a ser processada pela RNC. Essa discretização pode então ser implementada pelo seguinte molde de clonagem:

$$
\bar{A}=\left(\begin{array}{rrr}
0.0 & 1.0 & 0.0 \\
1.0 & -4.0 & 1.0 \\
0.0 & 1.0 & 0.0
\end{array}\right)
$$

É possível mostrar que uma RNC configurada com esse molde de clonagem suaviza o sinal ou imagem atribuído à condição inicial $x(0)$ do sistema [Crounse \& Chua, 1997]. A resposta em frequiência da RNC, quando configurada com esse operador $\bar{A}$ (que denota a resposta em impulso do sistema), tende a acentuar os componentes de baixa freqüência e atenuar os de alta freqüência do sinal em $x(t)$, "suavizando-o". Os outros parâmetros da rede são fixados em zero ( $B=0$ e $I=0$ em (5.1)), nesse exemplo de aplicação. Na próxima seção, será mostrado que esse mesmo operador Laplaciano, quando configurado como o parâmetro $B$ de (5.1), pode ser usado também para extrair contornos em imagens.

Outra forma de suavizar uma imagem é utilizando-se um "filtro passa-baixa". Como o próprio nome indica, a característica desse operador é filtrar o sinal preservando apenas os componentes de baixa freqüência. Tal filtro é implementado, a partir de (5.1), por meio do seguinte molde de clonagem 


$$
\bar{B}=\frac{1}{9} \cdot\left(\begin{array}{lll}
1.0 & 1.0 & 1.0 \\
1.0 & 1.0 & 1.0 \\
1.0 & 1.0 & 1.0
\end{array}\right)
$$

que representa a resposta em impulso do filtro. A imagem a ser processada é fixada como a entrada $u$ da rede, e os outros parâmetros são fixados em zero $(A=0$ e $I=0$ em (5.1)). O efeito produzido por esse filtro é efetuar uma média da intensidade dos pixels de uma vizinhança, como mostra a máscara $\bar{B}$. Assim, é de se esperar que a imagem resultante seja mais suave, pois cada pixel carregará a informação dos pixels vizinhos (ambos estarão correlacionados), diferentemente do que acontece quando existe ruído. Na Seção 5.5, é apresentada uma simulação utilizando esse tipo de filtro. 


\subsubsection{Extração de contornos}

Sabe-se que os contornos em imagens retém informação essencial para a compreensão do conteúdo das mesmas [Lim, 1990]. Dessa forma, muitos algoritmos que realizam o processamento de imagem nas fases de mais alto nível (segmentação baseada em forma, por exemplo) requerem, como entrada, imagens com os contornos ressaltados, para facilitar o reconhecimento das mesmas. Portanto, a extração de contornos é importante em um sistema de visão computacional.

A imagem a ser processada pode ser vista como uma função $I: D \rightarrow C\left(D \subset \mathbb{Z}^{2}, C \subset \mathbb{Z}\right)$, que associa a cada pixel $(i, j) \in D$ da imagem sua informação de cor (intensidade) $I(i, j) \in C$. Cada pixel, por sua vez, pode ser associado a uma célula da RNC, sendo que a intensidade dos mesmos é representada pelo estado da célula subjacente. Para descrever o problema de extração de contornos, no entanto, ao invés de se considerar uma função $I(i, j)$, seja $F: R^{2} \rightarrow R$ uma função contínua que represente a imagem (a função $I(i, j)$ é considerada uma discretização de $F(x, y)$ ). Como os contornos em uma imagem são identificados por mudanças bruscas na intensidade dos pixels, tanto o cálculo do gradiente, quanto o cálculo da segunda derivada da imagem, provém informações acerca de pontos referentes a contornos. Assim, pontos $(x, y)$ pertencentes a contornos são tais que

(a) o módulo do gradiente $|\nabla F(x, y)|$ assume valores altos, o que indica um variação brusca na intensidade dos pixels, em relação aos vizinhos;

(b) o módulo das derivadas de segunda ordem de $F(x, y)$ é nulo, o que indica pontos de inflexão, que correspondem a extremos do gradiente de $F(x, y)$.

O gradiente $\nabla F(x, y)$ de $F$ é definido como:

$$
\nabla F(x, y)=\left(\frac{\partial F(x, y)}{\partial x}, \frac{\partial F(x, y)}{\partial y}\right)
$$

cujo módulo

$$
\|\nabla F(x, y)\|=\sqrt{\left(\frac{\partial F}{\partial x}\right)^{2}+\left(\frac{\partial F}{\partial y}\right)^{2}}
$$

pode ser aproximado por

$$
\|\nabla F(x, y)\| \approx\left|\frac{\partial F}{\partial x}\right|+\left|\frac{\partial F}{\partial y}\right|,
$$

por conveniência computacional (facilidade de cálculo). Existem diversas formas de discretizar as derivadas parciais [Press et al., 1993], sendo aqui utilizada a fórmula 


$$
\begin{aligned}
& \frac{\partial F}{\partial x} \approx(1 / \delta h)(I(i+\delta h, j)-I(i, j)) \\
& \frac{\partial F}{\partial y} \approx(1 / \delta h)(I(i, j+\delta h)-I(i, j))
\end{aligned}
$$

onde $\delta h=1$ é o passo de discretização (corresponde à distância de um pixel) e $I(i, j)$ é a discretização da função $F(x, y)$ que representa a imagem. O cálculo do módulo do gradiente é então implementado pelo seguinte molde de clonagem:

$$
\bar{B}=\left(\begin{array}{rrr}
0.0 & 1.0 & 0.0 \\
0.0 & -2.0 & 1.0 \\
0.0 & 0.0 & 0.0
\end{array}\right)
$$

A imagem $I(i, j)$, a ser processada, é usada como entrada $u_{i j}$ da rede, e os outros parâmetros em (5.1) são fixados em zero $(A=0$ e $I=0)$. Com essa configuração de parâmetros, a evolução do estado da rede no tempo, corresponderá ao cálculo do gradiente da imagem presente no parâmetro $u$ da rede. Os pixels de contorno, que correspondem a valores altos para o módulo do gradiente da imagem, serão ressaltados, como mostram as simulações na próxima seção.

A extração de contornos pelo cálculo do módulo das derivadas segundas de $F(x, y)$, pode ser feita pelo operador Laplaciano (definido na Seção 5.4.2):

$$
\Delta^{2} F=\frac{\partial^{2} F(x, y)}{\partial x^{2}}+\frac{\partial^{2} F(x, y)}{\partial y^{2}}
$$

que é discretizado como em (5.6), e implementado pelo molde de clonagem $\bar{B}$ :

$$
\bar{B}=\left(\begin{array}{rrr}
0.0 & 1.0 & 0.0 \\
1.0 & -4.0 & 1.0 \\
0.0 & 1.0 & 0.0
\end{array}\right)
$$

A imagem a ser processada é então introduzida na entrada $u$ da RNC, e os outros parâmetros são fixados em zero $(A=0$ e $I=0)$. Nesse caso, a evolução da rede corresponderá ao cálculo da magnitude das derivadas de segunda ordem da imagem. Como os pixels do contorno correspondem a pontos de inflexão (onde ocorre troca de sinal da derivada segunda), estes serão ressaltados. Por outro lado, pixels onde não ocorre variação de intensidade dentro da vizinhança considerada, terão atribuídos um valor de intensidade igual a zero. Pode-se perceber que o operador $\bar{B}$ calcula a diferença entre a intensidade do pixel de referência (elemento central) e a dos quatro outros vizinhos e, se essa diferença for grande, isso denota um ponto do contorno, como também um ruído. Por esse motivo, é sempre adequado suavizar uma imagem, antes de extrair seus contornos. 


\subsection{Simulações}

Nas simulações realizadas, cada pixel da imagem processada é associado a uma célula da RNC. No primeiro exemplo, a imagem é usada como a condição inicial $x(0)$ da rede. No segundo exemplo, a imagem é utilizada como a entrada $u$ da rede. Em ambos os casos, a saída $y(t)$, quando $t \rightarrow \infty$, representa a imagem final processada.

Como a faixa de operação inicial de $x_{i j}(0)$ é limitada em $[-1.0,1.0]$, por (5.3), esse intervalo é utilizado para codificar as cores dos pixels da imagem processada. As imagens processadas foram representadas em uma escala de tons de cinza, sendo que o valor -1.0 foi usado para codificar a cor branca e o valor 1.0 a cor preta. Valores intermediários nesse intervalo denotam os demais tons na escala de cinza.

Também, em ambas simulações, foi utilizada uma vizinhança de $3 \times 3$, que corresponde ao parâmetro $r=1$ em (5.1). As equações (6.1) foram integradas numericamente, pelo algoritmo Runge-Kutta de quarta ordem [Press et al., 1993].

\subsubsection{Simulação 1: Remoção de ruído}

Para demonstrar a remoção de ruído por uma RNC, a imagem original na Figura 5.3(a) foi corrompida com $10 \%$ de ruído gaussiano (desvio padrão $\sigma=1$ ), como mostra a Figura 5.3(b). Na Figura 5.3(c), por sua vez, vê-se o resultado da suavização, utilizando-se o operador Laplaciano, como especificado na Seção 5.4.2. Alternativamente, quando um filtro passa-baixa é utilizado para remoção de ruídos, a saída processada é aquela mostrada na Figura 5.3(d).

\subsubsection{Simulação 2: Extração de contornos}

A mesma imagem da Figura 5.3(a), é agora utilizada para exemplificar a extração de contornos por uma RNC. Na Figura 5.4(a), vê-se o resultado da extração de contornos com o cálculo baseado no módulo do gradiente da imagem, como discutido na Seção 5.4.3. Na Figura 5.4(b), por sua vez, tem-se o resultado dessa mesma tarefa, com a aplicação do operador Laplaciano.

Como é usual em processamento de imagens, o resultado da extração de contornos é uma imagem binária, onde a cor preta denota o plano de fundo e a cor branca os contornos detectados. Para que se consiga esse mesmo resultado com uma RNC, é necessário utilizar o parâmetro $I$ com um valor diferente de zero. Fixando-se $I=-0.7$, por exemplo, o resultado da operação de extração de contornos será como mostra as Figuras 5.4(c) e 5.4(d), onde a cor preta equivale a uma saída igual a 1 , e a cor branca a um valor igual a -1 . O parâmetro $I$, portanto, funciona como um deslocamento que é introduzido no estado das células, controlando a intensidade dos pixels na imagem de saída da rede. 


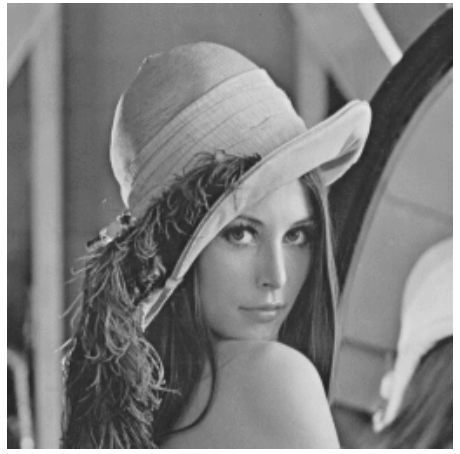

(a)

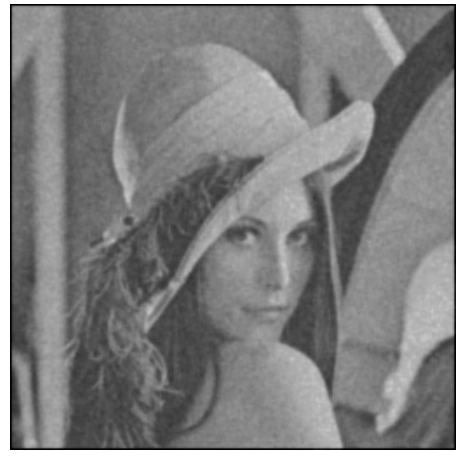

(c)

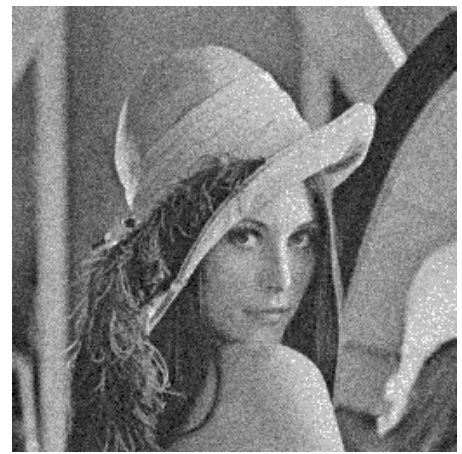

(b)

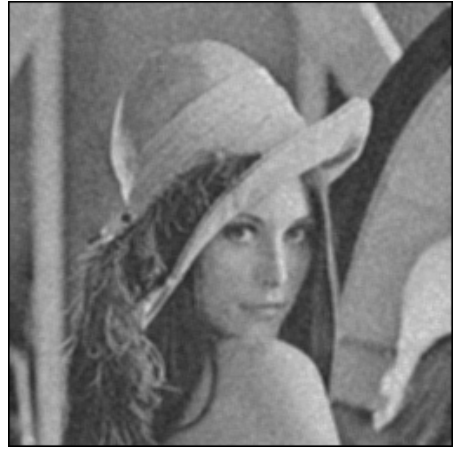

(d)

Figura 5.3: (a) imagem original de entrada, em $t=0$; (b) imagem corrompida com $10 \%$ de ruído gaussiano ( $\sigma=1)$; (c) suavização pelo operador Laplaciano; (d) suavização por filtro passa-baixa.

\subsection{Discussão}

Em [Werblin et al., 1995], discute-se a construção de um "olho biônico", cuja estrutura subjacente é uma RNC programada para o processamento de imagens. As RNCs tem um alto potencial para esse domínio de aplicação, pois, quando implementadas em hardware, possibilitam processamento de baixo-nível, em tempo real. As características verificadas no modelo de rede neural considerado indicam que o mesmo se mostra adequado para tarefas que envolvam cálculos ou processamentos "locais" intensivos.

De maneira geral, os parâmetros de uma RNC podem ser escolhidos de acordo com heurísticas obtidas em certo domínio de aplicação, ou por algoritmos de aprendizado, como em outros modelos de redes neurais. Como exemplo, foi demonstrada a aplicação de RNCs para o processamento de imagens, fazendo-se a escolha de seus parâmetros por meio de heurísticas nesse domínio. No próximo capítulo é demonstrado como RNCs podem ser programadas por algoritmos de aprendizado, de modo que funcionem como memórias associativas para o armazenamento e recuperação de padrões. 


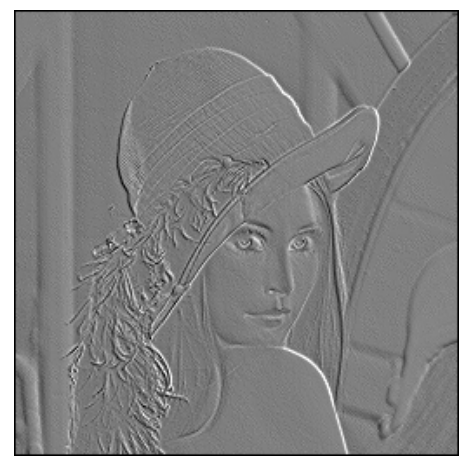

(a)

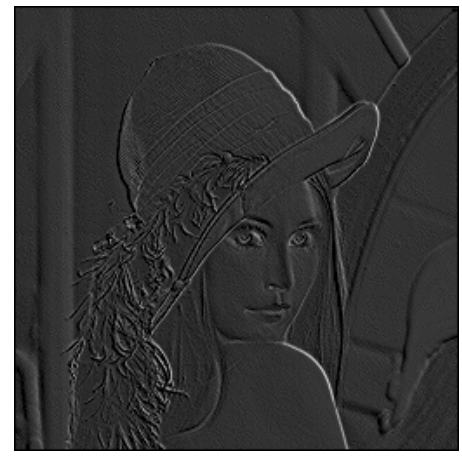

(c)

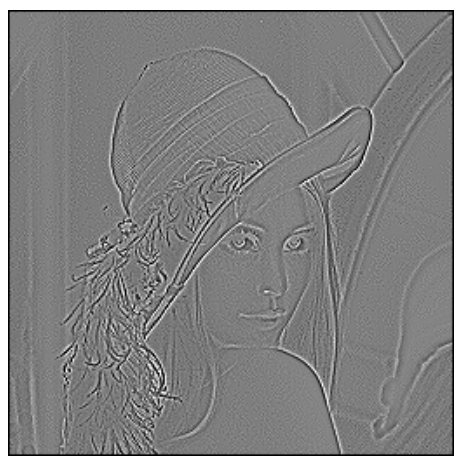

(b)

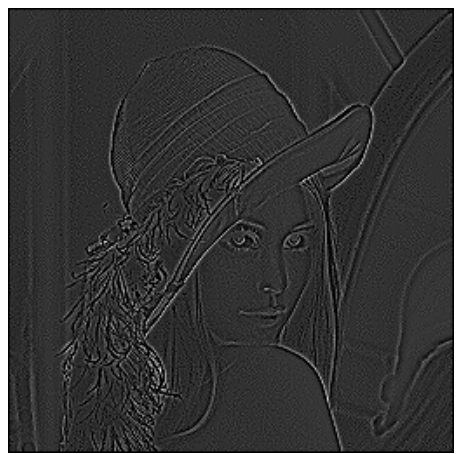

(d)

Figura 5.4: (a) Extração de contornos pelo cálculo do gradiente; (b) Extração de contornos pelo operador Laplaciano; (c) Contornos obtidos com o cálculo do gradiente, após modificação; (d) Contornos obtidos com o operador Laplaciano, após modificação. 


\section{Capítulo 6}

\section{Memória associativa em RNCs}

Como visto no modelo de Hopfield, o objetivo de uma memória associativa é armazenar e recuperar informações, com base no conteúdo das mesmas. Nesse capítulo, investiga-se como uma RNC pode ter seus parâmetros ajustados, para que funcione como uma memória associativa.

Uma memória associativa deve apresentar as seguintes propriedades, que serão investigadas nos métodos apresentados a seguir:

- Ter alta capacidade de completar padrões corrompidos por ruído;

- Apresentar baixo número de estados espúrios;

- Ter alta capacidade de armazenamento.

Os métodos de construção de memória associativa em RNCs, apresentados a seguir, são comparados com base no nível de atendimento a esses critérios, visto que, na prática, nem todos são possíveis de serem realizados, ou são realizáveis em maior ou menor grau, dependendo das características do método estudado. Tal comparação é apresentada no Capítulo 7.

\subsection{Formulação do problema}

As equações que descrevem uma RNC (5.1) podem ser colocadas na forma vetorial (caso contínuo), como visto na Seção 5.2:

$$
\begin{gathered}
\dot{x}=-x+T y+I \\
y=\operatorname{sat}(x)
\end{gathered}
$$

onde $x(t) \in \mathbb{R}^{n}, T \in \mathbb{R}^{n \times n}, y \in \mathbb{D}^{n}\left(D^{n}=\left\{y \in \mathbb{R}^{n}:-1 \leq y_{i} \leq 1, i=1, \ldots, n\right\}\right)$, $\operatorname{sat}(x)=\left[\operatorname{sat}\left(x_{1}\right), \ldots, \operatorname{sat}\left(x_{n}\right)\right]^{T}, \operatorname{sat}\left(x_{i}\right)$ é a função saturação (equação (5.2)), $I \in \mathbb{R}^{n}$, e $n$ é o número de células da rede. 
A saída da rede, representada pela variável $y$ em (6.1), deve corresponder aos padrões de memória a serem armazenados. Assim, os pontos de equilíbrio devem ser apropriadamente escolhidos para resultar na saída desejada da rede. Como pontos de equilíbrio são tais que:

$$
\dot{x}=0
$$

a condição para existência destes em (6.1) é dada por

$$
\begin{aligned}
x^{p} & =T y^{p}+I, \quad p=1, \ldots, m \\
y^{p} & =\operatorname{sat}\left(x^{p}\right)
\end{aligned}
$$

onde $x^{p} \in \mathbb{R}^{n}, p=1, \ldots, m$ denotam os pontos de equilíbrio do sistema e $y^{p} \in B^{n}\left(B^{n}=\right.$ $\left\{y \in \mathbb{R}^{n}: y_{i}=1\right.$ ou $\left.y_{i}=-1, i=1, \ldots, n\right\}$ ) as saídas da rede (que correspondem aos ítens de memória a serem armazenados). Note que os padrões de memória $y^{p}$ são restritos a padrões binários $\left(y^{p} \in B^{n}\right)$, pois essa condição garante uma maior robustez ou capacidade de recuperação dos mesmos. Isto ocorre porque, se $y^{p} \in B^{n}$ e se os equilíbrio forem escolhidos distantes da fronteira de saturação (isto é, se $\left|x^{p}\right| \gg 1$ ), para pequenas variações nesses, as saídas $y^{p}$ não são alteradas (pois $y^{p}=\operatorname{sat}\left(x^{p}\right)$ ).

Os métodos de síntese de memória associativa, apresentados nas seções seguintes, fazem a escolha dos pontos de equilíbrio $x^{p}$ de (6.2) como argumentado, buscando uma maior robustez na recuperação dos padrões. Tal argumentação é formalizada pelo Corolário B.3.3 (vide Apêndice B), que dá condições acerca da estabilidade assintótica dos pontos de equilíbrio mencionados. Os métodos apresentados diferem basicamente nas estratégias tomadas para determinar os parâmetros livres $T$ e $I$ em (6.2), dados os $m$ padrões de treinamento (ítens de memória) $y^{p}, p=1, \ldots, m$ a serem armazenados.

Na exposição dos métodos que seguem, considere os seguintes conjuntos:

$$
B^{n}=\left\{x \in \mathbb{R}^{n}: x_{i}=1 \text { ou } x_{i}=-1, i=1, \ldots, n\right\},
$$

formado por vetores cujos componentes são binários e, para cada $y \in B^{n}$, seja

$$
C(y)=\left\{x \in \mathbb{R}^{n}: x_{i} y_{i}>1, i=1, \ldots, n\right\}
$$

o conjunto formado por vetores de componentes de mesmo sinal que os componentes binários do vetor $y$, e magnitude maior que 1 . 


\subsection{Método baseado em Matriz Pseudo-Inversa}

Esse método foi proposto em [Grassi, 2001]. Inicialmente, para simplificar o problema, suponha que a rede é totalmente conectada, isto é, não existe nenhuma restrição sobre a matriz de conexões $T$. A condição de existência de pontos de equilíbrio na dinâmica da RNC é, de acordo com (6.2):

$$
\begin{aligned}
x^{p} & =T y^{p}+I, \quad p=1, \ldots, m \\
y^{p} & =\operatorname{sat}\left(x^{p}\right)
\end{aligned}
$$

onde $y^{p} \in B^{n}$ são vetores binários que correspondem aos padrões a serem armazenados. Como $y^{p}=\left[\operatorname{sat}\left(x_{1}^{p}\right), \ldots, \operatorname{sat}\left(x_{n}^{p}\right)\right]^{T}$, para $p=1, \ldots, m$, sabe-se que se os vetores $x^{p}$ em (6.3) tiverem componentes de mesmo sinal que $y^{p}$ e magnitude maior que 1 , os vetores $y^{p}$ serão armazenados como memórias de (6.1) (pelo Corolário B.3.3). Esta condição é equivalentemente representada por

$$
x^{p}=\alpha y^{p}, \quad p=1, \ldots, m
$$

para algum $\alpha>1$.

A equação (6.3) pode ser colocada na forma matricial:

$$
X=T Y+\bar{I}
$$

onde $Y=\left[y^{1}, \ldots, y^{m}\right] \in \mathbb{R}^{n \times m}, X=\alpha Y \in \mathbb{R}^{n \times m}, \alpha>1$ e $\bar{I}=[I, \ldots, I] \in \mathbb{R}^{n \times m}$.

Seja

$$
\begin{aligned}
& R=\left[Y^{T}, J\right] \in \mathbb{R}^{m \times(n+1)}, \\
& W=[T, I] \in \mathbb{R}^{n \times(n+1)}, \\
& J=[1,1, \ldots, 1]^{T} \in \mathbb{R}^{m} .
\end{aligned}
$$

A equação (6.4) pode então ser reescrita como

$$
X^{T}=R W^{T}
$$

Sejam $X_{i} \in \mathbb{R}^{1 \times m}$ e $W_{i} \in R^{1 \times n+1}$ as $i$-ésimas linhas de $X$ e $W$, respectivamente. Assim, a equação anterior pode ser reescrita como

$$
X_{i}^{T}=R W_{i}^{T}, \quad i=1, \ldots, n
$$

Sabe-se que (6.6) só possui solução se o vetor $X_{i}^{T}$ for uma combinação linear das colunas de $R$ (com escalares dados pelos componentes de $W_{i}^{T}$ ), o que equivale a dizer que só é possível 
encontrar uma solução se $R$ for invertível (para maiores detalhes, ver [Lay, 1996]). Entretanto, é necessário determinar $W_{i}^{T}$, na equação (6.6), mesmo que $R$ não seja invertível. O que se faz, nesse caso, é encontrar uma solução que sempre exista e que seja ótima, no sentido de minimizar

$$
\left\|X_{i}^{T}-R W_{i}^{T}\right\|, \quad i=1, \ldots, n .
$$

onde $\|\cdot\|$ denota a norma Euclidiana. Tal solução, portanto, é dita minimizar o erro quadrático da expressão acima, sendo calculada por

$$
\hat{W}_{i}^{T}=R^{+} X_{i}^{T}, \quad i=1, \ldots, n
$$

onde $R^{+}$denota a matriz pseudo-inversa de $R$, que é definida como

$$
R^{+}=\left(R^{T} R\right)^{-1} R^{T}
$$

caso $\left(R^{T} R\right)$ seja invertível. Caso contrário, $R^{+}$pode ser obtida por meio da fatoração SVD (ver Apêndice C), por exemplo. Se $R$ é invertível, então $R^{+}=R^{-1}$ e a solução (6.7) é, de fato

$$
W_{i}^{T}=R^{-1} X_{i}^{T}, \quad i=1, \ldots, n .
$$

Em termos geométricos, a solução dada por $\hat{W}_{i}^{T}$ em (6.7), projeta o vetor $X_{i}^{T}$ no subespaço gerado pelas colunas de $R$ (denotado por $C o l R$ ), como ilustra a Figura 6.1.

\subsubsection{Matriz de índice e restrição de conectividade de RNCs}

Como uma RNC é uma rede localmente acoplada, apenas alguns elementos de $T$ podem ser utilizados para a conexão entre as células da rede, sendo que os outros elementos devem ser fixados em zero. No entanto, tal restrição não foi utilizada para obter a solução dada por (6.7). Esta restrição pode ser agora introduzida na formulação do problema, por meio das definições que seguem.

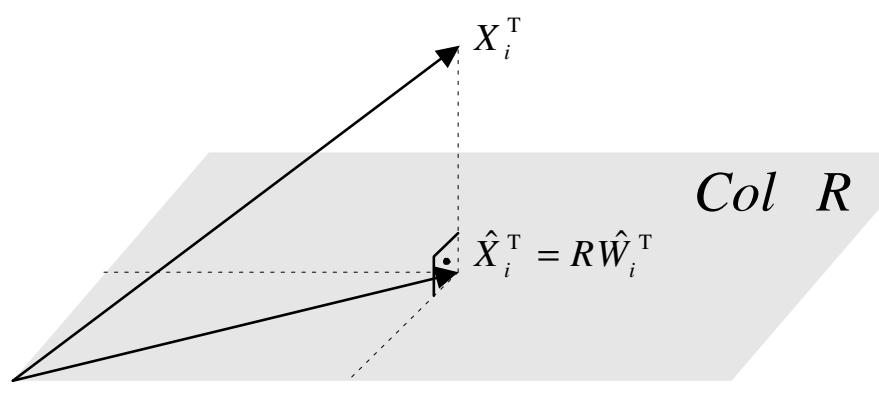

Figura 6.1: Projeção de $X_{i}^{T}$ no subespaço $C o l R$. 
Definição 6.2.1 Uma matriz $S=\left[S_{i j}\right] \in \mathbb{R}^{n \times n}$ é dita uma matriz de índice se

$$
S_{i j}=0 \text { ou } 1, \quad \text { para } i, j=1, \ldots, n \text {. }
$$

Observação 6.2.2 Uma matriz de índice $S=\left[S_{i j}\right]$ codifica a arquitetura da rede, indicando a existência $\left(S_{i j}=1\right)$ ou não $\left(S_{i j}=0\right)$ de uma interconexão entre a célula de índice i e a célula de indice $j$ da rede.

A seguir, define-se como uma matriz $S$ pode ser utilizada para restringir as conexões de uma RNC.

Definição 6.2.3 Seja $S$ como na Definição 6.2.1, e seja $W=\left[W_{i j}\right] \in \mathbb{R}^{n \times n}$. A restrição de uma matriz de índice $S$ sobre $W$ é denotada por

$$
W \mid S=\left[h_{i j}\right]
$$

onde

$$
h_{i j}=\left\{\begin{array}{cc}
W_{i j} & , \text { se } S_{i j}=1 \\
0 & , \text { se } S_{i j}=0
\end{array}\right.
$$

A partir dessas definições, o problema de construção da memória associativa é reformulado e resolvido na seção seguinte, considerando-se a restrição de conectividade local da rede.

\subsubsection{Estratégia de resolução revista}

A equação (6.4), com a restrição de uma matriz de índice $S$ sob a matriz de conexões $T$ (isto é, $T \mid S)$, pode ser reformulada como

$$
X=\bar{T} Y+\bar{I}
$$

onde $\bar{T}=T \mid S$. Esta equação, por sua vez, é equivalente a

$$
X_{i}=\bar{T}_{i} Y+\bar{I}_{i}, \quad i=1, \ldots, n
$$

onde $X_{i} \in \mathbb{R}^{1 \times m}, \bar{T}_{i} \in \mathbb{R}^{1 \times n}$ e $\bar{I}_{i} \in \mathbb{R}^{1 \times m}$ denotam as $i$-ésimas linha de $X, \bar{T}$ e $\bar{I}$, respectivamente. 
Devido à restrição $T \mid S$, a matriz $\bar{T}$ é possivelmente esparsa, isto é, contém vários elementos iguais a zero e, conseqüentemente, os elementos de $Y$ que são multiplicados por essas posições nulas, podem também ser descartados da equação. Considere a $i$-ésima linha de $\bar{T}$, denotada por $\bar{T}_{i}$. Eliminando-se as linhas de $Y$ que são multiplicadas por zeros em $\bar{T}_{i}$, isto é, as $j$-ésimas linhas de $Y$ para as quais $S_{i j}=0$, para um $i$ fixo, obtém-se a matriz $\tilde{Y}_{i} \in \mathbb{R}^{v_{i} \times m}$, onde $v_{i}$ indica a quantidade de elementos não-nulos em $\bar{T}_{i}$ (a quantidade de vizinhos da célula $i$ ), dada por

$$
v_{i}=\sum_{j=1}^{n} S_{i j} .
$$

Eliminando-se, também, os componentes do vetor $\bar{T}_{i}$ iguais a zero, isto é, os $j$-ésimos componentes para os quais $S_{i j}=0$, para um $i$ fixo, obtém-se o vetor $\tilde{T}_{i} \in \mathbb{R}^{1 \times v_{i}}$. Assim, (6.9) pode ser reescrita como

$$
X_{i}=\tilde{T}_{i} \tilde{Y}_{i}+\bar{I}_{i}, \quad i=1, \ldots, n
$$

Defina agora

$$
\begin{aligned}
& \tilde{R}_{i}=\left[\tilde{Y}_{i}^{T}, J\right] \in \mathbb{R}^{m \times\left(v_{i}+1\right)}, \\
& J=[1, \ldots, 1]^{T} \in R^{m}, \\
& \tilde{W}_{i}=\left[\tilde{T}_{i}, I_{i}\right] \in R^{1 \times\left(v_{i}+1\right)} .
\end{aligned}
$$

Assim, de (6.11), resulta

$$
X_{i}^{T}=\tilde{R}_{i} \tilde{W}_{i}^{T}, \quad i=1, \ldots, n
$$

cuja solução, utilizando matrizes pseudo-inversas, é

$$
\hat{W}_{i}^{T}=\tilde{R}_{i}^{+} X_{i}^{T}, \quad i=1, \ldots, n
$$

Dessa forma, foi obtida uma solução para o problema com restrições sobre a estrutura de $T$ (equação (6.8)) da mesma forma como foi obtida uma solução para (6.3), graças à eliminação das posições não permitidas em $T$ e, consequientemente, em $Y$, de acordo com a restrição de conectividade local de RNCs.

Observação 6.2.4 Para se "reconstruir" a matriz T em (6.4), a partir da solução $\hat{W}_{i}$ em (6.12), aplica-se o seguinte procedimento: o primeiro componente do vetor $\hat{W}_{i}$, denotado por $\hat{W}_{i}(1)$, é atribuído ao primeiro componente de $T_{i}$, tal que $S_{i j}=1$. O segundo componente $\hat{W}_{i}(2)$, é atribuído ao segundo componente de $T_{i}$, tal que $S_{i j}=1$, e assim sucessivamente, para os $v_{i}$ componentes de $\hat{W}_{i}$. A última posição do vetor $\hat{W}_{i}$, denotada por $\hat{W}_{i}\left(v_{i}+1\right)$, corresponde ao valor do parâmetro $I_{i}$, isto é, $I_{i}=\hat{W}_{i}\left(v_{i}+1\right)$. Este algoritmo é repetido para cada linha $T_{i}$ de 
$T$ e para cada componente $I_{i}$ de $I$, para $i=1, \ldots n$. Os elementos de $T_{i}$ que não tiveram um valor atribuído, correspondem àqueles que não pertencem à vizinhança da i-ésima célula da rede e são, portanto, igualados a zero.

O método para determinação dos parâmetros da RNC descrito, é sintetizado pelo Algoritmo 6.2.1.

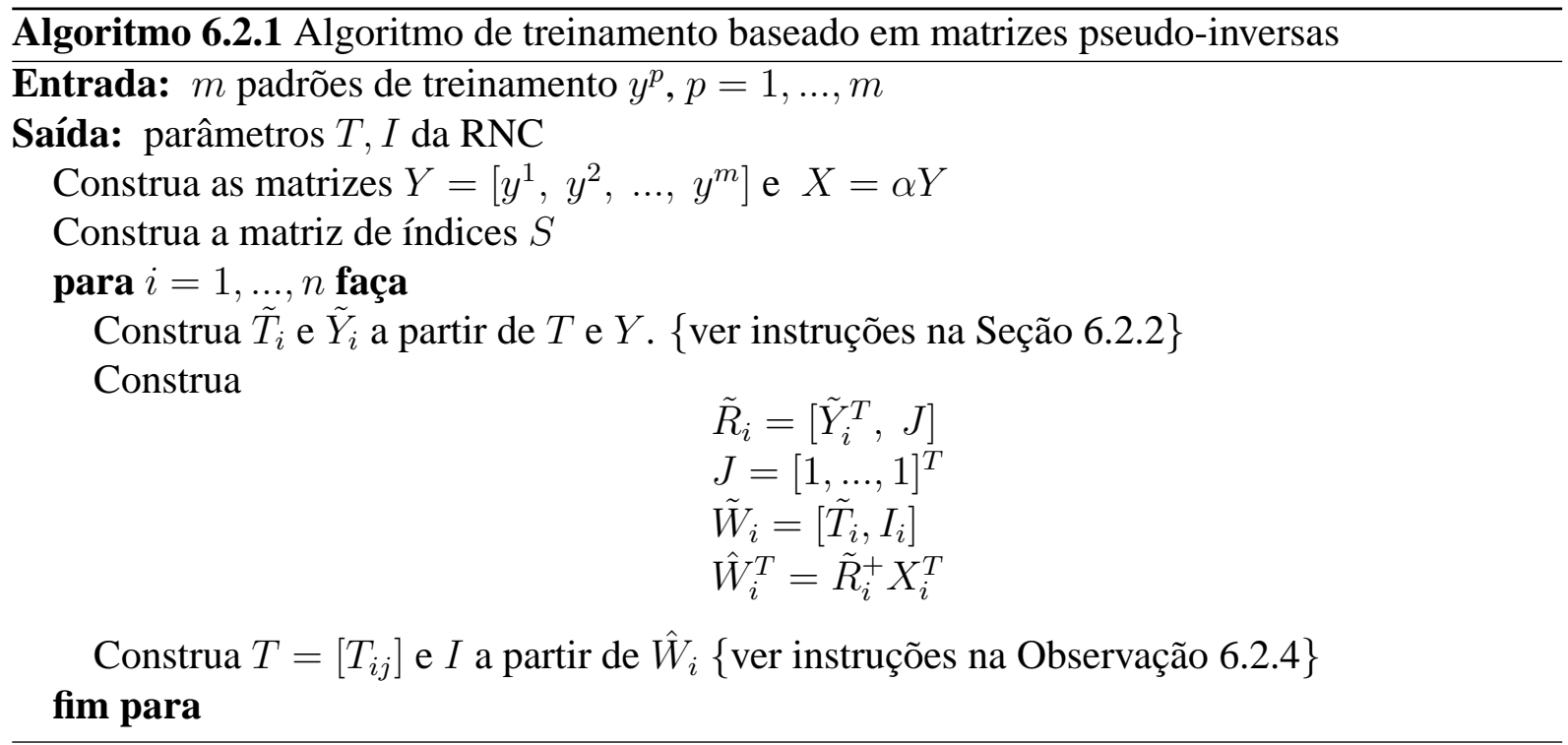




\subsection{Método baseado em Decomposição por Valor Singular}

Neste método, proposto por Liu e Michel [Liu \& Michel, 1994], obtém-se uma solução para a equação (6.2) essencialmente como no método anterior, por matrizes pseudo-inversas, mas agora calculadas por uma Decomposição por Valor Singular (ver Apêndice C). Uma possível forma de resolver o sistema de equações (6.2) é atribuindo-se um valor para $T$ de maneira que, em seguida, $I$ seja calculado em função desse $T$ escolhido. Considere $T$ obtido a partir de

$$
x^{p}-x^{m}=T\left(y^{p}-y^{m}\right), \quad p=1, \ldots,(m-1) .
$$

Pode-se calcular $I$ em função de $T$ assim obtido, por:

$$
I=x^{m}-T y^{m}
$$

Essa estratégia permite obter $T$ e $I$ que satisfazem as equações de ponto de equilíbrio. Para verificar isso, considere o lado direito de (6.2). Para $y^{p}, p=1, \ldots, m$ tem-se que

$$
\begin{aligned}
T y^{p}+I & =T y^{p}+x^{m}-T y^{m}= \\
& =T\left(y^{p}-y^{m}\right)+x^{m}= \\
& =x^{p}-x^{m}+x^{m}= \\
& =x^{p}
\end{aligned}
$$

o que verifica, portanto, a equação (6.2).

Sendo que a matriz $T$ é obtida a partir da solução de (6.13), cabe observar que $T$ deve ser tal que $T \mid S$ (ver Definição 6.2.3), para uma matriz de índice $S$. Considerando essa restrição, é possível reescrever (6.13) na forma matricial:

$$
X=\bar{T} Y
$$

onde $\bar{T}=T \mid S, Y=\left[y^{1}-y^{m}, y^{2}-y^{m}, \ldots, y^{(m-1)}-y^{m}\right] \in \mathbb{R}^{n \times(m-1)}$ e $X=\alpha Y \in \mathbb{R}^{n \times(m-1)}$, para algum $\alpha>1$. Como se deseja obter uma solução de (6.2) somente para os elementos de $\bar{T}$ diferentes de zero, faz-se necessário reescrever a equação anterior, eliminando os elementos nulos das matrizes. Como a quantidade de zeros em cada linha de $\bar{T}$ pode diferir, a equação (6.15) é reescrita da seguinte forma:

$$
X_{i}=\bar{T}_{i} Y, \quad i=1, \ldots, n
$$

onde $\bar{T}_{i} \in \mathbb{R}^{1 \times n}$ denota a $i$-ésima linha de $\bar{T}$ e $X_{i} \in \mathbb{R}^{1 \times(m-1)}$ denota a $i$-ésima linha de $X$. Eliminando-se, então, os elementos nulos de $\bar{T}_{i}$, como discutido na Seção 6.2.2, obtém-se um vetor denotado por $\tilde{T}_{i} \in \mathbb{R}^{1 \times v_{i}}$, onde $v_{i}$ é dado por (6.10). De maneira similar para $Y$, obtém-se 
uma matriz $\tilde{Y} \in \mathbb{R}^{v_{i} \times(m-1)}$, pela eliminação das linhas que são multiplicadas por elementos nulos de $\bar{T}_{i}$. Assim, pode-se reescrever (6.16) como

$$
X_{i}=\tilde{T}_{i} \tilde{Y}_{i}, \quad i=1, \ldots, n
$$

cuja solução, dada em termos de matrizes pseudo-inversas, é

$$
\hat{T}_{i}=X_{i}\left(\tilde{Y}_{i}\right)^{+}
$$

No método discutido, utiliza-se uma versão modificada dessa solução por pseudo-inversa, dada por:

$$
\bar{T}_{i}=X_{i}\left(\tilde{Y}_{i}\right)^{+}+w_{i} U_{i 2}^{T}, \quad i=1, \ldots, n
$$

onde $w_{i} \in R^{1 \times v_{i}}$ é um vetor arbitrário, e $U_{i 2}$ é obtida por uma Decomposição por Valor Singular (ver Apêndice C) de $\tilde{Y}_{i}$ :

$$
\tilde{Y}_{i}=\left[U_{i 1} \mid U_{i 2}\right] \Sigma_{i}\left[\frac{V_{i 1}^{T}}{V_{2}}\right] .
$$

É possível mostrar que mesmo com à adição do termo $w_{i} U_{i 2}^{T}$ à solução por pseudo-inversa (equação (6.19)), a solução resultante verifica (6.13) [Liu \& Michel, 1994].

Observação 6.3.1 As linhas da matriz $T$ em (6.2) são construídas a partir dos vetores $\bar{T}_{i}$, obtidos em (6.19), de forma análoga à apresentada na Observação 6.2.4 (ver Seção 6.2.2).

A partir da matriz $T$ reconstruída com os vetores obtidos da solução (6.19), os elementos de $I$ são calculador por

$$
I_{i}=x_{i}^{m}-T_{i} y^{m}, \quad i=1, \ldots, n
$$

de acordo com (6.14).

O método de aprendizado considerado nessa seção, é sintetizado pelo Algoritmo 6.3.1, apresentado a seguir.

\subsection{Método baseado em aprendizado Hebbiano}

O método baseado em aprendizado Hebbiano, proposto por Szolgay e colaboradores [Szolgay et al., 1997], escolhe os pontos de equilíbrio $x^{p}, p=1, \ldots, m$ de (6.2) dentro da região de saturação, como nos métodos anteriores. Essa condição é equivalente a 


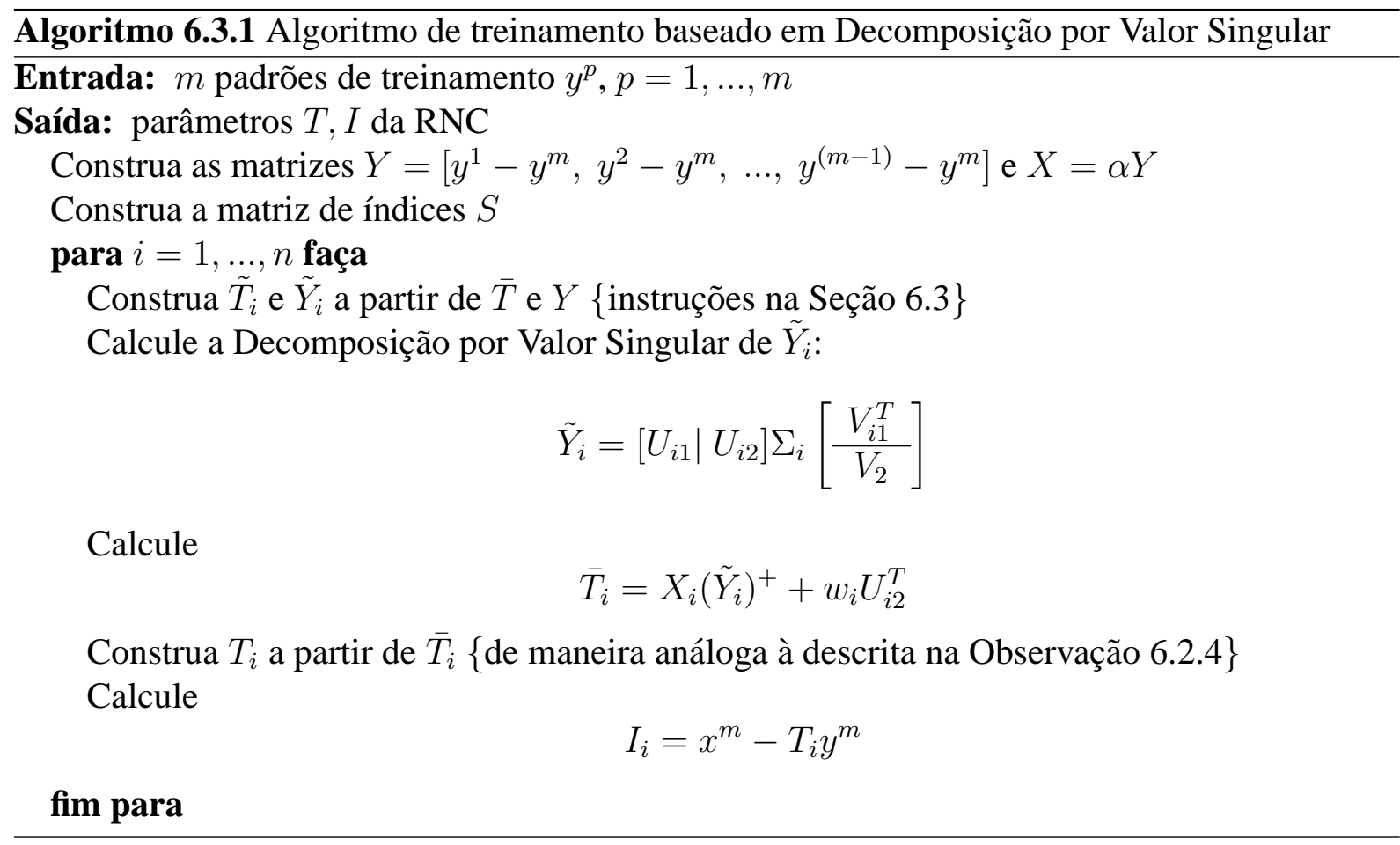

$$
x^{p} \in C\left(y^{p}\right), \quad p=1, \ldots, m
$$

de acordo com o Corolário B.3.3 (ver Apêndice B), onde $y^{p} \in B^{n}$. Assim, pela definição de $C\left(y^{p}\right)$ (ver Seção 6.1):

$$
x_{i}^{p} y_{i}^{p}>1, \quad i=1, \ldots, n \quad e \quad p=1, \ldots, m
$$

onde $x_{i}^{p}$ e $y_{i}^{p}$ denotam os $i$-ésimos componentes dos vetores $x^{p}$ e $y^{p}$, respectivamente. Para conformar com essa notação, as equações de pontos de equilíbrio da RNC (6.2) são reescritas como:

$$
x_{i}^{p}=T_{i}^{T} y^{p}+I_{i}, \quad i=1, \ldots, n \quad e \quad p=1, \ldots, m
$$

onde $T_{i}$ designa a $i$-ésima linha da matriz $T$ e $I_{i}$ o $i$-ésimo componente do vetor $I$. Substituindo a igualdade anterior em (6.20), resulta na condição:

$$
\left(T_{i}^{T} y^{p}\right) y_{i}^{p}+I_{i} y_{i}^{p}>1, \quad i=1, \ldots, n \quad e \quad p=1, \ldots, m .
$$

Considere o vetor $\tilde{T}_{i}$, obtido a partir da eliminação dos componentes de $T_{i}$ iguais a zero, devido à restrição de conectividade local de RNCs (ver Seção 6.2.1). De maneira análoga, considere o vetor $\tilde{y}^{p}$, obtido a partir da eliminação dos componentes de $y^{p}$ que são multiplicados por zeros em $T_{i}$. Então, a inequação (6.21) fica 


$$
\left(\tilde{T}_{i}^{T} \tilde{y}^{p}\right) y_{i}^{p}+I_{i} y_{i}^{p}>1, \quad i=1, \ldots, n \quad e \quad p=1, \ldots, m .
$$

Como é desejável que que os equilíbrios estejam dentro da região de saturação, por questão de robustez na recuperação dos padrões, seja $E>1$ uma constante que determina a magnitude em torno da qual os pontos de equilíbrios vão existir dentro dessa região. A inequação anterior pode ser reescrita como

$$
\left(\tilde{T}_{i}^{T} \tilde{y}^{p}\right) y_{i}^{p}+I_{i} y_{i}^{p}>E>1, \quad i=1, \ldots, n \quad \text { e } \quad p=1, \ldots, m
$$

Considere agora

$$
\begin{aligned}
\bar{T}_{i} & =\left[\tilde{T}_{i}, I_{i}\right] \\
\bar{y}^{p} & =\left[y_{i}^{p} \cdot \tilde{y}^{p}, y_{i}^{p}\right]
\end{aligned}
$$

Da inequação (6.22), tem-se

$$
\bar{T}_{i}^{T} \bar{y}^{p}=E_{i}^{p}>E>1, \quad i=1, \ldots, n \quad e \quad p=1, \ldots, m .
$$

O vetor $\tilde{T}_{i}$ pode então ser obtido da inequação anterior, pela regra de Hebb (ver Seção 4.3):

$$
\tilde{T}_{i}=\sum_{p=1}^{m} w_{i}^{p} y_{i}^{p}, \quad i=1, \ldots, n
$$

onde $w_{i}^{p}$ representa um "fator de ganho" (ou taxa de aprendizado). De acordo com o algoritmo apresentado a seguir, é possível perceber que a condição (6.23) é satisfeita com a escolha de $\tilde{T}_{i}$ feita em (6.24).

Seja $\left[E_{L} ; E_{R}\right]$ um intervalo de variação da magnitude permitida para os pontos de equilíbrio $x^{p}$, em torno $E$, com $E_{L}>1$. O vetor $\bar{T}_{i}$ em (6.23) pode ser obtido de forma iterativa, de acordo com o Algoritmo 6.4.1. 


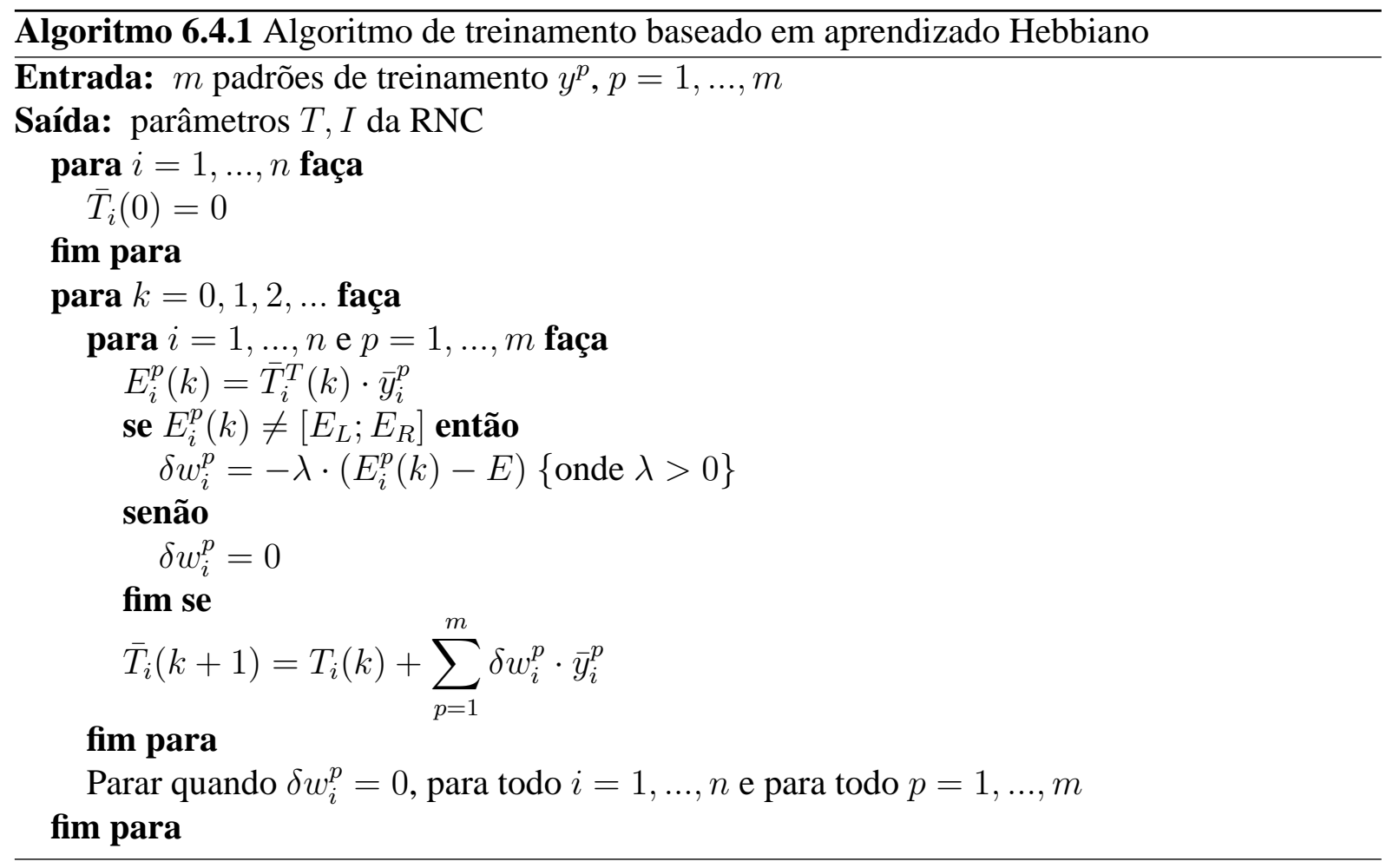

Como se observa no algoritmo, a variação do fator de ganho $\delta w_{i}^{p}$ depende linearmente de $E_{i}^{p}$. A constante $E>1$ é arbitrária, sendo que a obtenção de um valor ótimo é investigada experimentalmente em [Szolgay et al., 1997]. Nessa referência, também é dada a seguinte condição de convergência do algoritmo

$$
0<\lambda<\frac{1}{d \cdot m}
$$

onde $d$ é o número de parâmetros livres (dimensão de $\bar{T}_{i}$ ) a serem determinados, e $m$ é o número de padrões a serem armazenados.

\subsection{Método baseado em aprendizado por Perceptron}

O método apresentado nesta seção, proposto por Liu e Lu [Liu \& Lu, 1997], se utiliza de um Perceptron [Rosenblatt, 1958] para encontrar a matriz de conexões da rede. Na Seção 6.5.1, o conceito de Perceptron é revisto e na Seção 6.5.2, a estratégia de solução que utiliza este conceito é apresentada.

\subsubsection{Perceptrons}

Um Perceptron é uma rede neural estática (ver Seção 3.4), que consiste de neurônios artificiais de McCulloch e Pitts (ver equação (3.1) na Seção 3.2), dispostos em paralelo. É treinado 
para que aprenda a classificar (isto é, distinguir) padrões de entrada entre duas classes préestabelecidas por exemplos (padrões) de treinamento.

Seja $\alpha^{p} \in B^{n}, p=1,2, \ldots, m$ padrões de treinamento, que podem pertencer às classes $X_{1}$ ou $X_{2}$. Um Perceptron deve aprender a classificar corretamente cada padrão de entrada $u=\alpha^{1}, \alpha^{2}, \ldots \alpha^{m}$ em sua respectiva classe, pela fórmula:

$$
z=\operatorname{sgn}(W u)
$$

onde $\operatorname{sgn}(x)=\left[\operatorname{sgn}\left(x_{0}\right), \ldots, \operatorname{sgn}\left(x_{n}\right)\right]$ é a função sinal, definida como:

$$
\operatorname{sgn}\left(x_{i}\right)=\left\{\begin{array}{r}
1, \quad \text { se } x_{i} \geq 0 \\
-1, \quad \text { se } x_{i}<0
\end{array}\right.
$$

Para que o Perceptron aprenda a classificar os padrões de entrada $u \in B^{n}$ em (6.25), deve-se determinar o vetor de pesos $W \in \mathbb{R}^{1 \times n}$ tal que $z=1$, se $u$ pertence a classe $X_{1}$ ou $z=-1$, se $u$ pertence a $X_{2}$. Assim, o aprendizado consiste em resolver um sistema de inequações, de acordo com o algoritmo que segue.

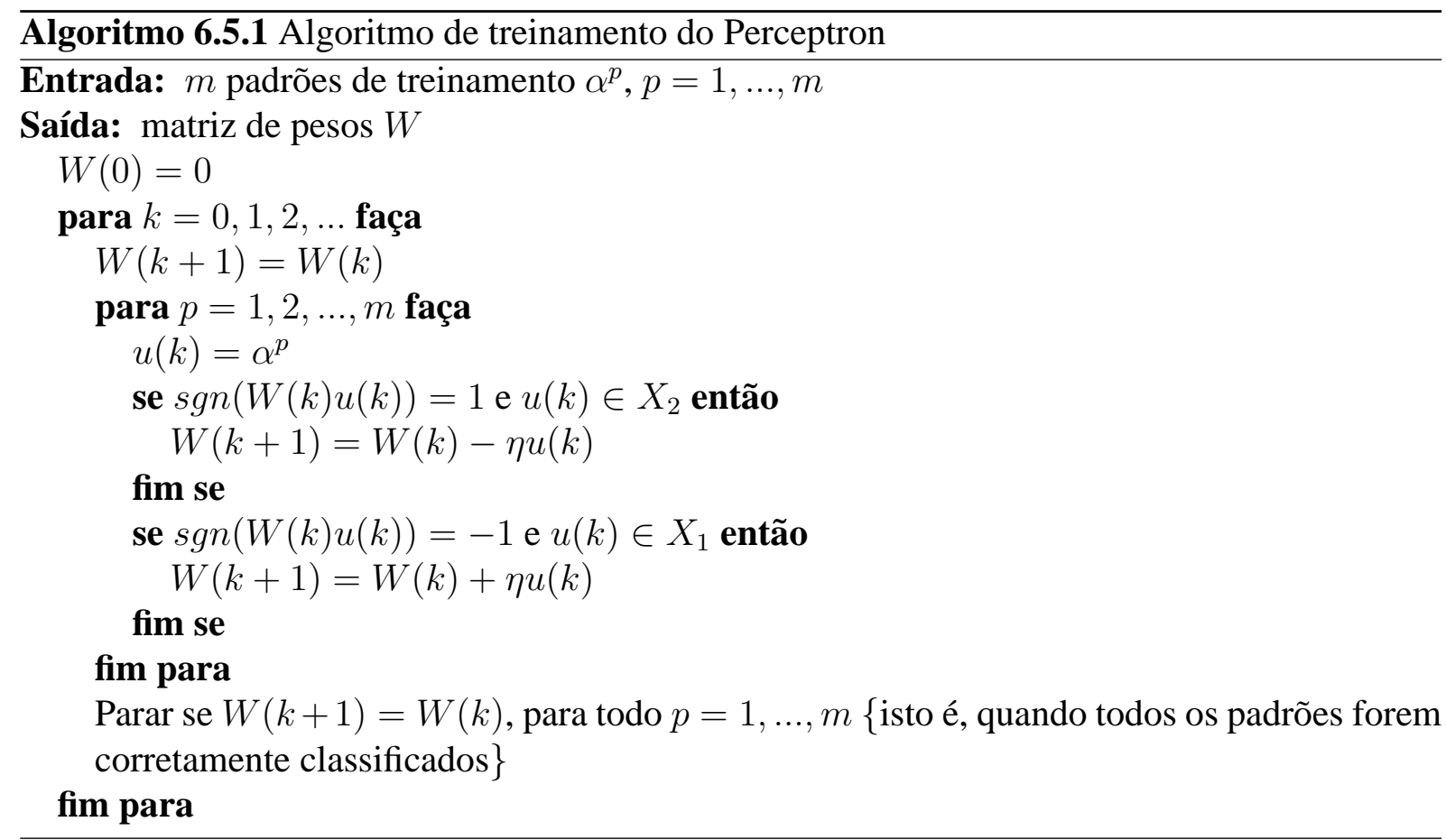

O termo $\eta>0$, utilizado no Algoritmo 6.5.1, controla a taxa de aprendizado do Perceptron. A prova de convergência do algoritmo pode ser encontrada em [Rosenblatt, 1962]. 


\subsubsection{Configuração de RNCs por Perceptrons}

O procedimento utilizado para construção da memória associativa por meio de Perceptrons, consiste em:

- Formular o problema por um sistema de inequações;

- Resolver estas inequações por meio de Perceptrons.

Sabe-se (ver Corolário B.3.3) que a seguinte condição garante o armazenamento dos padrões de memória $y^{p} \in B^{n}, p=1, \ldots, m$ :

$$
x^{p}=T y^{p}+I \in C\left(y^{p}\right), \quad p=1, \ldots, m
$$

que pode ser reescrita, equivalentemente, como:

$$
\begin{cases}T_{i} y_{i}^{p}+I_{i}>1 & \text { se } y_{i}^{p}=1 \\ T_{i} y_{i}^{p}+I_{i}<-1 & \text { se } y_{i}^{p}=-1\end{cases}
$$

para $i=1, \ldots, n$ e $p=1, \ldots, m$, onde $T_{i}$ representa a $i$-ésima linha de $T, I_{i} \mathbf{o} i$-ésimo elemento de $I$ e $y_{i}^{p}$ o $i$-ésimo elemento de $y^{p}$.

A partir de (6.27), explora-se a semelhança dessas inequações com a definição do Perceptron (ver equação (6.25)). Como resultado disso, tem-se o seguinte algoritmo de treinamento de RNCs.

Observação 6.5.1 Notar que o termo $I_{i}$ constitui o elemento $w_{n+1}^{i}$ de $W^{i}$, no Algoritmo 6.5.2, para que a formulação do problema se torne semelhante à formulação do Perceptron (ver equação (6.25)). A adição do termo $\mu_{i}>1$, por sua vez, assegura que (6.27) é satisfeita (a prova pode ser encontrada em [Liu \& Lu, 1997]).

\subsection{Método baseado em Inequações Matriciais Lineares}

Park e colaboradores [Park et al., 2001], em seu método de treinamento de RNCs, formulam o problema de armazenamento dos padrões como um problema de otimização. Sabe-se, pelo Corolário B.3.3 (ver Apêndice B), que a seguinte condição garante o armazenamento dos padrões de memória $y^{p}, p=1, \ldots, m$ na $\mathrm{RNC}$ :

$$
x_{i}^{p} y_{i}^{p}>1, p=1, \ldots, m \quad e \quad i=1, \ldots, n .
$$

Substituindo-se a condição de existência de pontos de equilíbrio (6.2) na inequação acima, resulta: 


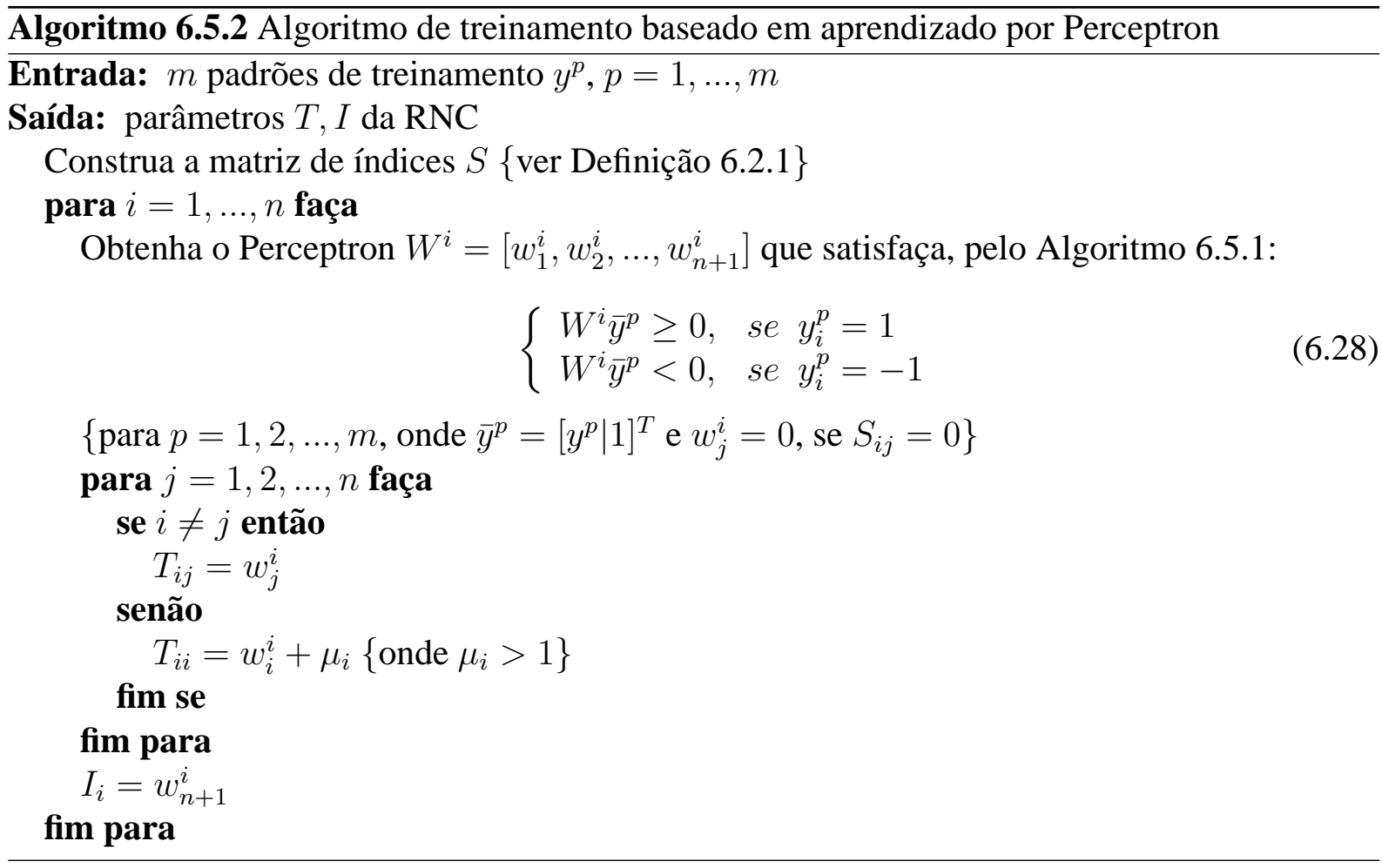

$$
y_{i}^{p}\left(T_{i} y^{p}+I_{i}\right)>1, \quad i=1, \ldots, n \quad e \quad p=1, \ldots, m \text {. }
$$

Também sabe-se que, pela equação (B.7) (Corolário B.3.2 no Apêndice B), se

$$
T_{i i} \geq 1, \quad i=1, \ldots, n
$$

os pontos de equilíbrio se encontrarão apenas na região de saturação $\left(\left|x_{i}^{p}\right| \geq 1, i=1, \ldots, n\right.$ e $p=1, \ldots, m)$. Baseado em (6.30) e (6.31), e utilizando as restrições de conectividade de RNCs dada pela matriz de índice $S$ (ver Seção 6.2.1), o Teorema 6.6.1 é formulado. Resultados desse teorema serão utilizados, mais adiante no texto, na síntese de memórias associativas em RNCs.

Teorema 6.6.1 Dada uma matriz de índice $S$ e um padrão $y^{*}$, suponha que, para $i=1, \ldots, n$, os parâmetros $T=T \mid S$ e I de (6.1) satisfaçam

$$
y_{i}^{*}\left(\sum_{j \in Z_{i}} T_{i j} y_{j}^{*}+I_{i}\right)>\kappa_{i} \max _{j \in Z_{i}}\left|T_{i j}\right|+\left(T_{i i}-1\right)
$$

onde $\kappa_{i} \geq 0$ e $Z_{i}=\left\{j \mid S_{i j}=1, j \neq i\right\}$. Então, qualquer vetor binário $y^{0} \in B^{n}$ tal que $y_{i}^{0} \neq y_{i}^{*}$ e que esteja a uma distância $\sum_{j \in Z_{i}}\left|y_{j}^{0}-y_{j}^{*}\right|<\kappa_{i}$ de $y^{*}$, tem as seguintes propriedades:

(a) Se $x(0)=y^{0}$ em (6.1), então $x_{i}(t)$ tende a $y_{i}^{*}$, em $t=0$; 
(b) o vetor $y^{0}$ não é armazenado no sistema (6.1)

Prova: ver Apêndice D.

Esse teorema atesta que existe uma bacia de atração, em torno de $y^{*}$, com tamanho $\kappa_{i}$ e que, portanto, qualquer padrão $y^{0}$, pertencente a esta bacia, convegirá para $y^{*}$.

Observação 6.6.2 A inequação (6.32) é equivalente a:

$$
y_{i}\left(\sum_{j \in Z_{i}} T_{i j} y_{j}+I_{i}\right)=T_{i i}+y_{i}\left(\sum_{j \in Z_{i}} T_{i j} y_{j}+I_{i}\right)>\kappa_{i} \max _{j \in}\left|T_{i j}\right|+\left(2 T_{i i}-1\right)
$$

que, com $\kappa_{i} \geq 0$ satisfazendo (6.32) e sob a condição de (6.31), implica

$$
y_{i}\left(\sum_{j \in Z_{i}} T_{i j} y_{j}+I_{i}\right)>1
$$

Portanto, o critério (6.32), proposto pelo Teorema 6.6.1, da mesma forma que (6.30), estabelece uma condição para que os padrões y $y^{p}$ sejam armazenados na rede.

O método proposto nessa seção explora a semelhança estrutural entre a formulação do problema, dada por (6.32), e LMIs (Linear Matrix Inequalities ou Inequações Matriciais Lineares), cuja definição é dada a seguir. Dessa forma, a regra de aprendizado que maximiza o tamanho das bacias de atração, em torno dos padrões de memória, consistirá da resolução de um GEVP (Generalized Eigenvalue Problem ou Problema de Autovalor Generalizado, ver Seção 6.6.1), caso este seja factível. Caso contrário, o problema se resume a um problema de otimização, formulado também como LMIs.

\subsubsection{LMIs e GEVPs}

Uma $L M I$ é uma restrição da forma:

$$
A(x)=A_{0}+x_{1} A_{1}+x_{2} A_{2}+\ldots+x_{n} A_{n}>0
$$

onde $x \in \mathbb{R}^{n}$ é uma variável e $A_{i}$ são matrizes simétricas constantes. O problema consiste em determinar $x$, tal que $A$ seja definida positiva ${ }^{1}$.

Um GEVP é uma generalização do problema de determinação de autovalores (e autovetores) de uma matriz $A$ que, no caso generalizado, envolve também uma matriz $B$ :

$$
A v=\lambda B v
$$

\footnotetext{
${ }^{1} A$ é definida positiva (denotada por $A>0$ ) se $x^{T} A x>0, \forall x \neq 0$.
} 
Neste caso, deseja-se conhecer quem são $\lambda$ e $v$ tal que o resultado da transformação linear operada por $A$ em $v$ seja um múltiplo escalar $\lambda$ da transformação linear de $B$ sobre esse mesmo vetor $v$. Entretanto, a solução do problema generalizado não é tão simples quanto a do problema tradicional $(A v=\lambda v)$. A solução pode ser encontrada considerando-se o seguinte problema de otimização, cujas restrições são LMIs:

$$
\begin{aligned}
& \min \\
& \text { s. a. }\left\{\begin{array}{l}
A x-\lambda B x>0 \\
B>0
\end{array}\right.
\end{aligned}
$$

O método apresentado utiliza a formulação dada por (6.33) para encontrar uma solução ao problema de construção de uma memória associativa em uma RNC.

\subsubsection{Estratégia de resolução utilizando LMIs}

Observe que em (6.32), conforme $\kappa_{i} \geq 0$ torna-se maior, as bacias de atração em torno dos padrões a serem armazenados se ampliam. Sendo esse efeito desejável, a estratégia de resolução a ser seguida é a de maximizar $\kappa_{i}$, quando (6.32) é factível. Maximizando os $\kappa_{i}$, por sua vez, implica em minimizar $T_{i i}$ em (6.32), que por (6.31) só podem assumir o valor mínimo de $T_{i i}=1$. Para $T_{i i}=1$ em (6.32), essa condição de factibilidade é equivalente a

$$
y_{i}\left(\sum_{j \in Z_{i}} T_{i j} y_{j}+I_{i}\right)>0, \quad i=1, \ldots, n
$$

Fixando-se $T_{i i}=1$, os parâmetros $T_{i j},\left(j \in Z_{i}\right)$ e $I_{i}$ podem ser encontrados maximizando $\kappa_{i} \geq 0$, tal que:

$$
y_{i}\left(\sum_{j \in Z_{i}} T_{i j} y_{j}+I_{i}\right)>\kappa_{i} \max _{j \in Z_{i}}\left|T_{i j}\right|, \quad i=1, \ldots, n
$$

Como esse problema de otimização tem restrições não-lineares, é impossível aplicar técnicas de programação linear para resolvê-lo. No entanto, o problema pode ser convertido em um GEVP, restringindo-se ao caso não-trivial em que $\max _{j \in Z_{i}}\left|T_{i j}\right| \neq 0$ e $\kappa_{i}>0$. Para isso, uma variável positiva e limitada $q_{i} \in(L, U), L>0$ e $U>0$ é introduzida em (6.35), que permite reescrever esse sistema de inequações como:

$$
y_{i}\left(\sum_{j \in Z_{i}} T_{i j} y_{j}+I_{i}\right)>\kappa_{i} q_{i}>\kappa_{i} \max _{j \in Z_{i}}\left|T_{i j}\right|, \quad i=1, \ldots, n
$$

As inequações anteriores podem ser separadas em 


$$
y_{i}\left(\sum_{j \in Z_{i}} T_{i j} y_{j}+I_{i}\right)-\kappa_{i} q_{i}>0, \quad i=1, \ldots, n
$$

$\mathrm{e}$

$$
\left|T_{i j}\right|<q_{i}, j \in Z_{i}
$$

Dessa forma, a estratégia de treinamento será: se (6.34) for factível, fixar $T_{i i}=1$ e encontrar $T_{i j},\left(j \in Z_{I}\right)$ e $I_{i}$ resolvendo

$$
\text { s.a. }\left\{\begin{array}{l}
\min \left(-\kappa_{i}\right) \\
\left(-\kappa_{i}\right) q_{i}+y_{i}\left(\sum_{j \in Z_{i}} T_{i j} y_{j}+I_{i}\right)>0, \quad i=1, \ldots, n \\
\left|T_{i j}\right|<q_{i}, j \in Z_{i} \\
\left|I_{i}\right|<U \\
L<q_{i}<U
\end{array}\right.
$$

Se a condição dada por (6.34) não for factível, a estratégia de solução proposta não é aplicável. Neste caso, apenas as restrições dadas por (6.30) e (6.31) devem ser utilizadas, e os parâmetros de interesse podem ser determinados a partir de:

$$
\left\{\begin{array}{l}
y_{i}\left(\sum_{j=1}^{n} T_{i j} y_{j}+I_{i}\right)=T_{i i}+y_{i}\left(\sum_{j \in Z_{i}} T_{i j} y_{j}+I_{i}\right)>1 \\
T_{i i} \geq 1, \quad i=1, \ldots, n
\end{array}\right.
$$

Quando $T_{i i}$ assume valores elevados, a condição anterior é trivialmente satisfeita. Entretanto, valores altos de $T_{i i}$ aumentam o número de estados espúrios da rede. Para eliminar esse efeito indesejável, fixa-se $T_{i i}=1+\epsilon$, onde $\epsilon$ é escolhido positivo e pequeno. Assim, quando (6.34) não é factível, faz-se: fixa-se $T_{i i}=1+\epsilon$ e encontram-se os parâmetros restantes, a partir da condição

$$
y_{i}\left(\sum_{j \in Z_{i}} T_{i j} y_{j}+I_{i}\right)>-\epsilon
$$

O Algoritmo 6.6.1 (ver Página 81) resume o procedimento de treinamento da rede utilizando LMIs. 


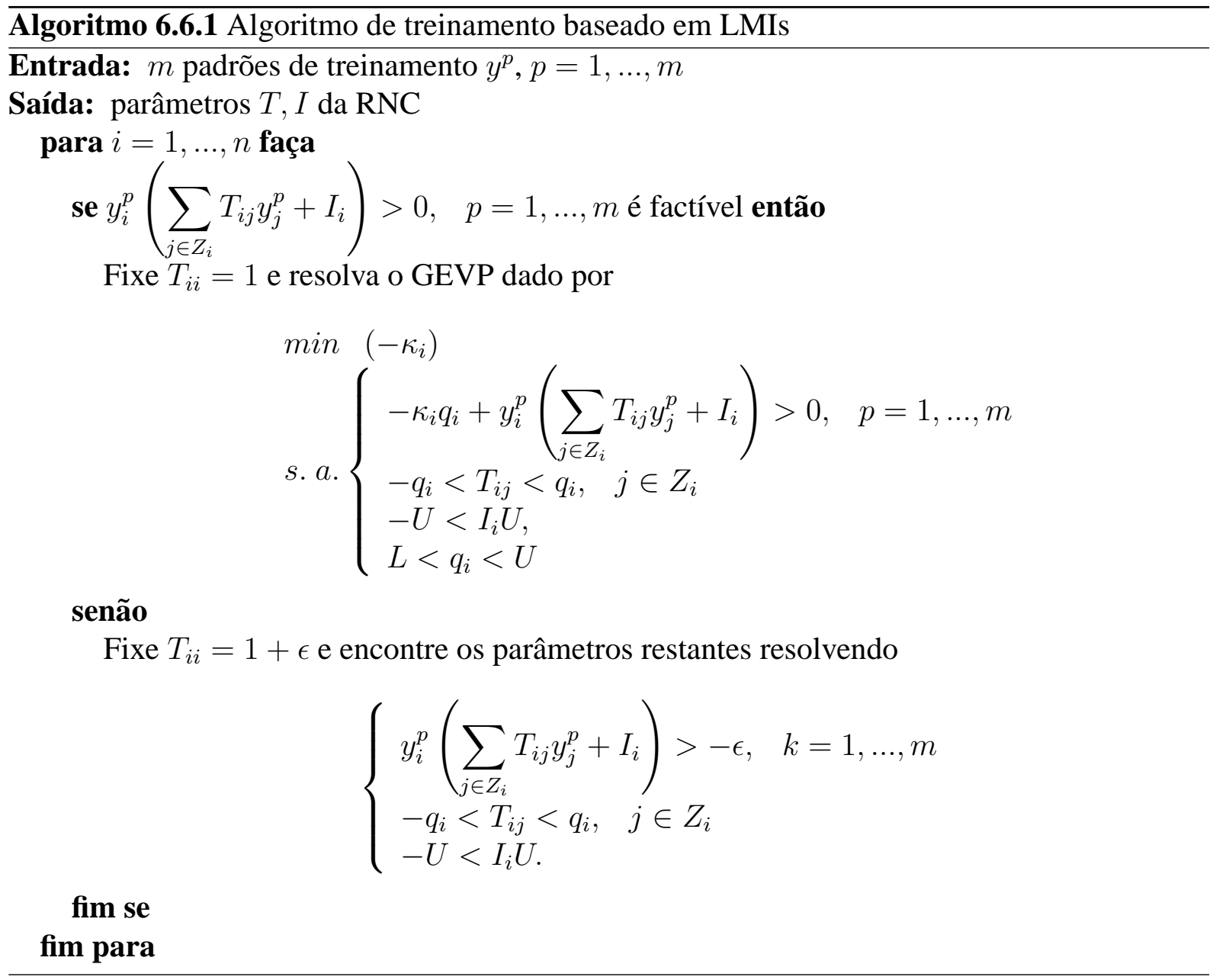

\subsection{Discussão}

Nesse capítulo, foram apresentados métodos de construção de memórias associativas em RNCs. Os métodos consistem de estratégias de resolução de um conjunto de equações (ou, alternativamente, de inequações), que descrevem tal problema.

Considerando-se a análise da estabilidade das RNCs treinadas pelos métodos apresentados nesse capítulo, cabe notar que tais métodos resultam em matrizes de conexões $T$ assimétricas. Isso impossibilita a análise de estabilidade utilizando-se funções de energia, como feito na Seção 4.2 e 5.2, pois não há um método sistemático para encontrar tais funções, nesse caso. Assim, só é possível obter resultados "locais" acerca da estabilidade dos pontos de equilíbrio conhecidos do sistema. Não se pode provar, portanto, que soluções periódicas ou caóticas não existirão na dinâmica resultante do sistema. Em [Liu \& Lu, 1997], por exemplo, é proposta uma modificação do algoritmo de treinamento baseado em Perceptrons, que leva em consideração essa preocupação quanto a estabilidade da rede. No artigo, é apresentado um algoritmo que resulta em uma matriz $T$ simétrica, garantindo, portanto, que apenas pontos de equilíbrios exis- 
tam na dinâmica da rede. Uma alternativa para a análise de redes com matrizes de conexões assimétricas, é discutida no Capítulo 8.

No capítulo seguinte, é apresentada uma simulação de todos os métodos apresentados nesse capítulo, comparando-os em um teste de desempenho, de forma que a capacidade de armazenamento e recuperação de padrões, para cada um dos métodos, é investigada. 


\section{Capítulo 7}

\section{Experimentos}

Com o objetivo de avaliar os métodos de construção de memórias associativas em RNCs, apresentados no Capítulo 6, testes comparativos de desempenho são apresentados neste capítulo. A Seção 7.1 descreve os experimentos realizados, enquanto a Seção 7.2 discute os resultados obtidos com os experimentos. Finalmente, a Seção 7.3 apresenta considerações finais a respeito dos testes.

\subsection{Características dos experimentos}

Os experimentos realizados foram implementados com auxílio do software MATLAB [The MathWorks, 2000]. Problemas de otimização, presentes no Algoritmo 6.6.1 da Seção 6.6.1, foram resolvidos por métodos de ponto-interior [Boyd et al., 1994], implementados pelas funções feasp e gevp do toolkit LMI, também do MATLAB. As RNCs consideradas, foram simuladas integrando-se numericamente as equações (6.1), por meio do algoritmo Runge-Kutta de quarta ordem [Press et al., 1993].

O desempenho das redes foi medido considerando-se o armazenamento e a recuperação de $m=26$ padrões compostos pelas letras de A-Z do alfabeto, como mostra a Figura 7.1. Cada subdivisão desta figura corresponde a um pixel da imagem, que é associado a uma célula da rede Portanto, as RNCs simuladas tiveram dimensões de $7 \times 7(n=49)$ células. Nas imagens consideradas, a cor branca corresponde ao valor -1 e a cor preta ao valor 1 , com valores intermediários representados em uma escala de tons de cinza.

Para testar a capacidade de recuperação de padrões armazenados, foram utilizados dois tipos de ruído: binário e gaussiano. O ruído binário consiste em inverter uma certa porcentagem de pixels da imagem. A Figura 7.2 mostra a recuperação do padrão "Y", corrompido com ruído binário (nesta imagem foram invertidos $6 \%$ dos pixels, o que correspondeu a 3 pixels). O ruído gaussiano, por sua vez, provoca uma perturbação nos padrões de acordo com uma distribuição 
normal de média e desvio padrão pré-estabelecidos. Na Figura 7.3, por exemplo, é ilustrada a recuperação do padrão "B" corrompido por ruído gaussiano, de desvio padrão $\sigma=1$ e média zero.

Para avaliar a capacidade de armazenamento da rede, a "taxa de carga" de padrões a que a RNC foi submetida variou de $10 \%$ a $50 \%$ do tamanho $n$ da rede. Essa taxa de carga indica a razão da quantidade de padrões armazenados, pela quantidade de células da rede. O parâmetro $r$ (raio da vizinhança das células, ver Seção 5.1) foi também variado em $r=1,2,3$, para se estudar o efeito do tamanho da vizinhança na capacidade de armazenamento.
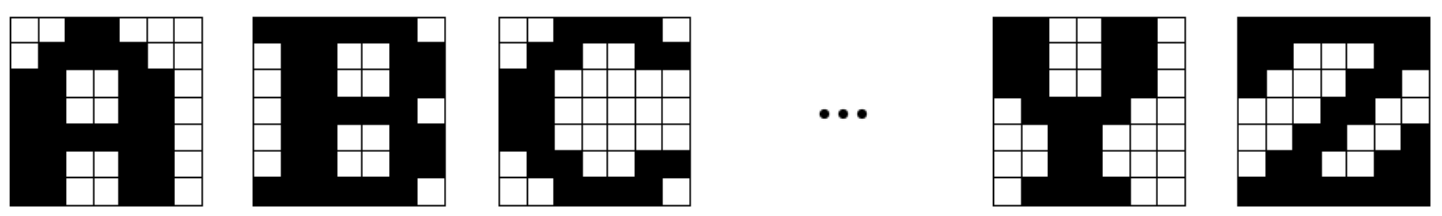

Figura 7.1: Padrões utilizados para o treinamento da rede.
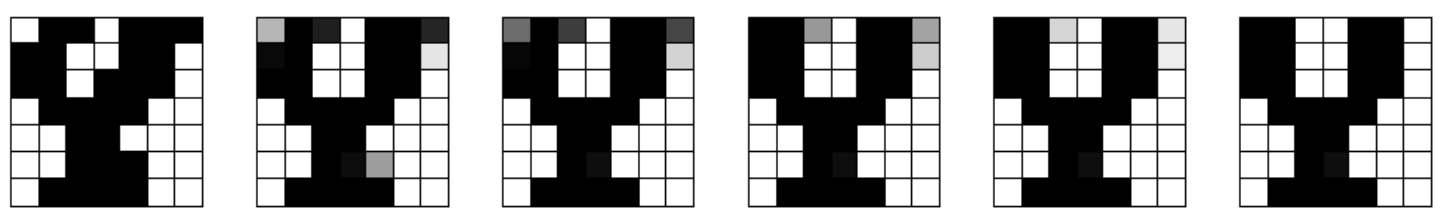

Figura 7.2: Exemplo de recuperação de padrão corrompido por ruído binário.
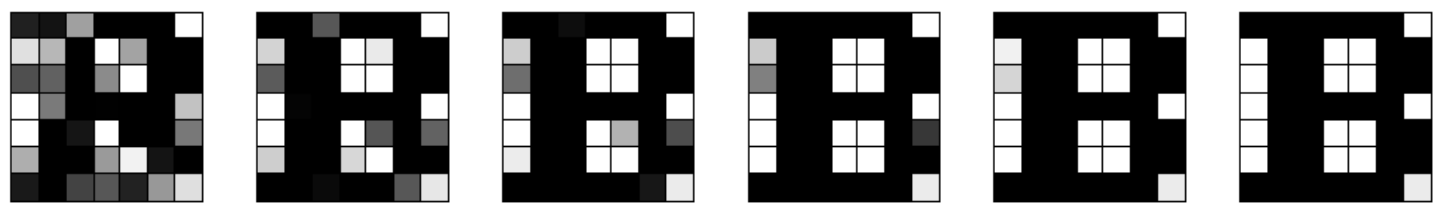

Figura 7.3: Exemplo de recuperação de padrão corrompido por ruído gaussiano.

No texto apresentado a seguir, considere a seguinte legenda: P-Inversa denota o método da Seção 6.2; Perceptron indica o método da Seção 6.5; SVD corresponde ao método da Seção 6.3; $H e b b$ denota o método da Seção 6.4 e $L M I$ corresponde ao método da Seção 6.6.

Parâmetros específicos de cada método foram também estudados quanto a influência no desempenho da rede. As variações estudadas para tais parâmetros estão abaixo relacionadas:

- P-Inversa: $\alpha \in\{2,3,5,7,10\}$; 
- Perceptron: $\mu \in\{1.2,2.0,3.0,5.0,7.0\}$;

- $S V D: \alpha \in\{2,3,5,7,10\}, w_{i}=\mu_{i} \cdot O_{m_{i}} \cdot U_{i 2}$, onde $\mu_{i} \in\{-10,-5,1,5,10\}$, $O=[1, \ldots, 1] \in R^{1 \times m_{i}}, m_{i}=\sum_{j=1}^{n} S_{i j}$ e $U_{i 2}$ como definido pela equação (C.2), no Apêndice $C$;

- Hebb: $\left(E_{L}, E_{R}\right) \in\{(1.1,3.1),(2.0,4.0),(4.0,6.0),(1.1,10.0),(1.1,21.1)\}$, $E=\left(E_{L}+E_{R}\right) / 2$

- LMI: $\epsilon \in\{0.0001,0.001,0.01,0.1,0.2\},(L, U) \in\{(1,3),(2,4),(4,6),(1,10),(1,20)\}$.

Para a comparação do desempenho dos diversos métodos, foi feita a seguinte escolha dos valores dos parâmetros que resultaram em um melhor desempenho de cada método:

- P-Inversa: $\alpha=7$;

- Perceptron: $\mu=1.2$;

- $S V D: \alpha=7$ e $\mu_{i}=1$;

- Hebb: $\left(E_{L}, E_{R}\right)=(1.1,3.1)$;

- LMI: $\epsilon=0.1,(L, U)=(1,10)$;

Os experimentos realizados utilizaram-se de dois conjuntos de padrões para medir o desempenho dos métodos: o conjunto de padrões de treinamento e o conjunto de padrões de teste. Foram gerados 10 conjuntos de padrões de treinamento para cada taxa de carga da RNC. Essa taxa foi variada em 10\%,20\%,30\%,40\% e $50 \%$ (que correspoderam ao armazenamento de $p=5,10,15,20,25$ padrões). Portanto, foram gerados $10 \times 5=50$ conjuntos de dados de treinamento para a rede. Cada conjunto de treinamento foi composto por 30 amostras de padrões escolhidos aleatoriamente dentre as 26 letras do alfabeto (ver Figura 7.1).

Os conjuntos de padrões de teste foram gerados a partir do conjuntos de padrões de treinamento, perturbando as amostras com ruído. Foram considerados 6 níveis de ruídos, tanto binário quanto gaussiano. Os níveis de ruído binário considerados foram de 2\%,4\%,6\%,10\%, 15\% e $20 \%$ de inversão dos pixels (ou bits) das imagens de letras do alfabeto. Os níveis de ruído gaussiano variaram em $0.3,0.5,0.7,1.0,1.5$ e 2.0 , onde os números indicam o desvio padrão da distribuição normal de média zero considerada. Cada conjunto de treinamento, com determinada taxa de carga da RNC, foi perturbado com os 2 tipos de ruído considerados, em 6 diferentes níveis. Foram gerados, portanto, $50 \times 2 \times 6=600$ conjuntos de exemplos de teste, cada um contendo 30 padrões corrompidos por ruídos.

O teste comparativo de desempenho consistiu de: 
1. Treinar as RNCs, utilizando cada um dos 5 métodos apresentados no Capítulo 6, com cada conjunto de padrões de treinamento construído;

2. Testar as redes treinadas, utilizando os conjuntos de padrões de teste gerados.

As redes treinadas utilizaram diferentes valores de $r$ (parâmetro de vizinhança da RNC) para verificar também a influência deste parâmetro na capacidade de armazenamento e recuperação.

\subsection{Resultados}

O sucesso na recuperação dos padrões foi contabilizado apenas quando $100 \%$ dos pixels foram corrigidos nas imagens consideradas. As tabelas que se encontram no Apêndice E, apresentam as taxas médias de recuperação e os desvios padrões obtidos com os experimentos. Os gráficos nas Figuras 7.4 e 7.5 resumem os principais resultados obtidos.

Para o gráfico da Figura 7.4, referente à vizinhança de raio $r=1$, tem-se que, em média, os métodos Hebb e LMI mantém-se ligeiramente superiores na recuperação de padrões, em comparação com os demais métodos. Essa tendência se confirma para vizinhanças de raio maior. Deve-se observar que em relação ao método $S V D$, que difere do método P-Inversa apenas pela adição do termo que compreende o vetor arbitrário $w_{i}$ (comparar as equações (6.12) e (6.19)), uma análise detalhada dos resultados mostrou que a adição desse termo degrada o desempenho do método $S V D$, ao invés de melhorá-lo.

Considerando-se os padrões corrompidos por ruído gaussiano e vizinhança $r=1$ (ver gráfico na Figura 7.5), o método LMI teve um desempenho inferior comparado ao teste com ruído binário. Uma análise mais detalhada, por meio de simulações, mostrou que os padrões recuperados pelo método diferiam muito pouco (em termos de norma Euclidiana) dos padrões que se objetivava buscar. Isso só aconteceu para um raio de vizinhança pequeno $(r=1)$, sendo que para valores mais altos do parâmetro $r$, o método mostrou uma taxa de recuperação mais adequada.

Um fator importante a ser investigado, que no entanto não foi medido nos experimentos, é o tempo de execução dos algoritmos testados. No entanto, pôde-se observa, por meio das simulações, que o tempo de execução de $L M I$ é muito maior que o tempo de execução dos outros métodos. Assim, uma análise mais detalhada da complexidade deste algoritmo é necessária. Em [Szolgay et al., 1997], é realizado uma comparação de métodos considerando-se o tempo de execução, onde o algoritmo implementado pelo método Hebb mostrou-se o mais eficiente.

Deve-se destacar também que o desempenho do método Perceptron foi insatisfatório, sendo necessário a introdução de uma modificação neste, para se conseguir os resultados reportados. A condição para o armazenamento dos padrões, estabelecida pelas inequações (6.28) do Algoritmo 6.5.2, foi modificada para a condição: 


$$
\left\{\begin{array}{l}
W^{i} \bar{y}^{p} \geq 1, \text { se } y_{i}^{p}=1, \quad i=1, \ldots, n \text { e } p=1, \ldots, m \\
W^{i} \bar{y}^{p}<1, \text { se } y_{i}^{p}=-1
\end{array}\right.
$$

Dessa forma, os pontos de equilíbrio são ajustados mais no interior da região de saturação, aumentando a robustez na recuperação, como discutido na Seção 6.1. 

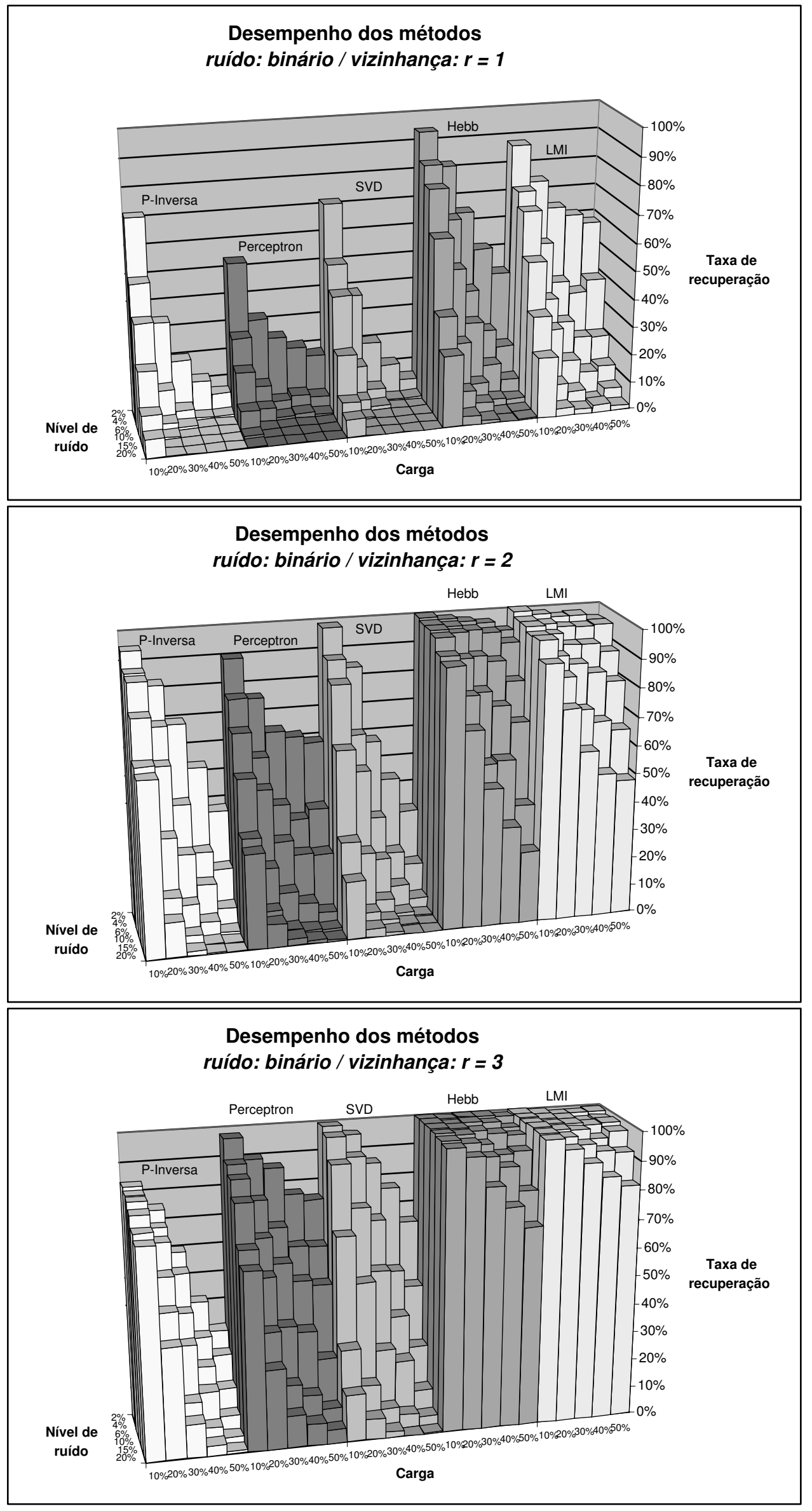

Figura 7.4: Gráficos de desempenho para ruído binário. 

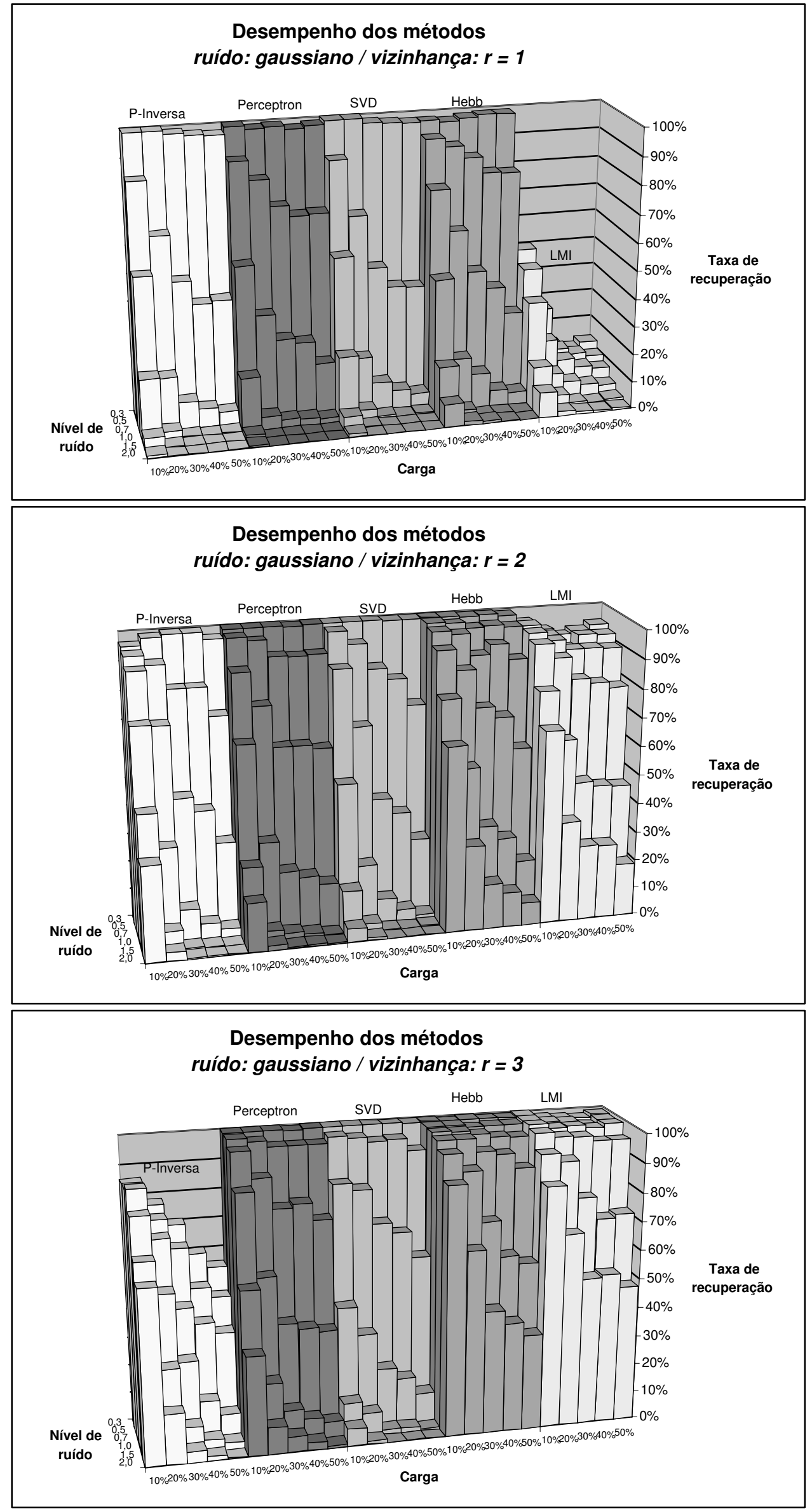

Figura 7.5: Gráficos de desempenho para ruído gaussiano. 


\subsection{Considerações Finais}

Para que fosse possível estabelecer uma comparação entre os diversos métodos testados, foi realizado um teste estatístico sobre os dados obtidos, denominado Teste de Hipóteses ou TesteT de Student [Morettin \& de O. Bussab, 2003]. Os resultados obtidos para um nível de significância de $5 \%$ são apresentados nas Tabelas 7.1 e 7.2 . Vê-se que, para quase a totalidade de testes envolvendo ruído binário, o desempenho dos métodos Hebb e $L M I$ foram sensivelmente melhores que os demais métodos apresentados. A análise feita com padrões corrompidos por ruído gaussiano, por sua vez, revelou que ambos os métodos estudados se mostraram aproximadamente equivalentes, de acordo com o teste estatístico realizado. Dessa forma, conclui-se a avaliação de desempenho dos métodos estudados.

Tabela 7.1: Melhores métodos - recuperação de padrões corrompidos por ruído binário.

\begin{tabular}{|r||c|c|c|}
\hline Carga & $\mathrm{r}=1$ & $\mathrm{r}=2$ & $\mathrm{r}=3$ \\
\hline \hline $10 \%$ & P-Inv, SVD, Hebb, LMI & Hebb, LMI & Hebb, LMI \\
\hline $20 \%$ & Hebb, LMI & Hebb, LMI & Hebb, LMI \\
\hline $30 \%$ & Hebb, LMI & Hebb, LMI & Hebb, LMI \\
\hline $40 \%$ & Percept, Hebb, LMI & Hebb, LMI & Hebb, LMI \\
\hline $50 \%$ & Hebb, LMI & Hebb, LMI & Hebb, LMI \\
\hline
\end{tabular}

Tabela 7.2: Melhores métodos - recuperação de padrões corrompidos por ruído gaussiano.

\begin{tabular}{|r||c|c|c|}
\hline Carga & $\mathrm{r}=1$ & $\mathrm{r}=2$ & $\mathrm{r}=3$ \\
\hline \hline $10 \%$ & Todos foram equivalentes & Todos foram equivalentes & Percept, Hebb, LMI \\
\hline $20 \%$ & Todos foram equivalentes & Todos foram equivalentes & Percept, Hebb, LMI \\
\hline $30 \%$ & Todos foram equivalentes & Todos foram equivalentes & Percept, Hebb, LMI \\
\hline $40 \%$ & Todos foram equivalentes & Todos foram equivalentes & Percept, Hebb, LMI \\
\hline $50 \%$ & Todos foram equivalentes & Todos foram equivalentes & Percept, Hebb, LMI \\
\hline
\end{tabular}




\section{Capítulo 8}

\section{Conclusões}

O presente estudo consistiu da investigação de modelos de processamento de informação inspirados no cérebro. Mais especificamente, decidiu-se investigar modelos de memória associativa implementadas por redes neurais realimentadas. Sendo essas redes modeladas como sistemas não-lineares de alta dimensionalidade, foi necessário o estudo de conceitos fundamentais de sistemas dinâmicos para o desenvolvimento da pesquisa.

A abordagem utilizada para se concretizar os objetivos propostos, baseou-se na investigação de dois modelos de RNAs: o modelo clássico de Hopfield e o modelo de RNCs. Como as RNCs representam uma extensão do modelo de Hopfield, estas foram utilizadas para se aprofundar as análises desenvolvidas. Foram realizadas análises de estabilidade das redes, assim como análises experimentais, nesse caso envolvendo apenas o modelo de RNCs. A análise experimental consistiu da realização de testes que avaliaram o desempenho de métodos de construção de memórias associativas em RNCs.

Como resultado do trabalho desenvolvido, em termos de formação e conhecimentos adquiridos, obteve-se:

- Introdução e familiarização ao uso de ferramentas de análise de sistemas não-lineares;

- Exploração de tópicos de álgebra linear, em uma abordagem aplicada;

- Familiarização com métodos numéricos e de simulação de sistemas de EDOs, entre outros.

Esses resultados são relevantes para a formação do pesquisador, pois a capacidade de lidar com modelos matemáticos e computacionais são essenciais para a investigação de fenômenos complexos, como é o caso do funcionamento do cérebro.

Com relação à análise de sistemas não-lineares, um resultado considerado importante foi obtido quanto à investigação teórica da origem da existência da condição de simetria (ver equações 
(4.4) e 5.3), Capítulos 4 e 5, respectivamente) sobre a matriz de conexões do modelo de Hopfield e das RNCs. Tal simetria, como indicou a Proposição 2.5.11 formulada, é uma condição necessária para a existência de uma função de energia, utilizada para provar a estabilidade das referidas redes. Este resultado foi importante porque na literatura de redes neurais tal condição não é explicada, apenas citada.

A presente pesquisa também propiciou resultados acerca da comparação do desempenho de métodos de construção de memórias associativas em RNCs. A abordagem utilizada foi criteriosa e baseada em condições semelhantes de avaliação dos diversos métodos propostos, sendo que tal abordagem era inexistente na literatura. Os resultados obtidos com os testes de desempenho se mostraram relevantes por proporcionarem condições de escolha da melhor estratégia de treinamento de uma RNC, em aplicações envolvendo memórias associativas. Um dos resultados mais importantes desse estudo talvez seja quanto à viabilidade de aplicação de redes neurais realimentadas em problemas envolvendo o Reconhecimento de Padrões e o Processamento de Imagens. Especialmente se tais redes forem implementadas em hardware, os resultados poderão ser muito promissores.

Como indicaram as tabelas de resultados dos testes de desempenho realizados (ver Apêndice E), as taxas de recuperação de padrões obtidas foram altas, podendo vir a serem comparadas, em um trabalho futuro, a outras estratégias de reconhecimento de padrões existentes. Os resultados obtidos com os testes podem ser também utilizados para se guiar a pesquisa de novos métodos de aprendizado, além da melhoria dos métodos existentes. As melhores estratégias que se descobriu para RNCs, poderiam ser adaptadas para serem utilizadas no treinamento de outros modelos de redes neurais realimentadas existentes, ou a se desenvolver.

A seguir, foram relacionadas algumas possíveis direções futuras para a presente pesquisa:

- Mostrar a viabilidade de utilização de RNCs em Reconhecimento de Padrões e/ou Processamento de Imagens, por meio da implementação de uma aplicação real. Poder-se-ia desenvolver, por exemplo, um módulo de visão computacional robótico.

- Analisar a estabilidade de RNCs com matrizes assimétricas, como as obtidas com os métodos de treinamento apresentados no Capítulo 6. Em [Khalil, 1996], por exemplo, é apresentada uma análise do modelo de Hopfield com matrizes desse tipo, considerando-o como se formado por muitos subsistemas interconectados. Sob algumas hipóteses relativas à magnitude dos parâmetros, pode-se obter resultados teóricos quanto à estabilidade do sistema, removendo-se a restrição de simetria sob a matriz de conexões. Tal análise poderia, então, ser estendida para o caso de RNCs;

- Investigar as bases biológicas do processamento de informação, plasticidade sináptica e/ou formação de memória no cérebro, visando o desenvolvimento de modelos computacionais mais eficientes e/ou mais biologicamente plausíveis. 
Apesar das dificuldades de análise e de aplicação de redes neurais realimentadas em problemas reais, o presente estudo revelou que essas tarefas são possíveis de serem realizadas. Com as inovações tecnológicas em integração de circuitos, em breve deve-se tornar possível implementar grandes redes dinâmicas diretamente em hardware, o que representará avanços tanto no desenvolvimento de aplicações, como na investigação de modelos biologicamente plausíveis do cérebro. $\mathrm{O}$ estudo dos modelos matemáticos e computacionais, realizados durante essa pesquisa, proporcionaram ao pesquisador a consolidação de uma razoável base matemática que, no futuro, pode vir a ser utilizada na investigação e, quem sabe, na elucidação dos mistérios envolvidos no processamento de informação no cérebro. 


\section{Referências Bibliográficas}

[Boyd et al., 1994] Boyd, S., ElGhaoui, L., Feron, E., \& Balakrishnan, V. (1994). Linear matrix inequalities in systems and control theory. SIAM, Philadelphia, PA.

[Carpenter \& Grossberg, 1995] Carpenter, G. \& Grossberg, S. (1995). Adaptive resonance theory (art). In Arbib, M., editor, Handbook of brain theory and neural networks, pages 79-82. MIT Press.

[Chua \& Yang, 1988] Chua, L. O. \& Yang, L. (1988). Cellular neural networks: theory. IEEE Transactions on Systems and Circuits, 35(10):1257-1272.

[Coolen, 1998] Coolen, A. C. C. (1998). Concepts for neural networks - a survey, chapter A beginner's guide to the mathematics of neural networks, pages 13-70. Springer.

[Crounse \& Chua, 1997] Crounse, K. R. \& Chua, L. O. (1997). A new synthesis approach for feedback neural networks based on the perceptron training algorithm. IEEE Transactions on Circuits and Systems, 42(10):583-601.

[Devaney, 1989] Devaney, R. L. (1989). An introduction to chaotic dynamical systems. Addison-Wesley, Redwood City, California.

[Elaydi, 1999] Elaydi, S. (1999). An introduction to difference equations. Springer, New York.

[Grassi, 2001] Grassi, G. (2001). On discrete-time cellular neural networks for associative memories. IEEE Transactions on Circuits and Systems-I: Fundamental Theory and Applications, 48(1):107-111.

[Guidorizzi, 2002] Guidorizzi, H. L. (2002). Um curso de cálculo, volume 3. LTC, Rio de Janeiro, 5 edition.

[Hartman, 1964] Hartman, P. (1964). Ordinary Differential Equations. Wiley, New York.

[Hebb, 1949] Hebb, D. O. (1949). The organization of behavior. Wiley, New York. 
[Hodgkin \& Huxley, 1952] Hodgkin, A. L. \& Huxley, A. F. (1952). A quantitative description of ion currents and its applications to conduction and excitation in nerve membranes. Journal of Physiology, 117:500-544.

[Hopfield, 1982] Hopfield, J. J. (1982). Neural networks and physical systems with emergent collective computational abilities. Proceedings of the National Academy of Sciences of the USA, 79:2554-2558.

[Hopfield \& Tank, 1985] Hopfield, J. J. \& Tank, D. W. (1985). "neural” computation of decisions in optimization problems. Biological Cybernetics, 52:141-152.

[Ising, 1924] Ising, E. (1924). Beitrag zur Theorie des Ferro- und Paramagnetismus. PhD thesis, Universidade de Hamburgo, Hamburgo.

[Jr. \& de Melo, 1982] Jr., J. P. \& de Melo, W. (1982). Geometric theory of dynamical systems : an introduction. Springer-Verlag, New York.

[Kaneko, 1993] Kaneko, K. (1993). Theory and applications of coupled map lattices. John Wiley, England.

[Khalil, 1996] Khalil, H. (1996). Nonlinear systems. Prentice-Hall.

[Kohonen, 1997] Kohonen, T. (1997). Self-organising maps. Springer-Verlag, 2nd edition.

[Ladeira, 2001] Ladeira, L. A. C. (2001). Teoria qualitativa de equações diferenciais. Technical report, ICMC-USP.

[Lay, 1996] Lay, D. C. (1996). Linear algebra and its applications. Addison-Wesley, 2nd edition.

[Li et al., 1989] Li, J.-H., Michel, A. N., \& Porod, W. (1989). Analysis and synthesis of a class of neural networks: linear systems operating on a closed hypercube. IEEE Transactions on Circuits and Systems, 36(11):1405-1422.

[Lim, 1990] Lim, J. S. (1990). Two-dimensional signal and image processing. Prentice Hall, Englewood Cliffs, NJ.

[Little, 1974] Little, W. A. (1974). The existence of persistent states in the brain. Mathematical Biosciences, (19):101-120.

[Liu \& Lu, 1997] Liu, D. \& Lu, Z. (1997). A new synthesis approach for feedback neural networks based on the perceptron training algorithm. IEEE Transactions on Neural Networks, 8(6):1468-1482. 
[Liu \& Michel, 1994] Liu, D. \& Michel, A. N. (1994). Sparsely interconnected neural networks for associative memories with applications to cellular neural networks. IEEE Transactions on Circuits and Systems II, 41:295-307.

[McCulloch \& Pitts, 1943] McCulloch, W. S. \& Pitts, W. (1943). A logical calculus of the ideas immanent in nervous activity. Bulletin of Mathematical Biophysics, 5:115-133.

[Minsky \& A., 1969] Minsky, M. L. \& A., P. S. (1969). Perceptrons. MIT Press, Cambridge.

[Mirollo \& Strogatz, 1990] Mirollo, R. \& Strogatz, S. (1990). Synchronization of pulsecoupled biological oscillators. SIAM J. Appl. Math., 50:1645-1662.

[Morettin \& de O. Bussab, 2003] Morettin, P. A. \& de O. Bussab, W. (2003). Estatística básica. Saraiva, São Paulo, 5 edition.

[Ott, 1993] Ott, E. (1993). Chaos in dinamical systems. Cambridge University Press, New York.

[Park et al., 2001] Park, J., Kim, H. Y., \& Lee, S. W. (2001). A synthesis procedure for associative memories based on space-varying cellular neural networks. Neural Networks, (14):107113.

[Personnaz et al., 1986] Personnaz, L., Guyon, I., \& Dreyfus, G. (1986). Collective computational properties of neural networks: new learning mechanisms. Phys. Rev. A, 34(5):42174228.

[Press et al., 1993] Press, W. H., Flannery, B. P., Teukolsky, S. A., \& Vetterling, W. T. (1993). Numerical recipes in $C$ : the art of scientific computing. Cambridge University Press.

[Rosenblatt, 1958] Rosenblatt, F. (1958). The perceptron: probabilistic model for information storage and organization in the brain. Psychological Review, (65):386-408.

[Rosenblatt, 1962] Rosenblatt, F. (1962). Principles of neural dynamics. Spartan, New York.

[Rosenfeld \& Kak, 1982] Rosenfeld, A. \& Kak, A. C. (1982). Digital picture processing. Academic, New York.

[Rumelhart \& McClelland, 1986] Rumelhart, D. E. \& McClelland, K. L. (1986). Parallel distributed processing, volume 1. MIT Press, Cambridge, MA.

[Skarda \& Freeman, 1982] Skarda, C. A. \& Freeman, W. J. (1982). How brains make chaos in order to make sense of the world. Brain Behavioral Sci., 10:161-195. 
[Strogatz, 1989] Strogatz, S. H. (1989). Nonlinear dynamics and chaos: with applications to physics, biology, chemistry, and engineering. Addison-Wesley, Reading, Massachusets.

[Szolgay et al., 1997] Szolgay, P., Szatmári, I., \& László, K. (1997). A fast fixed point learning method to implement associative memory on cnns. IEEE Transactions on Circuits and Systems-I: Fundamental Theory and Applications, 44(4):362-366.

[The MathWorks, 2000] The MathWorks, I. (2000). Matlab. CD-ROM. Versão 6, Release 12.

[Werblin et al., 1995] Werblin, F., Roska, T., \& Chua, L. (1995). The analogic cellular neural network as a bionic eye. Int. J. Circuit Theory and Applications, 23:541-569.

[Werbos, 1974] Werbos, P. (1974). Beyond regression: new tools for prediction and analysis in behavioral sciences. $\mathrm{PhD}$ thesis, Harvard University.

[Widrow \& M.E., 1960] Widrow, B. \& M.E., H. (1960). Adaptive switching circuits. In IRE WESCON Convention Record, volume 4, pages 96-104, New York. IRE. 


\section{Apêndice A}

\section{Forma Canônica de Jordan}

Lema A.0.1 (Forma Canônica de Jordan) Seja A uma matriz $n \times n$, cujos autovalores distintos são

$$
\lambda_{1}, \ldots, \lambda_{p}, \lambda_{p+1}, \ldots, \lambda_{p+m}
$$

Então, existe uma matriz invertível $M$ tal que $J=M^{-1} A M$ é da forma

$$
J=\left[\begin{array}{llll}
J_{0} & 0 & \ldots & 0 \\
0 & J_{1} & \ldots & 0 \\
\vdots & \vdots & \ddots & \vdots \\
0 & 0 & \ldots & J_{m}
\end{array}\right]
$$

onde $J_{0}, J_{1}, \ldots, J_{m}$ são matrizes quadradas (chamadas blocos de Jordan), de ordens $p, p_{1}, \ldots, p_{m}, \operatorname{com} p+p_{1}+\cdots+p_{m}=n, J_{0}=\operatorname{diag}\left(\lambda_{1}, \ldots, \lambda_{p}\right)$ e, paracada $k=1, \ldots, m$

$$
J_{k}=\left[\begin{array}{llllll}
\lambda_{p+k} & 1 & 0 & \ldots & 0 & 0 \\
0 & \lambda_{p+k} & 1 & \ldots & 0 & 0 \\
0 & 0 & \lambda_{p+k} & \ldots & 0 & 0 \\
\vdots & \vdots & \vdots & \ddots & \vdots & \vdots \\
0 & 0 & 0 & \ldots & \lambda_{p+k} & 1 \\
0 & 0 & 0 & \ldots & 1 & \lambda_{p+k}
\end{array}\right]
$$

Observação A.0.2 Quando A é diagonalizável no Lema A.0.1, a matriz P é aquela cujas colunas são os autovetores de A. Quando A não é diagonalizável, P éformada pelos autovetores generalizados de $A$. 


\section{Apêndice B}

\section{Análise do modelo de RNCs}

\section{B.1 Introdução}

Considere o seguinte sistema de equações diferencias, na análise que segue

$$
\left\{\begin{array}{l}
\dot{x}(t)=-A x(t)+T y(x)+I \\
y(x)=\operatorname{sat}(x)
\end{array}\right.
$$

onde $x: \mathbb{R} \rightarrow \mathbb{R}^{n}, y: \mathbb{R}^{n} \rightarrow D^{n}\left(D^{n}:=\left\{x \in \mathbb{R}^{n}:-1 \leq x_{i} \leq 1, i=1, \ldots, n\right\}\right), A=$ $\operatorname{diag}\left(a_{1}, \ldots, a_{n}\right) \in \mathbb{R}^{n \times n}, \operatorname{com} a_{i}>0$ para $i=1, \ldots, n, T=\left[T_{i j}\right] \in \mathbb{R}^{n \times n}$ é uma matriz com coeficientes constantes, $I \in \mathbb{R}^{\ltimes}$ é um vetor constante, $\dot{x}=\frac{d x}{d t}$ e $\operatorname{sat}(x)=\left[\operatorname{sat}\left(x_{1}\right) \cdots \operatorname{sat}\left(x_{n}\right)\right]^{T}$, onde

$$
\operatorname{sat}\left(x_{i}\right)= \begin{cases}-1, & \text { se } x_{i}<-1 \\ x_{i}, & \text { se }-1 \leq x_{i} \leq 1 \\ 1, & \text { se } x_{i}>1\end{cases}
$$

Se $A=\operatorname{diag}(1, \ldots, 1)$, então a equação (B.1) descreve o modelo de Redes Neurais Celulares (Capítulo 5) dado pela equação (5.4).

\section{B.2 Notação}

Para $m$ inteiro, $0 \leq m \leq n$, seja

$\Lambda_{m}=\left\{\xi=\left[\xi_{1}, \ldots, \xi_{n}\right]^{T} \in \Lambda: \xi_{\sigma_{i}}=0,1 \leq i \leq m\right.$, e $\xi_{\sigma_{i}}= \pm 1, m<i \leq n$, para algum $\left.\sigma \in P(n)\right\}$

onde $\Lambda=\left\{\xi=\left[\xi_{1}, \ldots, \xi_{n}\right]^{T}: \xi_{i}= \pm 1\right.$ ou $\xi_{i}=0$, para $\left.1 \leq i \leq n\right\}$ e $P(n)$ denota o conjunto de 
todas as permutações em $\{1, \ldots, n\}$.

Para $\xi \in \Lambda$, seja

$C(\xi)=\left\{x=\left[x_{1}, \ldots, x_{n}\right]^{T} \in \mathbb{R}^{n}:\left|x_{i}\right|<1\right.$, se $\xi_{i}=0 ; x_{i} \geq 1$, se $\xi_{i}=1 ; x_{i} \leq-1$, se $\left.\xi_{i}=-1\right\}$

Suponha $\xi \in \Lambda_{m}$ e $\sigma \in P(n)$ tal que

$$
\xi_{\sigma_{i}}=0, \text { para } 1 \leq i \leq m \mathrm{e} \xi_{\sigma_{i}}= \pm 1, \text { para } m<i \leq n
$$

Denote

$$
\begin{aligned}
A_{I} & =\operatorname{diag}\left[a_{\sigma_{1}}, \ldots, a_{\sigma_{m}}\right], \\
A_{I I} & =\operatorname{diag}\left[a_{\sigma_{m+1}}, \ldots, a_{\sigma_{n}}\right], \\
T_{I, I} & =\left[T_{\sigma_{i} \sigma_{j}}\right]_{1 \leq i, j \leq m}, \\
T_{I, I I} & =\left[T_{\sigma_{i} \sigma_{j}}\right]_{1 \leq i \leq m, m \leq j \leq n}, \\
T_{I I, I} & =\left[T_{\sigma_{i} \sigma_{j}}\right]_{m \leq i \leq n, 1 \leq j \leq m}, \\
T_{I I, I I} & =\left[T_{\sigma_{i} \sigma_{j}}\right]_{m \leq i, j \leq n}, \\
I_{I} & =\left[I_{\sigma_{1}}, \ldots, I_{\sigma_{m}}\right]^{T}, \\
I_{I I} & =\left[I_{\sigma_{m+1}}, \ldots, I_{\sigma_{n}}\right]^{T}, \\
\xi_{I} & =\left[\xi_{\sigma_{1}}, \ldots, \xi_{\sigma_{m}}\right]^{T} \\
\xi_{I I} & =\left[\xi_{\sigma_{m+1}}, \ldots, \xi_{\sigma_{n}}\right]^{T} .
\end{aligned}
$$

Observação B.2.1 Para um dado $\xi \in \Lambda_{m}$, podem existir diferentes elementos em $P(n)$ tal que (B.2) é verdadeira. Para cada diferentes permutação, a notação dada acima é a mesma, independente da ordenação utilizada nos componentes de $\xi$.

Observação B.2.2 Se $m=n$, tem-se que $A_{I}=A, T_{I, I}=T, I_{I}=I, \xi_{I}=\xi$, e não existem $A_{I I}, T_{I, I I}, T_{I I, I}, T_{I I, I I}, I_{I I}, \xi_{I I}$. Se $m=0$, tem-se que $A_{I I}=A, T_{I I, I I}=T, I_{I I}=I, \xi_{I I}=\xi$, e não existem $A_{I}, T_{I, I}, T_{I, I I}, T_{I I, I}, I_{I}, \xi_{I}$.

\section{B.3 Resultados}

Considere $\xi \in \Lambda_{m}, 0<m<n$, com $\sigma \in P(n)$ tal que $\xi_{\sigma_{i}}=0,1 \leq i \leq m$ e $\xi_{\sigma_{i}}=$ $\pm 1, m<i \leq n$. Pode-se escrever a equação (B.1) como

$$
\left\{\begin{array}{l}
\dot{x}_{I}=-A_{I} x_{I}+T_{I, I} x_{I}+T_{I, I I} \xi_{I I}+I_{I} \\
\dot{x}_{I I}=-A_{I I} x_{I} I+T_{I I, I} x_{I}+T_{I I, I I} \xi_{I I}+I_{I I}
\end{array}\right.
$$


onde $\xi_{I I}=\left[\xi_{\sigma_{m+1}}, \ldots, \xi_{\sigma_{n}}\right]^{T}, x_{I}=\left[x_{\sigma_{1}}, \ldots, x_{\sigma_{m}}\right]^{T}$ com $-1<x_{\sigma_{i}}<1$ para $1 \leq i \leq m$, e $x_{I I}=\left[x_{\sigma_{m+1}}, \ldots, x_{\sigma_{n}}\right]^{T}, \operatorname{com} \xi_{\sigma_{i}} x_{\sigma_{i}} \geq 1$ para $m<i \leq n$.

A equação (B.4) é dita uma representação linear equivalente de (B.1) sobre a região $C(\xi)$ pois, com a notação utilizada, é possível separar as variáveis de (B.1) que operam na região linear $\left(\left|x_{i}\right|<1, i=1, \ldots, n\right)$, denotadas $x_{I}(t)$, das que operam na chamada região de saturação $(|x i| \geq 1, i=1, \ldots, n)$, denotadas $x_{I I}(t)$.

Também, devido à notação utilizada:

- Quando $m=n$, tem-se que $\Lambda_{n}=0$. Nesse caso, para $x \in C(0)=\left(D^{n}\right)^{0}$ (onde $\left(D^{n}\right)^{0}$ denota o interior do conjunto $D^{n}$ ), o sistema (B.1) fica

$$
\dot{x}=(T-A) x+I .
$$

- Quando $m=0$, tem-se $\Lambda_{0}=B^{n}$. Nesse caso, para $x \in \Lambda_{0}, x \in C(\xi)$, o sistema (B.1) fica

$$
\dot{x}=-A x+T \xi+I .
$$

Considere, agora, a seguinte hipótese para o sistema (B.1):

(H1) Para algum $m, 0<m \leq n$, e para algum $\xi \in \Lambda_{m}$, a matriz

$$
T_{I, I}-A_{I}=\left[T_{\sigma(i) \sigma(j)}\right]_{1 \leq i, j \leq m}-\operatorname{diag}\left[a_{\sigma(i)}, \ldots, a_{\sigma(m)}\right]
$$

é não singular, onde $\sigma \in P(n)$ tal que $\xi_{\sigma(i)}=0$, para $1 \leq i \leq m$ e $\xi_{\sigma(i)} \pm 1$, para $m<i \leq n$.

Para $x_{\xi}$ um ponto de equilíbrio de (B.1), suponha que a Hipótese (H1) é satisfeita. O equilíbrio $x_{\xi}$ é tal que:

(a) Se $\xi=0 \in \Lambda_{n}$ (isto é, se $m=n$ ), tem-se que

$$
x_{\xi}=(A-T)^{-1} I
$$

(b) Se $\xi \in \Lambda_{m}$, para $0<m<n$, tem-se que

$$
x_{\xi}=\left[x_{\xi}(1), \ldots, x_{\xi}(n)\right]^{T} \in \mathbb{R}^{n}
$$


onde

$$
\begin{aligned}
x_{\xi I} & =\left[x_{\xi}(\sigma(1)), \ldots, x_{\xi}(\sigma(m))\right]^{T} \\
& =\left(A_{I}-T_{I, I}\right)^{-1}\left(T_{I, I I} \xi_{I I}+I_{I}\right),
\end{aligned}
$$

e

$$
\begin{aligned}
x_{\xi I I} & =\left[x_{\xi}(\sigma(m+1)), \ldots, x_{\xi}(\sigma(n))\right]^{T} \\
& =A_{I I}^{-1}\left(T_{I I, I} x_{\xi I}+T_{I I, I I} \xi_{I I}+I_{I I}\right) .
\end{aligned}
$$

para $A_{I}, A_{I I}, T_{I, I}, T I, I I, T_{I I, I}, T I I, I I, \xi_{I I}, I I$ e $I I I$ como definidos acima no texto;

(c) $\operatorname{Se} \xi \in \Lambda_{0}=B^{n}$ (isto é, se $m=0$ ), tem-se que

$$
x_{\xi}=A^{-1}(T \xi+I)
$$

Considere, também, a seguinte hipótese, nos resultados que seguem:

(H2) Seja (B.1) satisfazendo a Hipótese (H1). Para algum $\xi \in \Lambda_{m}, 0 \leq m \leq n$, assuma que um ponto de equilíbrio $x_{\xi}$ de (B.1) satisfaça $x_{\xi} \notin \partial(C(\xi))$ (onde $\partial(C(\xi))$ denota a fronteira do conjunto $C(\xi)$, isto é, $\left.x_{\xi} \neq \pm 1\right)$.

A partir dessas definições, pode-se formular o teorema que segue.

Teorema B.3.1 Suponha que (B.1) satisfaça as Hipóteses (H1) e (H2) acima. Para algum $m, 0 \leq m \leq n$ e para algum $\xi \in \Lambda_{m}$, tem-se que:

Caso I: se $m=n, \xi=0 \in \Lambda_{n}\left(C(\xi)=\left(D^{n}\right)^{0}\right)$ e, além disso,

(i) se $x_{\xi} \notin\left(D^{n}\right)^{0}$, então não existe nenhum equilíbrio de (B.1) em $\left(D^{n}\right)^{0}$.

(ii) se $x_{\xi} \in\left(D^{n}\right)^{0}$, então $x_{\xi}$ é o único equilíbrio de $(B .1)$ em $\left(D^{n}\right)^{0}$. Neste caso,

(a) se T - A tem um ou mais autovalores com parte real positiva, $x_{\xi}$ é instável;

(b) se todos autovalores de $T-A$ tem parte real negativa, $x_{\xi}$ é assintoticamente estável.

Caso II: se $0<m<n, \xi \in \Lambda_{m}$ e, além disso,

(i) se $x_{\xi} \notin C(\xi)$, então não existe nenhum equilíbrio de (B.1) em $C(\xi)$.

(ii) se $x_{\xi} \in C(\xi)$, então $x_{\xi}$ é o único equilíbrio de (B.1) em $C(\xi)$. Neste caso,

(a) se $T_{I, I}-A_{I}$ tem um ou mais autovalores com parte real positiva, $x_{\xi}$ é instável;

(b) se todos autovalores de $T_{I, I}-A_{I}$ tem parte real negativa, $x_{\xi}$ é assintoticamente estável. 
Caso II: se $m=0, \xi \in \Lambda_{0}=B^{n}$ e, além disso,

(a) se $x_{\xi} \notin C(\xi)$, então não existe nenhum equilíbrio de (B.1) em $C(\xi)$.

(b) se $x_{\xi} \in C(\xi)$, então $x_{\xi}$ é um equilíbrio assintoticamente estável de (B.1).

Prova: Para cada $\xi \in \Lambda_{m}, 0 \leq m \leq n$, considere (B.4)-(B.6). Os resultados do teorema decorrem diretamente da teoria de equações diferenciais lineares.

Os seguintes resultados seguem do Teorema B.3.1.

Corolário B.3.2 Suponha $T=\left[T_{i j}\right]$ simétrica em (B.1) e que os coeficientes $A=$ $\operatorname{diag}\left(a_{1}, \ldots, a_{n}\right)$ de (B.1) satisfaçam

$$
T_{i i}>a_{i}, \quad \text { para } i=1, \ldots, n .
$$

Então, todo ponto de equilíbrio assintoticamente estável $x_{e}=\left[x_{e 1}, \ldots, x_{e n}\right]^{T}$ de (B.1) satisfaz a condição

$$
\left|x_{e i}\right|>1, \quad \text { para } i=1, \ldots, n \text {. }
$$

Prova: Se (B.7) é satisfeita, as matrizes $T_{I, I}-A I$ e $T-A$ terão um ou mais autovalores não-negativos, já que são matrizes simétricas com elementos diagonais positivos. Portanto, os casos I e II do Teorema B.3.1 nunca podem ser satisfeitos, sendo que os pontos de equilíbrios de (B.1) são aqueles que satisfazem o Caso III do teorema. É imediato que pontos de equilíbrio que satisfazem o Caso III do Teorema B.3.1 têm a propriedade dada por (B.8).

Corolário B.3.3 Suponha $\alpha \in B^{n}$. Se $\beta=A^{-1}(T \alpha+I) \in(C(\alpha))^{0}$ (onde $(C(\alpha))^{0}$ denota o interior do conjunto $C(\alpha)$ ), então $\beta$ é um ponto de equilíbrio assintoticamente estável de (B.1).

Define-se, a seguir, uma solução para (B.1).

Definição B.3.4 Para algum $\xi \in \Lambda_{m}$, a função $\varphi:(0, \delta) \rightarrow C(\xi)$ de classe $C^{1}$ é dita uma solução local de (B.1) (ou, equivalentemente, de (B.4)) se ela é solução do sistema linear:

$$
\dot{\varphi}(t)=-A_{i} \varphi_{I}(t)+T_{I, I} \varphi_{I}(t)+T_{I, I I} \xi_{I I}+I_{I}, \quad \text { para } t \in(0, \delta)
$$


e se

$$
\min \left(x_{\xi I I} \cdot \xi_{I I}\right) \geq 1, \quad \text { para } t \in(0, \delta)
$$

onde $\varphi_{I}=\left[\varphi_{\sigma(1)}, \ldots, \varphi_{\sigma(m)}\right]^{T}, \varphi_{I I}=\left[\varphi_{\sigma(m+1)}, \ldots, \varphi_{\sigma(n)}\right]^{T}$ e $x_{\xi I I}=A^{-1}\left(T_{I I, I} \varphi_{I I}(t)+\right.$ $\left.T_{I I, I I} \xi_{I I}+I_{I I}\right)$.

A seguir, define-se uma função de energia para a RNC.

Definição B.3.5 Seja $T \in R^{n \times n}$ simétrica e seja $E: \mathbb{R}^{n} \rightarrow \mathbb{R}$ contínua, definida por:

$$
E(y)=-\frac{1}{2} y^{T} T y+\frac{1}{2} y^{T} y-y^{T} I
$$

A função $E(y)$, assim definida, é dita uma função de energia do sistema (B.1).

A partir dessas definições, tem-se o seguinte resultado acerca da estabilidade de soluções de (B.1).

Teorema B.3.6 Suponha que (B.1) satisfaça as Suposições (H1) e (H2). Então, pode-se afirmar que:

1) A função de energia (B.10) decresce monoticamente, ao longo de soluções de (B.1);

2) Toda solução de (B.1) converge para um ponto de equilíbrio.

Prova: Considere uma solução local não-constante de (B.1), dada por $\varphi:(0, \delta) \rightarrow C(\xi)$, $\xi \in \Lambda_{m}$, como na Definição B.3.4. Com a notação dada em (B.3), tem-se que, em $(0, \delta)$

$$
\begin{aligned}
E(\varphi(t))= & -\frac{1}{2} \varphi(t)^{T} \cdot T \cdot \varphi(t)+\frac{1}{2} \varphi(t)^{T} \varphi(t)-\varphi(t)^{T} \cdot I \\
= & -\frac{1}{2}\left(\varphi_{I}(t)^{T}, \varphi_{I I}(t)^{T}\right) \cdot\left[\begin{array}{cc}
T_{I, I} & T_{I, I I} \\
T_{I I, I} & T_{I I, I I}
\end{array}\right] \cdot\left[\begin{array}{c}
\varphi_{I}(t) \\
\varphi_{I I}(t)
\end{array}\right]+ \\
& +\frac{1}{2}\left(\varphi_{I}^{T}(t), \varphi_{I I}^{T}(t)\right) \cdot\left[\begin{array}{c}
\varphi_{I}(t) \\
\varphi_{I I}(t)
\end{array}\right]-\left(\varphi_{I}^{T}(t), \varphi_{I I}^{T}(t)\right) \cdot\left[\begin{array}{c}
I_{I}(t) \\
I_{I I}(t)
\end{array}\right] \\
= & -\frac{1}{2}\left(\varphi_{I}^{T}(t) \cdot T_{I, I} \cdot \varphi_{I}(t)+2 \cdot \varphi_{I}^{T}(t) \cdot T_{I, I I} \cdot \xi_{I I}+\xi_{I I}^{T} \cdot \xi_{I I}\right)+ \\
& +\frac{1}{2}\left(\varphi_{I}^{T}(t) \cdot \varphi_{I}(t)+\xi_{I I}^{T} \cdot \xi_{I I}\right)-\left(\varphi_{I}^{T}(t) \cdot I_{I}+\xi_{I I}^{T} \cdot I_{I I}\right)
\end{aligned}
$$

Por (B.9), e sabendo que $\varphi_{I I}=\xi_{I I}$, tem-se 


$$
\begin{aligned}
\frac{d}{d t} E \varphi(t) & =\nabla E \cdot \frac{d}{d t} \varphi_{I}(t)+\nabla E \cdot \frac{d}{d t} \varphi_{I I}(t) \\
& =-\left(T_{I, I} \cdot \varphi_{I}(t)+T_{I, I I} \cdot \xi_{I I}-\varphi_{I}(t)+I_{I}\right) \cdot \frac{d}{d t} \varphi_{I}(t) \\
& =\frac{d}{d t} \varphi(t)^{2}<0
\end{aligned}
$$

o que prova a Parte 1) do teorema. A Parte 2) segue da teoria de equações diferenciais ordinárias. 


\section{Apêndice C}

\section{Decomposição por Valor Singular}

A decomposição de $A \in \mathbb{R}^{m \times n}$ na forma

$$
A=U \Sigma V^{T}
$$

onde $U$ e $V$ são matrizes ortogonais, recebe o nome de Decomposição por Valor Singular (Singular Value Decomposition ou $S V D$ ). Essa denominação segue do fato que $\Sigma \in \mathbb{R}^{m \times n}$ é da forma

$$
\Sigma=\left[\begin{array}{ll}
D & 0 \\
0 & 0
\end{array}\right]
$$

onde os zeros representam matrizes nulas e onde $D=\operatorname{diag}\left(\sigma_{1}, \ldots, \sigma_{d}\right) \in \mathbb{R}^{d \times d}$ é uma matriz diagonal, contendo os valores singulares de $A^{T} A$ na diagonal principal. Os valores singulares de $A^{T} A$ são definidos por

$$
\sigma_{i}=\sqrt{\lambda_{i}}, \quad \text { para } 1 \leq i \leq d
$$

onde $\lambda_{i}$ são os autovalores de $A^{T} A$ e $d=\operatorname{posto}(A)$.

As matrizes $U=\left[u_{1}, \ldots, u_{m}\right] \in \mathbb{R}^{m \times m}$ e $V=\left[v_{1}, \ldots, v_{n}\right] \in \mathbb{R}^{n \times n}$ em (C.1) são formadas pelos autovetores $v_{i}, \quad 1 \leq i \leq d$ de $A^{T} A$, arranjados de tal forma que os correspondentes autovalores satisfaçam

$$
\lambda_{1} \geq \cdots \geq \lambda_{n}
$$

e onde

$$
u_{i}=\frac{1}{\sigma_{i}} A v_{i}, \quad \text { para } 1 \leq i \leq d
$$

supondo-se que os valores de singulares $\sigma_{i}, 1 \leq i \leq d$ são não-nulos. 
Pode-se escrever as matrizes $U$ e $V$ como

$$
U=\left[U_{1} \mid U_{2}\right] \text { e } V=\left[V_{1} \mid V_{2}\right]
$$

onde $U_{1}=\left[u_{1}, \ldots, u_{d}\right], U_{2}=\left[u_{d+1}, \ldots, u_{m}\right], V_{1}=\left[v_{1}, \ldots, v_{d}\right]$ e $V_{2}=\left[v_{d+1}, \ldots, v_{n}\right]$, e onde $d$ é o posto de $A$.

As seguintes propriedades podem então ser enunciadas:

\section{Propriedades:}

P1. O conjunto $\left\{u_{1}, \ldots, u_{d}\right\}$ (formado pelas colunas de $U_{1}$ ) constitui uma base ortonormal para $\operatorname{Col} A$ (subespaço vetorial gerado pelos vetores-coluna de $A$ );

P2. O conjunto $\left\{u_{d+1}, \ldots, u_{m}\right\}$ (formado pelas colunas de $U_{2}$ ) constitui uma base ortonormal para $(\operatorname{Col} A)^{\perp}$ (subespaço vetorial perpendicular a $C o l A$ ).

Uma importante aplicação da decomposição por valor singular é a derivação da seguinte fórmula para a matriz pseudo-inversa de $A$ :

$$
A^{+}=V_{1} D^{-1} U_{1}^{T}
$$

que é utilizada na Seção 6.3, como parte do método de treinamento de RNCs. 


\section{Apêndice D}

\section{Prova do Teorema 6.6.1}

Seja $y^{0} \in B^{n}$ satisfazendo $y_{i}^{0} \neq y_{i}^{*}$, isto é, $y_{i}^{0}=-y_{i}^{*}$ e $\sum_{j \in Z_{i}}\left|y_{j}^{0}-y_{j}^{*}\right|<\kappa_{i}$ e seja $\delta=y^{0}-y^{*}$.

Tem-se que

$$
\begin{aligned}
\left|\sum_{j \in Z_{i}} T_{i j} \delta_{j}\right| & =\left|\sum_{j \in Z_{i}} T_{i j}\left(y_{j}^{0}-y_{j}^{*}\right)\right| \\
& \leq \max _{j \in Z_{i}}\left|T_{i j}\right|\left(\sum_{j \in Z_{i}}\left|y_{j}^{0}-y_{j}^{*}\right|\right) \\
& \leq \kappa_{i} \max _{j \in Z_{i}}\left|T_{i j}\right|
\end{aligned}
$$

e, portanto

$$
\begin{aligned}
y_{i}^{0}\left(\sum_{j=1}^{n} T_{i j} y_{j}^{0}+I_{i}\right) & =y_{i}^{0}\left(T_{i i} y_{i}^{0}+\sum_{j \in Z_{i}} T_{i j}+I_{i}\right) \\
& =T_{i i}+y_{i}^{0}\left(\sum_{j \in Z_{i}} T_{i j}+I_{i}\right) \\
& =T_{i i}-y_{i}^{*}\left(\sum_{j \in Z_{i}} T i j y_{i}^{*}+I_{i}+\sum_{j \in Z_{i}} T_{i j} \delta_{j}\right) \\
& \leq T_{i i}-y_{i}^{*}\left(\sum_{j \in Z_{i}} T_{i j} y^{*} j+I_{i}\right)+\left|\sum_{j \in Z_{i}} T_{i j} \delta_{j}\right| \\
& \leq T_{i i}-y_{i}^{*}\left(\sum_{j \in Z_{i}} T_{i j} y_{j}^{*}+I_{i}\right)+\kappa_{i} \max _{j \in Z_{i}}\left|T_{i j}\right| \\
& =-\left[y_{i}^{*}\left(\sum_{j \in Z_{i}} T_{i j} y_{j}^{*}+I_{i}\right)-\kappa_{i} \max _{j \in Z_{i}}\left|T_{i j}\right|-\left(T_{i i}-1\right)\right]+1 .
\end{aligned}
$$

Do resultado anterior, e de (6.32), tem-se que 


$$
y^{0}\left(\sum_{j=1}^{n} T_{i j} y_{j}^{0}+I_{i}\right)<1
$$

Agora, considere a condição inicial $x(0)=y^{0}$ para o sistema (6.1). Como

$$
\begin{aligned}
\dot{x}_{i}(0) & =-x_{i}(0)+\sum_{j=1}^{n} T_{i j} \operatorname{sat}\left(x_{j}(0)\right)+I_{i} \\
& =-y_{i}^{0}+\sum_{j=1}^{n} T_{i j}+I_{i}=y_{i}^{*}+\sum_{j=1}^{n} T_{i j}+I_{i},
\end{aligned}
$$

tem-se que

$$
y_{i}^{*} x_{i}(0)=y_{i}^{*}\left(y_{i}^{*}+\sum_{j=1}^{n} T_{i j}+I_{i}\right)=1-y_{i}^{0}\left(\sum_{j=1}^{n} T_{i j}+I_{i}\right)>0 .
$$

A inequação anterior mostra que $y_{i}^{*}$ e $\dot{x}_{i}(0)$ têm o mesmo sinal, o que prova a Parte (a) do teorema. Finalmente, como (D.1) viola a condição (6.29), pode-se concluir que a Parte (b) é verdadeira. Isto completa a prova do teorema. 


\section{Apêndice E}

\section{Tabelas de resultados dos experimentos do Capítulo 7}

As tabelas que seguem, contém os resultados do teste de desempenho apresentado no Capítulo 7. Para ruído binário, a coluna Ruído indica a porcentagem de pixels (ou bits) que foram invertidos nas imagens da Figura 7.1. Para o teste com ruído gaussiano, o valor encontrado na coluna Ruído denota o desvio padrão da distribuição normal com média zero, utilizada para gerar tal ruído. A coluna Carga, por sua vez, denota a razão do número de padrões armazenados pelo tamanho da rede. As colunas que levam o símbolo $\mu$, denotam a taxa média de recuperação de padrões pelos métodos indicados, sendo que os valores estão entre 0.0 e 1.0, representando a razão do número de padrões corretamente recuperados pela quantidade de tentativas de recuperação. As colunas denotadas por $\sigma$, por sua vez, indicam os desvios padrões medidos.

Considere a seguinte legenda nas tabelas que seguem: P-Inversa denota o método da Seção 6.2; Perceptron corresponde ao método da Seção 6.5; SVD indica o método da Seção 6.3; Hebb denota o método da Seção 6.4 e LMI referencia o método da Seção 6.6. 


\section{E.1 Tabelas de resultados para ruído binário}

As tabelas à seguir resumem os resultados dos experimentos realizados com padrões corrompidos por ruído binário.

Tabela E.1: Desempenho dos métodos para padrões com ruído binário e vizinhança $r=1$.

\begin{tabular}{|c|c|c|c|c|c|c|c|c|c|c|c|}
\hline \multicolumn{2}{|c|}{ Níveis de } & \multicolumn{2}{|c|}{ P-Inv } & \multicolumn{2}{|c|}{ Percept } & \multicolumn{2}{|c|}{ SVD } & \multicolumn{2}{|c|}{ Hebb } & \multicolumn{2}{|c|}{ LMI } \\
\hline Carga & Ruído & $\mu$ & $\sigma$ & $\mu$ & $\sigma$ & $\mu$ & $\sigma$ & $\mu$ & $\sigma$ & $\mu$ & $\sigma$ \\
\hline \multirow{6}{*}{$10 \%$} & $2 \%$ & .712 & .200 & 0.527 & 0.182 & .712 & 0.188 & .939 & 0.084 & 0.873 & .109 \\
\hline & $4 \%$ & 506 & 0.287 & 0.282 & 0.142 & 0.518 & 0.236 & 0.842 & 0.143 & 0.727 & 0.136 \\
\hline & $6 \%$ & 0.391 & 0.266 & 0.185 & 0.146 & 0.427 & 0.282 & 0.779 & 0.158 & 0.676 & 0.230 \\
\hline & $10 \%$ & 0.248 & 0.295 & 0.073 & 0.096 & 0.239 & 0.235 & 0.627 & 0.243 & 0.515 & 0.230 \\
\hline & $15 \%$ & 0.121 & 0.207 & 0.012 & 0.027 & 0.097 & 0.157 & 0.373 & 0.261 & 0.342 & 0.246 \\
\hline & $20 \%$ & 0.067 & 0.107 & 0.003 & 0.010 & 0.064 & 0.090 & 0.258 & 0.182 & 0.218 & 0.219 \\
\hline \multirow{6}{*}{$20 \%$} & $2 \%$ & 0.336 & 0.139 & 0.318 & 0.108 & 0.379 & 0.132 & 0.815 & 0.130 & 0.739 & 0.154 \\
\hline & $4 \%$ & 0.118 & 0.119 & 0.100 & 0.073 & 0.139 & 0.139 & 0.645 & 0.141 & 0.533 & 0.130 \\
\hline & $6 \%$ & 0.027 & 0.025 & 0.045 & 0.031 & 0.042 & 0.052 & 0.488 & 0.104 & 0.352 & 0.120 \\
\hline & $10 \%$ & 0.006 & 0.013 & 0.000 & 0.000 & 0.006 & 0.020 & 0.245 & 0.114 & 0.264 & 0.089 \\
\hline & $15 \%$ & 0.000 & 0.000 & 0.000 & 0.000 & 0.000 & 0.000 & 0.097 & 0.124 & 0.088 & 0.097 \\
\hline & $20 \%$ & 0.000 & 0.000 & 0.000 & 0.000 & 0.000 & 0.000 & 0.033 & 0.033 & 0.027 & 0.029 \\
\hline \multirow{6}{*}{$30 \%$} & $2 \%$ & $\overline{0.194}$ & 0.155 & 0.245 & $\overline{0.104}$ & $\overline{0.197}$ & 0.157 & 0.645 & 0.203 & 0.639 & 0.142 \\
\hline & $4 \%$ & 0.039 & 0.057 & 0.039 & 0.042 & 0.052 & 0.081 & 0.382 & 0.170 & 0.388 & 0.152 \\
\hline & $6 \%$ & 0.009 & 0.022 & 0.009 & 0.022 & 0.015 & 0.035 & 0.288 & 0.149 & 0.264 & 0.129 \\
\hline & $10 \%$ & 0.000 & 0.000 & 0.000 & 0.000 & 0.000 & 0.000 & 0.091 & 0.116 & 0.145 & 0.096 \\
\hline & $15 \%$ & 0.000 & 0.000 & 0.000 & 0.000 & 0.000 & 0.000 & 0.048 & 0.129 & 0.058 & 0.096 \\
\hline & $20 \%$ & 0.000 & 0.000 & 0.000 & 0.000 & 0.000 & 0.000 & 0.012 & 0.027 & 0.018 & 0.027 \\
\hline \multirow{6}{*}{$40 \%$} & $2 \%$ & 0.112 & 0.062 & 0.203 & 0.050 & 0.109 & 0.060 & 0.509 & 0.159 & 0.609 & 0.133 \\
\hline & $4 \%$ & 0.006 & 0.013 & 0.055 & 0.040 & 0.006 & 0.013 & 0.230 & 0.102 & 0.342 & 0.113 \\
\hline & $6 \%$ & 0.000 & 0.000 & 0.015 & 0.023 & 0.000 & 0.000 & 0.127 & 0.107 & 0.200 & 0.082 \\
\hline & $10 \%$ & 0.000 & 0.000 & 0.000 & 0.000 & 0.000 & 0.000 & 0.027 & 0.051 & 0.097 & 0.055 \\
\hline & $15 \%$ & 0.000 & 0.000 & 0.000 & 0.000 & 0.000 & 0.000 & 0.000 & 0.000 & 0.018 & 0.040 \\
\hline & $20 \%$ & 0.000 & 0.000 & 0.000 & 0.000 & 0.000 & 0.000 & 0.000 & 0.000 & 0.027 & 0.039 \\
\hline \multirow{6}{*}{$50 \%$} & $2 \%$ & 0.061 & 0.059 & 0.170 & 0.078 & 0.048 & 0.052 & 0.415 & 0.183 & 0.576 & 0.104 \\
\hline & $4 \%$ & 0.006 & 0.013 & 0.042 & 0.045 & 0.006 & 0.013 & 0.167 & 0.105 & 0.385 & 0.109 \\
\hline & $6 \%$ & 0.000 & 0.000 & 0.006 & 0.013 & 0.000 & 0.000 & 0.067 & 0.076 & 0.197 & 0.077 \\
\hline & $10 \%$ & 0.000 & 0.000 & 0.000 & 0.000 & 0.000 & 0.000 & 0.021 & 0.027 & 0.112 & 0.090 \\
\hline & $15 \%$ & 0.000 & 0.000 & 0.000 & 0.000 & 0.000 & 0.000 & 0.003 & 0.010 & 0.055 & 0.062 \\
\hline & $20 \%$ & 0.000 & 0.000 & 0.000 & 0.000 & 0.000 & 0.000 & 0.003 & 0.010 & 0.018 & 0.031 \\
\hline
\end{tabular}


Tabela E.2: Desempenho dos métodos para padrões com ruído binário e vizinhança r=2.

\begin{tabular}{|c|c|c|c|c|c|c|c|c|c|c|c|}
\hline \multicolumn{2}{|c|}{ Níveis de } & \multicolumn{2}{|c|}{ P-Inv } & \multicolumn{2}{|c|}{ Percept } & \multicolumn{2}{|c|}{ SVD } & \multicolumn{2}{|c|}{ Hebb } & \multicolumn{2}{|c|}{ LMI } \\
\hline Carga & Ruído & $\mu$ & $\sigma$ & $\mu$ & $\sigma$ & $\mu$ & $\sigma$ & $\mu$ & $\sigma$ & $\mu$ & $\sigma$ \\
\hline \multirow{6}{*}{$10 \%$} & $2 \%$ & .945 & "0.083 & 8.897 & "0.080 & 0.985 & 1.031 & "0.997 & $\overline{c 0.010}$ & 1.000 & "0.000 \\
\hline & $4 \%$ & 0.888 & 0.133 & .779 & 0.175 & 0.891 & .106 & 0.988 & 0.027 & 0.982 & 0.035 \\
\hline & $6 \%$ & 0.879 & 0.146 & 0.682 & 0.188 & 0.824 & 0.117 & 0.988 & 0.022 & 0.979 & 0.052 \\
\hline & $10 \%$ & 0.779 & 0.234 & 0.545 & 0.246 & 0.618 & 0.222 & 0.979 & 0.043 & 0.964 & 0.064 \\
\hline & $15 \%$ & 0.636 & 0.298 & 0.364 & 0.279 & 0.318 & 0.225 & 0.933 & 0.068 & 0.967 & 0.056 \\
\hline & $20 \%$ & 0.615 & 0.249 & 0.336 & 0.240 & 0.206 & 0.153 & 0.915 & 0.099 & 0.903 & 0.116 \\
\hline \multirow{6}{*}{$20 \%$} & $2 \%$ & 0.824 & 0.104 & 0.758 & 0.088 & 0.842 & 0.121 & 0.988 & 0.017 & 0.985 & 0.027 \\
\hline & $4 \%$ & 0.703 & 0.140 & 0.570 & 0.135 & 0.624 & 0.187 & 0.979 & 0.022 & 0.970 & 0.038 \\
\hline & $6 \%$ & 0.588 & 0.139 & 0.479 & 0.142 & 0.518 & 0.172 & 0.973 & 0.042 & 0.948 & 0.075 \\
\hline & $10 \%$ & 0.364 & 0.157 & 0.227 & 0.129 & 0.248 & 0.157 & 0.903 & 0.060 & 0.891 & 0.116 \\
\hline & $15 \%$ & 0.182 & 0.191 & 0.100 & 0.071 & 0.061 & 0.073 & 0.794 & 0.105 & 0.836 & 0.122 \\
\hline & $20 \%$ & 0.124 & 0.202 & 0.082 & 0.079 & 0.030 & 0.053 & 0.697 & 0.135 & 0.745 & 0.144 \\
\hline \multirow{6}{*}{$30 \%$} & $2 \%$ & 0.688 & 0.147 & 0.636 & 0.113 & 0.576 & 0.180 & 0.976 & 0.037 & 0.973 & 0.029 \\
\hline & $4 \%$ & 0.427 & 0.208 & 0.397 & 0.149 & 0.321 & 0.211 & 0.964 & 0.035 & 0.955 & 0.043 \\
\hline & $6 \%$ & 0.276 & 0.145 & 0.291 & 0.174 & 0.194 & 0.195 & 0.888 & 0.099 & 0.909 & 0.083 \\
\hline & $10 \%$ & 0.121 & 0.142 & 0.133 & 0.097 & 0.073 & 0.131 & 0.785 & 0.130 & 0.858 & 0.139 \\
\hline & $15 \%$ & 0.042 & 0.098 & 0.027 & 0.042 & 0.027 & 0.070 & 0.552 & 0.180 & 0.718 & 0.119 \\
\hline & $20 \%$ & 0.015 & 0.031 & 0.024 & 0.034 & 0.012 & 0.040 & 0.488 & 0.154 & 0.591 & 0.125 \\
\hline \multirow{6}{*}{$40 \%$} & $2 \%$ & 0.530 & 0.183 & 612 & 0.107 & 0.433 & 0.159 & .967 & 0.026 & 0.976 & 0.040 \\
\hline & $4 \%$ & 0.252 & 0.129 & 0.339 & 0.070 & 0.188 & 0.119 & 0.952 & 0.060 & 0.952 & 0.058 \\
\hline & $6 \%$ & 0.161 & 0.113 & 0.239 & 0.061 & 0.091 & 0.100 & 0.867 & 0.094 & 0.909 & 0.076 \\
\hline & $10 \%$ & 0.055 & 0.107 & 0.088 & 0.067 & 0.018 & 0.050 & 0.730 & 0.164 & 0.824 & 0.142 \\
\hline & $15 \%$ & 0.006 & 0.013 & 0.018 & 0.040 & 0.006 & 0.013 & 0.561 & 0.121 & 0.673 & 0.158 \\
\hline & $20 \%$ & 0.003 & 0.010 & 0.012 & 0.017 & 0.000 & 0.000 & 0.348 & 0.160 & 0.509 & 0.136 \\
\hline \multirow{6}{*}{$50 \%$} & $2 \%$ & 0.370 & 0.173 & 588 & 0.086 & 0.312 & 0.190 & 0.936 & 0.048 & 0.958 & 0.037 \\
\hline & $4 \%$ & 0.185 & 0.118 & 0.373 & 0.106 & 0.139 & 0.110 & 0.912 & 0.062 & 0.958 & 0.058 \\
\hline & $6 \%$ & 0.070 & 0.072 & 0.233 & 0.070 & 0.039 & 0.053 & 0.794 & 0.126 & 0.879 & 0.106 \\
\hline & $10 \%$ & 0.012 & 0.022 & 0.048 & 0.038 & 0.003 & 0.010 & 0.670 & 0.135 & 0.791 & 0.110 \\
\hline & $15 \%$ & 0.003 & 0.010 & 0.015 & 0.023 & 0.000 & 0.000 & 0.397 & 0.146 & 0.639 & 0.122 \\
\hline & $20 \%$ & 0.000 & 0.000 & 0.006 & 0.013 & 0.000 & 0.000 & 0.255 & 0.175 & 0.479 & 0.154 \\
\hline
\end{tabular}


Tabela E.3: Desempenho dos métodos para padrões com ruído binário e vizinhança $\mathrm{r}=3$.

\begin{tabular}{|c|c|c|c|c|c|c|c|c|c|c|c|}
\hline \multicolumn{2}{|c|}{ Níveis de } & \multicolumn{2}{|c|}{ P-Inv } & \multicolumn{2}{|c|}{ Percept } & \multicolumn{2}{|c|}{ SVD } & \multicolumn{2}{|c|}{ Hebb } & \multicolumn{2}{|c|}{ LMI } \\
\hline Carga & Ruído & $\mu$ & $\sigma$ & $\mu$ & $\sigma$ & $\mu$ & $\sigma$ & $\mu$ & $\sigma$ & $\mu$ & $\sigma$ \\
\hline \multirow{6}{*}{$10 \%$} & $2 \%$ & $\overline{c 0.830}$ & 0.285 & "0.976 & $\overline{c 0.042}$ & "0.994 & "0.020 & 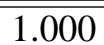 & "0.000 & 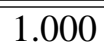 & $\overline{0.000}$ \\
\hline & $4 \%$ & 0.821 & 0.273 & 0.903 & 0.096 & 0.973 & 0.029 & 1.000 & 0.000 & 1.000 & 0.000 \\
\hline & $6 \%$ & 0.818 & 0.286 & 0.870 & 0.117 & 0.897 & 0.086 & 1.000 & 0.000 & 0.997 & 0.010 \\
\hline & $10 \%$ & 0.794 & 0.280 & 0.812 & 0.161 & 0.673 & 0.165 & 0.997 & 0.010 & 1.000 & 0.000 \\
\hline & $15 \%$ & 0.755 & 0.332 & 0.673 & 0.249 & 0.300 & 0.120 & 0.991 & 0.022 & 0.994 & 0.020 \\
\hline & $20 \%$ & 0.733 & 0.330 & 0.624 & 0.207 & 0.167 & 0.076 & 0.982 & 0.035 & 0.991 & 0.022 \\
\hline \multirow{6}{*}{$20 \%$} & $2 \%$ & 0.748 & 0.191 & 0.900 & 0.071 & 0.961 & 0.051 & 0.997 & 0.010 & 0.997 & 0.010 \\
\hline & $4 \%$ & 0.706 & 0.179 & 0.776 & 0.087 & 0.903 & 0.060 & 0.994 & 0.020 & 0.994 & 0.013 \\
\hline & $6 \%$ & 0.679 & 0.233 & 0.667 & 0.105 & 0.745 & 0.096 & 0.997 & 0.010 & 0.991 & 0.022 \\
\hline & $10 \%$ & 0.582 & 0.222 & 0.552 & 0.112 & 0.506 & 0.132 & 0.991 & 0.022 & 0.988 & 0.031 \\
\hline & $15 \%$ & 0.485 & 0.242 & 0.385 & 0.149 & 0.167 & 0.058 & 0.979 & 0.031 & 0.979 & 0.040 \\
\hline & $20 \%$ & 0.391 & 0.260 & 0.282 & 0.179 & 0.073 & 0.059 & 0.948 & 0.069 & 0.952 & 0.074 \\
\hline \multirow{6}{*}{$30 \%$} & $2 \%$ & 0.597 & 0.281 & 0.864 & 0.075 & 0.858 & 0.092 & 1.000 & 0.000 & 0.991 & 0.022 \\
\hline & $4 \%$ & 0.542 & 0.254 & 0.679 & 0.134 & 0.679 & 0.159 & 0.997 & 0.010 & 0.991 & 0.022 \\
\hline & $6 \%$ & 0.427 & 0.227 & 0.573 & 0.120 & 0.521 & 0.177 & 0.985 & 0.031 & 0.988 & 0.022 \\
\hline & $10 \%$ & 0.339 & 0.190 & 0.376 & 0.148 & 0.261 & 0.148 & 0.955 & 0.048 & 0.982 & 0.035 \\
\hline & $15 \%$ & 0.167 & 0.181 & 0.194 & 0.181 & 0.076 & 0.072 & 0.927 & 0.087 & 0.948 & 0.038 \\
\hline & $20 \%$ & 0.118 & 0.173 & 0.112 & 0.100 & 0.021 & 0.034 & 0.845 & 0.098 & 0.903 & 0.078 \\
\hline \multirow{6}{*}{$40 \%$} & $2 \%$ & 0.415 & 0.234 & 0.770 & 0.077 & 0.767 & 0.109 & 1.000 & 0.000 & 0.991 & 0.022 \\
\hline & $4 \%$ & 0.312 & 0.215 & 0.606 & 0.083 & 0.494 & 0.132 & 0.991 & 0.016 & 0.979 & 0.043 \\
\hline & $6 \%$ & 0.233 & 0.189 & 0.506 & 0.087 & 0.352 & 0.153 & 0.970 & 0.031 & 0.982 & 0.035 \\
\hline & $10 \%$ & 0.170 & 0.132 & 0.352 & 0.112 & 0.215 & 0.143 & 0.915 & 0.072 & 0.948 & 0.052 \\
\hline & $15 \%$ & 0.070 & 0.074 & 0.152 & 0.091 & 0.048 & 0.048 & 0.891 & 0.076 & 0.924 & 0.052 \\
\hline & $20 \%$ & 0.036 & 0.067 & 0.076 & 0.047 & 0.000 & 0.000 & 0.773 & 0.110 & 0.852 & 0.095 \\
\hline \multirow{6}{*}{$50 \%$} & $2 \%$ & 0.306 & 0.218 & 0.745 & 0.089 & 0.642 & 0.138 & 0.994 & 0.013 & 0.994 & 0.013 \\
\hline & $4 \%$ & 0.242 & 0.195 & 0.603 & 0.094 & 0.433 & 0.137 & 0.970 & 0.050 & 0.982 & 0.035 \\
\hline & $6 \%$ & 0.148 & 0.134 & 0.488 & 0.069 & 0.236 & 0.131 & 0.961 & 0.039 & 0.985 & 0.027 \\
\hline & $10 \%$ & 0.076 & 0.107 & 0.248 & 0.092 & 0.103 & 0.125 & 0.924 & 0.067 & 0.973 & 0.036 \\
\hline & $15 \%$ & 0.030 & 0.041 & 0.097 & 0.080 & 0.033 & 0.047 & 0.806 & 0.103 & 0.915 & 0.079 \\
\hline & $20 \%$ & 0.009 & 0.022 & 0.048 & 0.035 & 0.003 & 0.010 & 0.700 & 0.148 & 0.818 & 0.091 \\
\hline
\end{tabular}




\section{E.2 Tabelas de resultados para ruído gaussiano}

As tabelas à seguir resumem os resultados dos experimentos realizados com padrões corrompidos com ruído gaussiano.

Tabela E.4: Desempenho dos métodos para padrões com ruído gaussiano e vizinhança $r=1$.

\begin{tabular}{|c|c|c|c|c|c|c|c|c|c|c|c|}
\hline \multicolumn{2}{|c|}{ Níveis de } & \multicolumn{2}{|c|}{ P-Inv } & \multicolumn{2}{|c|}{ Percept } & \multicolumn{2}{|c|}{ SVD } & \multicolumn{2}{|c|}{ Hebb } & \multicolumn{2}{|c|}{ LMI } \\
\hline Carga & Ruído & $\mu$ & $\sigma$ & $\mu$ & $\sigma$ & $\mu$ & $\sigma$ & $\mu$ & $\sigma$ & $\mu$ & $\sigma$ \\
\hline \multirow{6}{*}{$10 \%$} & 0.3 & 1.000 & .000 & 1.000 & 0.000 & 1.000 & 0.000 & 0.979 & 0.027 & 0.488 & 0.300 \\
\hline & 0.5 & 0.855 & .131 & 0.900 & 0.084 & 0.882 & 0.115 & 0.933 & 0.093 & 0.518 & 0.275 \\
\hline & 0.7 & 0.552 & 0.268 & 0.561 & 0.174 & 0.564 & 0.239 & 0.776 & 0.154 & 0.467 & 0.299 \\
\hline & 1.0 & 0.221 & 0.205 & 0.191 & 0.129 & 0.233 & 0.191 & 0.479 & 0.227 & 0.367 & 0.270 \\
\hline & 1.5 & 0.042 & 0.058 & 0.015 & 0.023 & 0.045 & 0.065 & 0.191 & 0.127 & 0.158 & 0.136 \\
\hline & 2.0 & 0.009 & 0.030 & 0.006 & 0.013 & 0.012 & 0.017 & 0.082 & 0.067 & 0.091 & 0.083 \\
\hline \multirow{6}{*}{$20 \%$} & 0.3 & 1.000 & 0.000 & 0.988 & 0.017 & 1.000 & 0.000 & 0.973 & 0.033 & 0.233 & 0.113 \\
\hline & 0.5 & 0.664 & 0.097 & 0.830 & 0.067 & 0.685 & 0.091 & 0.903 & 0.053 & 0.291 & 0.156 \\
\hline & 0.7 & 0.191 & 0.102 & 0.382 & 0.090 & 0.197 & 0.108 & 0.624 & 0.083 & 0.197 & 0.105 \\
\hline & 1.0 & 0.027 & 0.042 & 0.045 & 0.043 & 0.033 & 0.052 & 0.188 & 0.109 & 0.100 & 0.068 \\
\hline & 1.5 & 0.003 & 0.010 & 0.000 & 0.000 & 0.003 & 0.010 & 0.027 & 0.033 & 0.048 & 0.048 \\
\hline & 2.0 & 0.000 & 0.000 & 0.000 & 0.000 & 0.000 & 0.000 & 0.015 & 0.023 & 0.015 & 0.023 \\
\hline \multirow{6}{*}{$30 \%$} & $\overline{0.3}$ & 0.988 & 0.017 & 0.991 & 0.022 & 0.985 & 0.017 & 0.982 & 0.027 & 0.124 & $\overline{0.076}$ \\
\hline & 0.5 & 0.500 & 0.146 & 0.736 & 0.094 & 0.494 & 0.150 & 0.858 & 0.070 & 0.136 & 0.086 \\
\hline & 0.7 & 0.097 & 0.084 & 0.291 & 0.098 & 0.100 & 0.086 & 0.476 & 0.120 & 0.121 & 0.073 \\
\hline & 1.0 & 0.003 & 0.010 & 0.027 & 0.036 & 0.006 & 0.020 & 0.127 & 0.139 & 0.064 & 0.064 \\
\hline & 1.5 & 0.000 & 0.000 & 0.000 & 0.000 & 0.000 & 0.000 & 0.003 & 0.010 & 0.015 & 0.023 \\
\hline & 2.0 & 0.000 & 0.000 & 0.000 & 0.000 & 0.000 & 0.000 & 0.009 & 0.022 & 0.009 & 0.016 \\
\hline \multirow{6}{*}{$40 \%$} & 0.3 & 0.979 & 0.022 & 0.979 & 0.022 & 0.979 & 0.022 & 0.988 & 0.022 & 0.106 & 0.092 \\
\hline & 0.5 & 0.415 & 0.090 & 0.700 & 0.060 & 0.421 & 0.092 & 0.803 & 0.069 & 0.112 & 0.081 \\
\hline & 0.7 & 0.067 & 0.056 & 0.273 & 0.102 & 0.067 & 0.056 & 0.409 & 0.108 & 0.088 & 0.078 \\
\hline & 1.0 & 0.000 & 0.000 & 0.024 & 0.034 & 0.000 & 0.000 & 0.061 & 0.039 & 0.048 & 0.056 \\
\hline & 1.5 & 0.000 & 0.000 & 0.000 & 0.000 & 0.000 & 0.000 & 0.003 & 0.010 & 0.009 & 0.022 \\
\hline & 2.0 & 0.000 & 0.000 & 0.000 & 0.000 & 0.000 & 0.000 & 0.003 & 0.010 & 0.006 & 0.013 \\
\hline \multirow{6}{*}{$50 \%$} & 0.3 & 0.976 & 0.022 & 0.988 & 0.017 & 0.976 & 0.022 & 0.988 & 0.031 & 0.124 & 0.119 \\
\hline & 0.5 & 0.424 & 0.058 & 0.703 & 0.090 & 0.415 & 0.052 & 0.800 & 0.033 & 0.097 & 0.089 \\
\hline & 0.7 & 0.048 & 0.043 & 0.191 & 0.101 & 0.048 & 0.043 & 0.312 & 0.146 & 0.076 & 0.068 \\
\hline & 1.0 & 0.003 & 0.010 & 0.018 & 0.023 & 0.003 & 0.010 & 0.045 & 0.031 & 0.039 & 0.039 \\
\hline & 1.5 & 0.000 & 0.000 & 0.000 & 0.000 & 0.000 & 0.000 & 0.000 & 0.000 & 0.015 & 0.027 \\
\hline & 2.0 & 0.000 & 0.000 & 0.000 & 0.000 & 0.000 & 0.000 & 0.000 & 0.000 & 0.009 & 0.016 \\
\hline
\end{tabular}


Tabela E.5: Desempenho dos métodos para padrões com ruído gaussiano e vizinhança $\mathrm{r}=2$.

\begin{tabular}{|c|c|c|c|c|c|c|c|c|c|c|c|}
\hline \multicolumn{2}{|c|}{ Níveis de } & \multicolumn{2}{|c|}{ P-Inv } & \multicolumn{2}{|c|}{ Percept } & \multicolumn{2}{|c|}{ SVD } & \multicolumn{2}{|c|}{ Hebb } & \multicolumn{2}{|c|}{ LMI } \\
\hline Carga & Ruído & $\mu$ & $\sigma$ & $\mu$ & $\sigma$ & $\mu$ & $\sigma$ & $\mu$ & $\sigma$ & $\mu$ & $\sigma$ \\
\hline \multirow{6}{*}{$10 \%$} & 0.3 & 0.964 & 0.081 & 1.000 & 0.000 & 1.000 & 0.000 & 1.000 & 0.000 & 0.964 & 0.086 \\
\hline & 0.5 & 0.948 & 0.079 & 0.988 & 0.022 & 0.988 & 0.022 & 0.997 & 0.010 & 0.970 & 0.074 \\
\hline & 0.7 & 0.915 & 0.127 & 0.891 & 0.130 & 0.879 & 0.122 & 0.985 & 0.035 & 0.961 & 0.095 \\
\hline & 1.0 & 0.755 & 0.240 & 0.670 & 0.228 & 0.506 & 0.184 & 0.939 & 0.100 & 0.936 & 0.146 \\
\hline & 1.5 & 0.485 & 0.275 & 0.270 & 0.197 & 0.155 & 0.097 & 0.791 & 0.145 & 0.794 & 0.122 \\
\hline & 2.0 & 0.336 & 0.326 & 0.173 & 0.159 & 0.048 & 0.054 & 0.652 & 0.213 & 0.679 & 0.178 \\
\hline \multirow{6}{*}{$20 \%$} & 0.3 & 0.988 & 0.022 & 1.000 & 0.000 & 1.000 & 0.000 & 1.000 & 0.000 & 0.933 & 0.104 \\
\hline & 0.5 & 0.915 & 0.091 & 0.973 & 0.020 & 0.942 & 0.056 & 0.991 & 0.022 & 0.930 & 0.106 \\
\hline & 0.7 & 0.730 & 0.113 & 0.773 & 0.107 & 0.679 & 0.106 & 0.970 & 0.041 & 0.930 & 0.091 \\
\hline & 1.0 & 0.336 & 0.209 & 0.324 & 0.161 & 0.212 & 0.145 & 0.867 & 0.084 & 0.885 & 0.106 \\
\hline & 1.5 & 0.073 & 0.104 & 0.039 & 0.068 & 0.030 & 0.060 & 0.548 & 0.117 & 0.621 & 0.118 \\
\hline & 2.0 & 0.030 & 0.031 & 0.018 & 0.023 & 0.009 & 0.016 & 0.300 & 0.065 & 0.355 & 0.154 \\
\hline \multirow{6}{*}{$30 \%$} & 0.3 & 0.994 & 0.020 & 1.000 & 0.000 & 1.000 & 0.000 & 0.994 & 0.013 & 0.912 & 0.097 \\
\hline & 0.5 & 0.830 & 0.097 & 0.918 & 0.058 & 0.855 & 0.097 & 0.973 & 0.025 & 0.882 & 0.124 \\
\hline & 0.7 & 0.479 & 0.134 & 0.627 & 0.130 & 0.418 & 0.141 & 0.894 & 0.051 & 0.897 & 0.101 \\
\hline & 1.0 & 0.115 & 0.119 & 0.215 & 0.106 & 0.085 & 0.106 & 0.733 & 0.094 & 0.809 & 0.118 \\
\hline & 1.5 & 0.009 & 0.030 & 0.024 & 0.026 & 0.003 & 0.010 & 0.339 & 0.150 & 0.467 & 0.141 \\
\hline & 2.0 & 0.003 & 0.010 & 0.006 & 0.013 & 0.000 & 0.000 & 0.158 & 0.083 & 0.264 & 0.122 \\
\hline \multirow{6}{*}{$40 \%$} & 0.3 & 0.994 & 0.013 & 0.997 & 0.010 & 0.997 & 0.010 & 0.997 & 0.010 & 0.924 & 0.098 \\
\hline & 0.5 & 0.830 & 0.096 & 0.912 & 0.050 & 0.815 & 0.112 & 0.976 & 0.022 & 0.924 & 0.102 \\
\hline & 0.7 & 0.430 & 0.149 & 0.627 & 0.088 & 0.364 & 0.126 & 0.927 & 0.055 & 0.897 & 0.115 \\
\hline & 1.0 & 0.058 & 0.060 & 0.191 & 0.086 & 0.039 & 0.068 & 0.697 & 0.103 & 0.791 & 0.117 \\
\hline & 1.5 & 0.006 & 0.013 & 0.015 & 0.027 & 0.000 & 0.000 & 0.291 & 0.132 & 0.452 & 0.142 \\
\hline & 2.0 & 0.003 & 0.010 & 0.006 & 0.013 & 0.000 & 0.000 & 0.121 & 0.092 & 0.261 & 0.122 \\
\hline \multirow{6}{*}{$50 \%$} & 0.3 & 0.970 & 0.050 & 1.000 & 0.000 & 0.997 & 0.010 & 0.991 & 0.016 & 0.942 & 0.065 \\
\hline & 0.5 & 0.730 & 0.089 & 0.915 & 0.035 & 0.721 & 0.070 & 0.970 & 0.041 & 0.921 & 0.087 \\
\hline & 0.7 & 0.312 & 0.175 & 0.612 & 0.100 & 0.264 & 0.138 & 0.873 & 0.102 & 0.891 & 0.103 \\
\hline & 1.0 & 0.033 & 0.049 & 0.161 & 0.077 & 0.015 & 0.027 & 0.582 & 0.121 & 0.773 & 0.110 \\
\hline & 1.5 & 0.003 & 0.010 & 0.006 & 0.013 & 0.003 & 0.010 & 0.203 & 0.121 & 0.445 & 0.126 \\
\hline & 2.0 & 0.003 & 0.010 & 0.003 & 0.010 & 0.000 & 0.000 & 0.079 & 0.058 & 0.185 & 0.087 \\
\hline
\end{tabular}


Tabela E.6: Desempenho dos métodos para padrões com ruído gaussiano e vizinhança r=3.

\begin{tabular}{|c|c|c|c|c|c|c|c|c|c|c|c|}
\hline \multicolumn{2}{|c|}{ Níveis de } & \multicolumn{2}{|c|}{ P-Inv } & \multicolumn{2}{|c|}{ Percept } & \multicolumn{2}{|c|}{ SVD } & \multicolumn{2}{|c|}{ Hebb } & \multicolumn{2}{|c|}{ LMI } \\
\hline Carga & Ruído & $\mu$ & $\sigma$ & $\mu$ & $\sigma$ & $\mu$ & $\sigma$ & $\mu$ & $\sigma$ & $\mu$ & $\sigma$ \\
\hline \multirow{6}{*}{$10 \%$} & $\overline{0.3}$ & 0.852 & 0.258 & 1.000 & "0.000 & $\overline{1.000}$ & $\overline{c 0.000}$ & 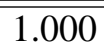 & $\overline{0.000}$ & $\overline{1.000}$ & "0.000 \\
\hline & 0.5 & 0.855 & 0.239 & 0.997 & 0.010 & 0.985 & 0.027 & 0.997 & 0.010 & 1.000 & 0.000 \\
\hline & 0.7 & 0.870 & 0.187 & 0.970 & 0.028 & 0.842 & 0.079 & 0.994 & 0.013 & 1.000 & 0.000 \\
\hline & 1.0 & 0.800 & 0.270 & 0.855 & 0.133 & 0.436 & 0.155 & 0.982 & 0.060 & 0.988 & 0.040 \\
\hline & 1.5 & 0.670 & 0.332 & 0.548 & 0.221 & 0.124 & 0.063 & 0.955 & 0.043 & 0.933 & 0.061 \\
\hline & 2.0 & 0.606 & 0.303 & 0.348 & 0.239 & 0.061 & 0.053 & 0.870 & 0.090 & 0.842 & 0.090 \\
\hline \multirow{6}{*}{$20 \%$} & 0.3 & 0.776 & 0.180 & 1.000 & 0.000 & 1.000 & 0.000 & 0.997 & 0.010 & 0.988 & 0.040 \\
\hline & 0.5 & 0.745 & 0.208 & 0.985 & 0.023 & 0.976 & 0.034 & 0.997 & 0.010 & 0.991 & 0.030 \\
\hline & 0.7 & 0.700 & 0.213 & 0.879 & 0.081 & 0.818 & 0.089 & 0.991 & 0.016 & 0.985 & 0.040 \\
\hline & 1.0 & 0.536 & 0.211 & 0.564 & 0.165 & 0.336 & 0.078 & 0.982 & 0.031 & 0.973 & 0.047 \\
\hline & 1.5 & 0.303 & 0.192 & 0.221 & 0.127 & 0.082 & 0.064 & 0.894 & 0.053 & 0.906 & 0.053 \\
\hline & 2.0 & 0.176 & 0.133 & 0.094 & 0.088 & 0.012 & 0.022 & 0.645 & 0.090 & 0.676 & 0.084 \\
\hline \multirow{6}{*}{$30 \%$} & 0.3 & 0.703 & 0.214 & 1.000 & 0.000 & 1.000 & 0.000 & 1.000 & 0.000 & 0.985 & 0.035 \\
\hline & 0.5 & 0.642 & 0.240 & 0.964 & 0.038 & 0.958 & 0.034 & 0.988 & 0.022 & 0.988 & 0.027 \\
\hline & 0.7 & 0.458 & 0.146 & 0.773 & 0.090 & 0.697 & 0.123 & 0.979 & 0.027 & 0.988 & 0.022 \\
\hline & 1.0 & 0.297 & 0.157 & 0.397 & 0.156 & 0.206 & 0.115 & 0.936 & 0.048 & 0.967 & 0.039 \\
\hline & 1.5 & 0.094 & 0.098 & 0.118 & 0.062 & 0.015 & 0.023 & 0.718 & 0.138 & 0.776 & 0.079 \\
\hline & 2.0 & 0.039 & 0.059 & 0.055 & 0.090 & 0.000 & 0.000 & 0.430 & 0.127 & 0.515 & 0.075 \\
\hline \multirow{6}{*}{$40 \%$} & 0.3 & 0.597 & 0.182 & 0.997 & 0.010 & 1.000 & 0.000 & 0.997 & 0.010 & 0.982 & 0.043 \\
\hline & 0.5 & 0.500 & 0.211 & 0.964 & 0.028 & 0.964 & 0.041 & 0.991 & 0.016 & 0.973 & 0.061 \\
\hline & 0.7 & 0.400 & 0.164 & 0.785 & 0.090 & 0.661 & 0.125 & 0.967 & 0.049 & 0.979 & 0.040 \\
\hline & 1.0 & 0.152 & 0.082 & 0.376 & 0.119 & 0.167 & 0.105 & 0.924 & 0.056 & 0.948 & 0.050 \\
\hline & 1.5 & 0.042 & 0.056 & 0.082 & 0.056 & 0.015 & 0.027 & 0.594 & 0.160 & 0.700 & 0.118 \\
\hline & 2.0 & 0.015 & 0.027 & 0.027 & 0.033 & 0.000 & 0.000 & 0.382 & 0.152 & 0.524 & 0.101 \\
\hline \multirow{6}{*}{$50 \%$} & 0.3 & 0.545 & 0.206 & 1.000 & 0.000 & 0.997 & .010 & 0.997 & 0.010 & 0.991 & 0.030 \\
\hline & 0.5 & 0.464 & 0.193 & 0.961 & 0.036 & 0.921 & 0.058 & 0.991 & 0.016 & 0.988 & 0.040 \\
\hline & 0.7 & 0.361 & 0.193 & 0.724 & 0.127 & 0.570 & 0.169 & 0.964 & 0.035 & 0.988 & 0.031 \\
\hline & 1.0 & 0.097 & 0.097 & 0.361 & 0.099 & 0.106 & 0.073 & 0.876 & 0.063 & 0.948 & 0.040 \\
\hline & 1.5 & 0.027 & 0.033 & 0.064 & 0.028 & 0.006 & 0.020 & 0.564 & 0.169 & 0.712 & 0.092 \\
\hline & 2.0 & 0.003 & 0.010 & 0.009 & 0.022 & 0.000 & 0.000 & 0.333 & 0.142 & 0.476 & 0.161 \\
\hline
\end{tabular}

